\title{
Correlative Super-Resolution and Atomic Force Microscopy of DNA Nanostructures and Characterization of Addressable Site Defects Supporting Information
}

\author{
Christopher M. Green*, William L. Hughes, Elton Graugnard, and Wan Kuang* \\ *cmgreen011@gmail.com, *wankuang@boisestate.edu
}

\section{Contents}

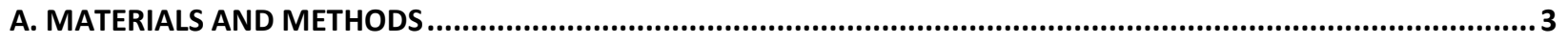

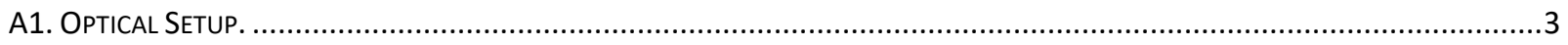

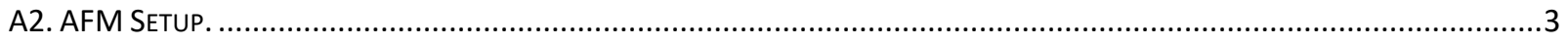

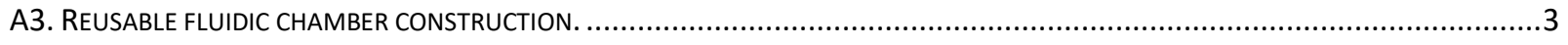

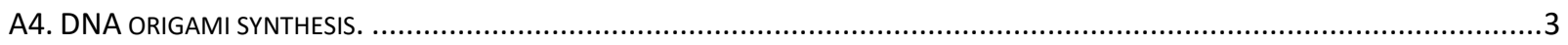

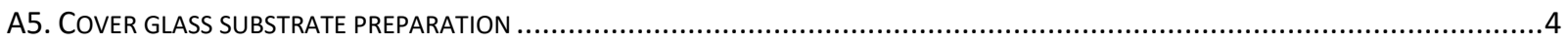

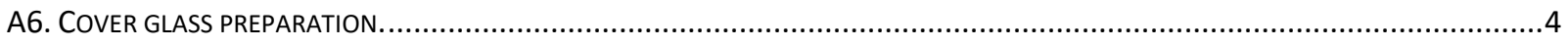

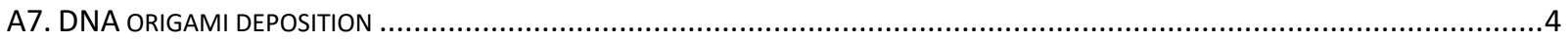

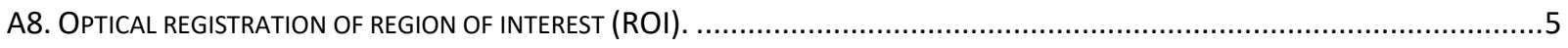

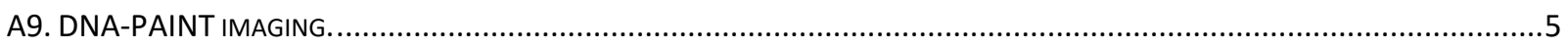

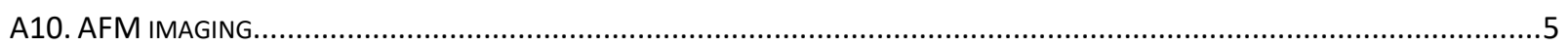

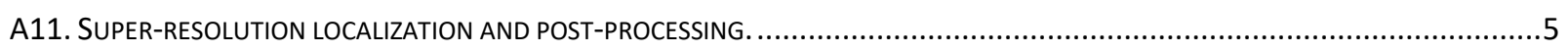

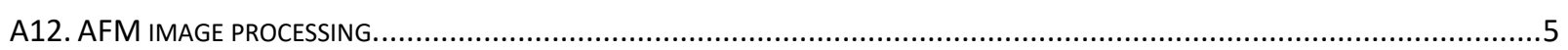

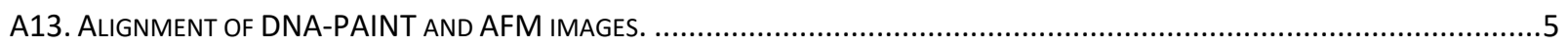

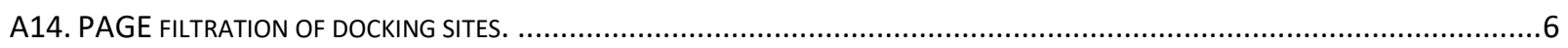

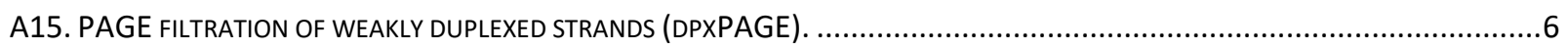

A16. SEQUENCE-TARGED PAGE FILTRATION WITH ACRYDITE-IMMOBILIZED SSDNA (SEQPAGE). ...........................................

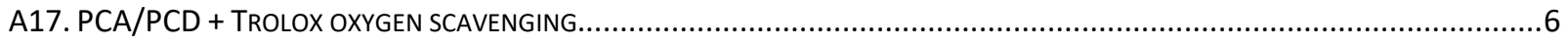

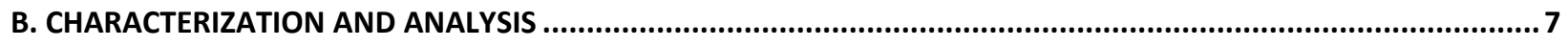

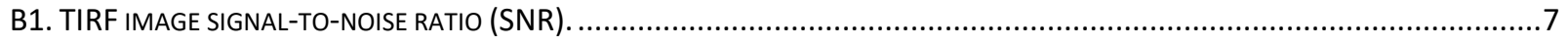

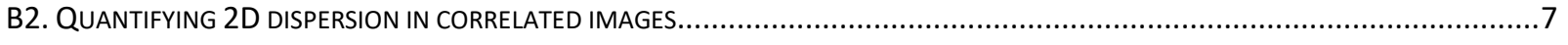

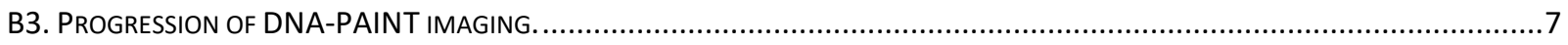

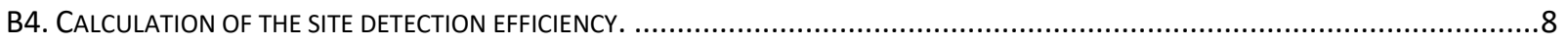

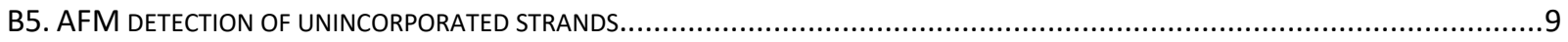

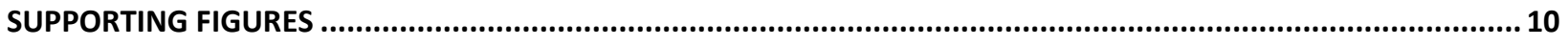

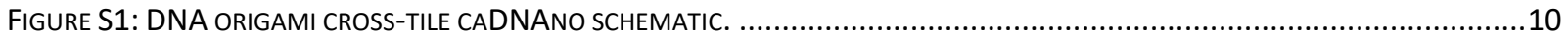


FiguRE S2: SCHEMATIC OF GLOW DISCHARGE VACUUM CHAMBER AND FLUIDIC WELL

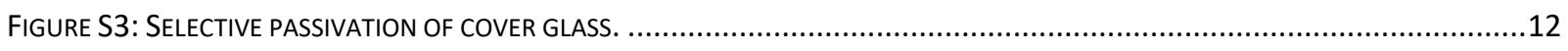

FIGURE S4: COMPARISON OF DNA-PAINT IMAGES ON BSA-PASSIVATED AND PROCESSED COVER GLASS.................................13

FIGURE S5: HIGH RESOLUTION AFM ON PROCESSED COVER GLASS......................................................................... 14

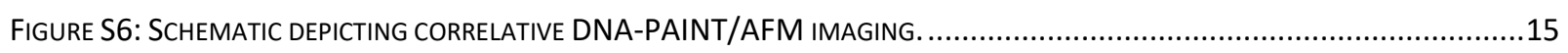

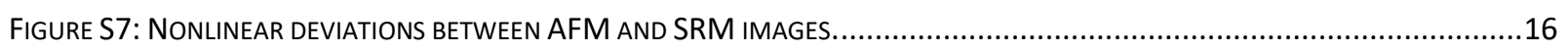

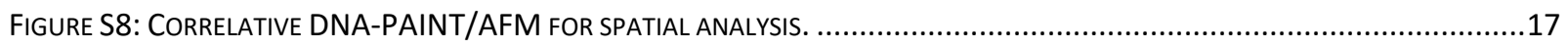

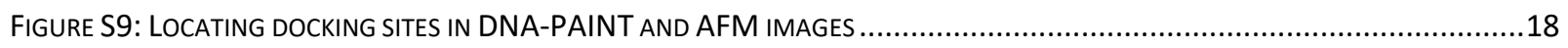

FIGURE S10: CORRELATION OF DOCKING SITE POSITIONS IN AFM AND DNA-PAINT IMAGES ..........................................19

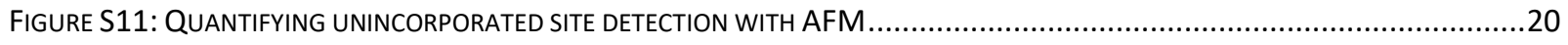

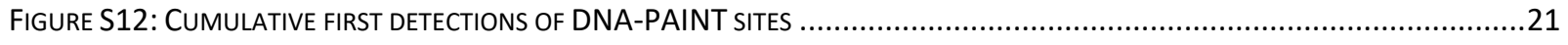

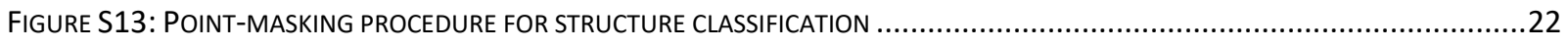

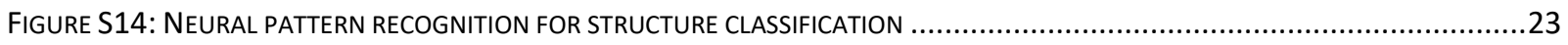

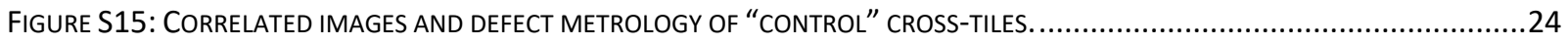

FIGURE S16: CoRRELATED IMAGES AND DEFECT METROLOGY OF “PAGE-FILTERED” CROSS-TILES........................................25

FiguRE S17: CoRRELATED IMAGES AND DEFECT METROLOGY OF “DPXPAGE-FILTERED” CROSS-TILES .......................................26

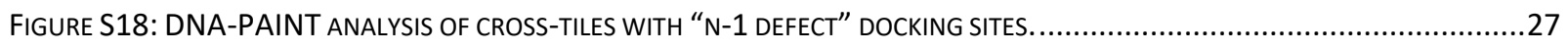

FIGURE S19: DNA-PAINT ANALYSIS OF CROSS-TILES WITH “PAGE-FILTERED, N-1 DEFECT” DOCKING SITES.................................28

FIGURE S20: DNA-PAINT ANALYSIS OF CROSS-TILES WITH “SEQPAGE-FILTERED, N-1 DEFECT” DOCKING SITES ...........................29

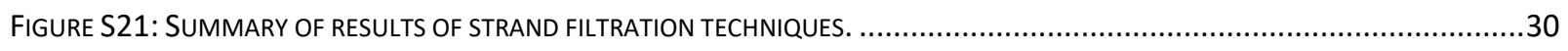

FIGURE S22: DNA-PAINT IMAGES OF DNA ORIGAMI CROSS-TILES WITH VARYING DURATION OF UV EXPOSURE ..........................31

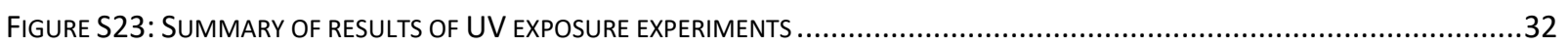

FIGURE S24: CoRRELATED IMAGES AND DEFECT METROLOGY OF “CONTROL” CROSS-TILES.....................................................33

FIGURE S25: CoRRELATED IMAGES AND DEFECT METROLOGY OF “CONTROL” CROSS-TILES WITH OXYGEN SCAVENGERS....................34

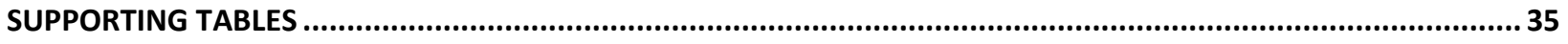

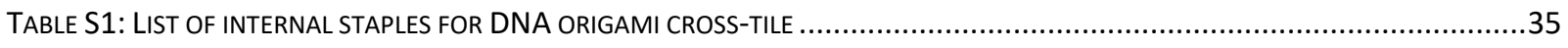

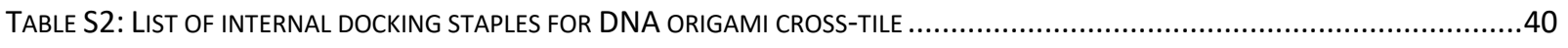

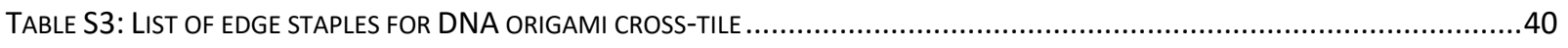

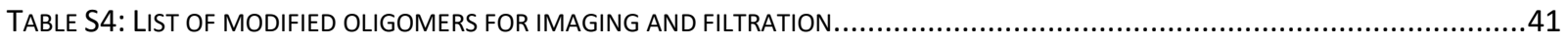

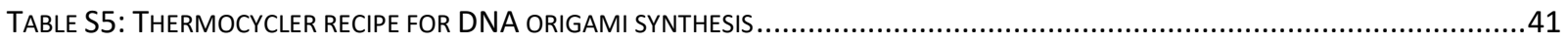

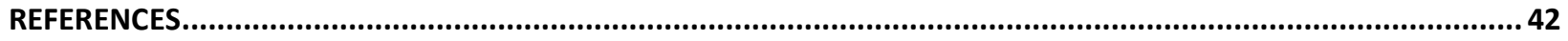




\section{A. Materials and Methods}

Unmodified DNA oligonucleotides (Tables S1-S3) were purchased from Integrated DNA technologies. Cy3b modified DNA oligomers (Table S4) were purchased from Bio-Synthesis. M13mp18 scaffold was purchased from Bayou Biolabs (cat: P-107). Agarose (cat: R0492), 10X Tris-borate EDTA (TBE, $890 \mathrm{mM}$ Tris, $890 \mathrm{mM}$ boric acid, $20 \mathrm{mM}$ EDTA, cat: FERB52), 100X Tris-EDTA (cat: BP1338-1), magnesium chloride $\left(\mathrm{MgCl}_{2}\right)$ hexahydrate (cat: AC197530010), and SYBR ${ }^{\mathrm{TM}}$ gold nucleic acid gel stain (cat: S11494) were purchased from Fisher Scientific. 20\% Ficoll@ solution (cat: F5415-50ML) was purchased from Sigma Aldrich. 30\% Acrylamide/Bis solution 29:1 (cat: 1610156) was purchased from Bio-Rad. Tetramethylethylenediamine (TEMED, cat: BP150-100) and ammonium persulfate (APS, cat: AC327081000) were purchased from Fisher Scientific. Cover glass mounts were designed and machined in house. Gold Seal ${ }^{\circledR} \# 1$ cover glass (cat: 260341) were purchased from Ted Pella. $150 \mathrm{~nm}$ silane polymercoated spherical AuNPs (part: E11-150-Silane-2.5) were custom ordered from Nanopartz. Alconox ${ }^{\mathrm{TM}}$ Liquinox ${ }^{\text {TM }}$ (cat: NC9906065) and methanol (cat: AA19393K2) were purchased from Fisher Scientific. TypeF immersion oil (cat: MOIL-30) was purchased from ThorLabs. Bruker FastScan D AFM tips (cat: FASTSCAN-D) were purchased from Bruker. Fluoroelastomer X-profile o-rings (cat: 6450K126) and 2-56 flathead screws (cat: 92210A076) were purchased from Grainger. Protocatechuic acid (PCA, cat: AAB2401636), protocatechuate-3,4-dioxygenase (PCD, cat: ICN15197525), and 6-hydroxy-2,5,7,8tetramethylchroman-2-carboxylic acid (Trolox, cat: AC218940250) were purchased from Fisher Scientific. Purified and deionized (DI) water was acquired from a Barnstead ${ }^{\mathrm{TM}}$ Nanopure $^{\mathrm{TM}}$ water purification system from Thermo Scientific.

A1. Optical Setup. Optical imaging was performed on a modified Nikon Eclipse TiU microscope equipped with a Nikon Total Internal Reflection Fluorescence (TIRF) illuminator and CFI Apo TIRF 100x NA 1.49 objective. A $561 \mathrm{~nm}$ Coherent Sapphire laser was used for excitation with a 0.5x stop down (approx. $8 \mathrm{~mW}$ TIRF illumination), and spectral filtration was performed with a Chroma TRF49909 ET-561 nm filter set. An additional 1.5x magnification was used to achieve a total magnification of $150 \mathrm{x}$ and pixel size of $107 \mathrm{~nm}$. Images were acquired using a Princeton Instruments ProEM EMCCD camera controlled by the imaging software LightField, set to 100x EM gain with low analog gain and $150 \mathrm{~ms} /$ frame $(6.67 \mathrm{~Hz})$ acquisition. The area captured within each image is $55 \times 55 \mu^{2}$ with a $512 \times 512 \mathrm{px}$ sensor ROI. Focal drift was corrected in real time with an optical setup and feedback loop controlling a Mad City Labs (MCL) Nano-Drive piezo stage. Precise stage movements for registration were performed with an MCL Micro-Drive stepper motor stage.

A2. AFM Setup. Atomic force microscopy (AFM) images were acquired in fluid on a Bruker Dimension FastScan with fluid tapping mode and Bruker FastScan D AFM tips. Sample cover glass were mounted on sticky silicone pads for AFM imaging. Typical scanning parameters were $11 \mu \mathrm{m} / \mathrm{s}$ tip velocity with $1 \mathrm{~nm}^{2}$ pixel size for image areas up to $2 \times 2 \mu \mathrm{m}^{2}$, and $40 \mu \mathrm{m} / \mathrm{s}$ tip velocity with $2000 \times 2000 \mathrm{px}$ images acquired for areas up to $20 \times 20 \mu \mathrm{m}^{2}$.

A3. Reusable fluidic chamber construction. For recovery of the substrate after TIRF imaging, reusable cover glass mounts were designed to enable construction and deconstruction of the fluidic chamber without damaging the cover glass substrate. The cover glass mounts (Figure S2b) were machined out of aluminum, and $x$-shaped o-rings were used to seal the open-backed fluidic chamber upon assembly. With this design, the cover glass is held in place by compression against the o-ring and can be easily removed from the mount after TIRF imaging.

A4. DNA origami synthesis. Cross-shaped DNA origami tiles (Figure S1, Tables S1-S3) were prepared with $10 \mathrm{nM} \mathrm{M13mp18}$ scaffold, $50 \mathrm{nM}$ unmodified oligomers, and $500 \mathrm{nM}$ docking oligomers in $0.5 \mathrm{X}$ TBE buffer (44.5 mM Tris, $44.5 \mathrm{mM}$ boric acid, $1 \mathrm{mM}$ EDTA, pH 8.3) with $12.5 \mathrm{mM} \mathrm{MgCl}_{2}$. Thermal annealing was performed in an Eppendorf Mastercycler Nexus Gradient thermal cycler using a previously reported recipe, provided in Table S5. After annealing, cross-tiles and sharp triangles were stained with $0.1 \mathrm{X}$ SYBR $^{\text {TM }}$ Gold and mixed with loading buffer (0.5X TBE, 20\% Ficoll@ solution in water) at 5:1 origami 
solution to loading buffer. DNA origami were filtered by agarose gel electrophoresis $(0.8 \%$ agarose, $0.5 \mathrm{X}$ TBE, $8 \mathrm{mM} \mathrm{MgCl} 2$ ) at $7 \mathrm{~V} / \mathrm{cm}$ for 90 minutes uncooled. Filtered DNA origami structure bands were identified under $305 \mathrm{~nm}$ UV light illumination and cut from the gel, and origami were extracted from the gel by compressing the agarose between glass slides. DNA origami concentrations were determined using a Thermo Scientific ${ }^{\mathrm{TM}}$ NanoDrop $^{\mathrm{TM}}$ One microvolume UV-Vis spectrophotometer.

A5. Cover glass substrate preparation. Prior to cleaning, No. 1 cover glass ( $22 \mathrm{~mm} \times 22 \mathrm{~mm}$ ) were lightly inscribed with a fine-tip diamond scribe in the upper corner of each slide for orientation of the cover glass, and cross-marks were inscribed at the center of each slide to enable registration during imaging. Cover glass were then submerged in DI water with $0.1 \%$ Liquinox $^{\mathrm{TM}}$ surfactant and cleaned by ultrasonic agitation (sonication) for $1 \mathrm{~min}$ to remove contaminants. Following sonication, cover glass were removed from the surfactant solution, submerged in DI water several times, then sonicated again for 1 min in DI water. Cover glass were placed in custom mounts and centrifuged at $1000 \mathrm{rcf}$ for $5 \mathrm{~min}$ to remove excess water, then baked in an incubator at $40^{\circ} \mathrm{C}$ for $>30$ min to remove any remaining water.

After cleaning and drying, $50 \mu \mathrm{L}$ of $200 \mathrm{fM}$ silanized AuNPs in methanol were deposited on the cover glass and incubated for $10 \mathrm{~min}$. During incubation, methanol was added as needed to prevent complete evaporation of the solution. Cover glass were rinsed with methanol, then submerged several times in DI water and dried by slowly withdrawing the cover glass from the water bath. Excess water was wicked from the surface with a lab wipe, then the cover glass were placed in the incubation chamber at $40^{\circ} \mathrm{C}$ and stored until use.

A6. Cover glass preparation. To prepare cover glass for sample deposition, substrates were placed with the AuNP-functionalized surface face up in a glow discharge vacuum chamber (Figure S2a), and the chamber was pumped down to 2 Torr. Valves into the chamber were closed to maintain pressure statically, then the glow discharge was activated for 75 seconds. After glow discharge treatment, the chamber was vented slowly, then the cover glass was assembled into a reusable fluidic chamber for DNA origami deposition and imaging.

Note: It is best to minimize the amount of time that treated substrates are exposed to air and/or heat. If covered with buffer at room temperature, the surface should remain functional for $>8$ hours. This is particularly important for DNA-PAINT imaging; after the surface has reverted, imager strands will begin to adsorb to the surface.

All optical imaging steps were performed with identical buffer conditions, referred to as the working buffer (0.5X TBE with $18 \mathrm{mM} \mathrm{MgCl}_{2}, \mathrm{pH}$ 8.3).

A7. DNA origami deposition. After coverslip preparation and assembly of the fluid well, DNA origami solution ( $100 \mu \mathrm{L}$ of DNA origami at $0.1 \mathrm{nM}$ in working buffer) was deposited directly onto the surface (surface area $\sim 1.77 \mathrm{~cm}^{2}$ ), then the fluidic chamber was sealed to prevent evaporation and the sample was incubated at room temperature for 30 minutes. After incubation, the DNA origami solution was wicked from the fluidic chamber, then the surface was rinsed twice with $200 \mu \mathrm{L}$ working buffer. Excess buffer was then added to the sample and covered until use.

Note: The surface concentration of DNA can be controlled by the concentration of DNA origami, the divalent cation concentration, and the incubation time. High DNA concentrations ( $>1 \mathrm{nM}$ for $100 \mu \mathrm{L}$ volume) result in saturation of the surface with origami, and the degree of surface coverage is dependent on the concentration of $\mathrm{MgCl}_{2}$. Below $72 \mathrm{mM} \mathrm{MgCl}_{2}$ only a single layer of origami is observed; aggregation does not occur. Origami bound to the surface are highly stable and adsorption appears to be effectively irreversible in the buffer conditions described (0.5X TBE with $6 \mathrm{mM}$ to $72 \mathrm{mM} \mathrm{MgCl}$ ).

Note: DNA-PAINT can be performed within approximately 8 hrs of preparation, but the best results are achieved immediately after preparation. If DNA-PAINT will not be performed, the sample can be stored significantly longer, though it is suggested to rinse the surface with filtered buffer prior to storage to reduce adsorption of contaminants to the surface. 
A8. Optical registration of region of interest (ROI). After sample preparation, the fluidic chamber was mounted on the microscope. The additional 1.5x magnification was removed to provide a larger field of view to locate the inscribed registration marks. The registration mark was identified in brightfield, and the center of the cross-point was aligned to the center of the sensor ROI. Steps of $70 \mu \mathrm{m}$ were performed in the $\times$ and $\mathrm{Y}$ axes to move the imaging ROI away from the inscribed registration mark, and images were captured for each move and stitched together to provide a full view of the registration mark and desired ROI. Once the desired ROI was found, the $1.5 \mathrm{x}$ magnification was added and illumination was changed to the $561 \mathrm{~nm}$ laser for TIRF imaging.

A9. DNA-PAINT imaging. For DNA-based points accumulation for imaging in nanoscale topography (DNAPAINT) imaging, two imaging and rinsing buffers were prepared. Imaging solution M1' (Table S4) were prepared with $3 \mathrm{nM}$ of Cy3b-labeled imager strands $\mathrm{M} 1$ ' in working buffer. A passivation solution was prepared with M1 ssDNA (complementary to imager strand M1) at $3 \mu \mathrm{M}$ in working buffer, and excess working buffer was prepared for additional rinsing steps. For single-color image acquisition, $400 \mu \mathrm{L}$ of imaging solution M1' was added to the fluidic chamber. 24,000 frames were acquired with $150 \mathrm{~ms} / \mathrm{frame}$ for a total of $1 \mathrm{hr}$ acquisition. After imaging, the fluidic chamber was rinsed twice with $400 \mu \mathrm{L}$ of filtered working buffer, then the sample was transferred for AFM imaging.

A10. AFM imaging. The fluidic chamber was deconstructed to provide access to the substrate, and the cover glass was placed on a silicone pad and mounted on the AFM stage. The sample was rinsed with 300 $\mu \mathrm{L}$ of filtered working buffer, then $300 \mu \mathrm{L}$ of filtered working buffer with $1 \mathrm{mM} \mathrm{NiCl}_{2}$ was deposited on the surface and incubated for $5 \mathrm{~min}$. For imaging, $300 \mu \mathrm{L}$ of filtered working buffer was deposited on the surface. The AFM tip was aligned over the center of the registration mark, then steps of $70 \mu \mathrm{m}$ were performed in the $\mathrm{X}$ and $\mathrm{Y}$ axes to relocate the correct $\mathrm{ROI}$, accounting for any changes in the orientation of the substrate. The sample was then engaged, and high-resolution AFM images ( $1 \mathrm{~nm} / \mathrm{px}$ and $1 \mu \mathrm{m}$ or $2 \mu \mathrm{m}$ per side) were captured. $50 \mu \mathrm{L}$ of DI water was added to the sample every 30 minutes to counteract evaporation. Large area AFM images $(5 \mu \mathrm{m}, 10 \mu \mathrm{m}$, and/or $20 \mu \mathrm{m}$ per side, $2000 \times 2000 \mathrm{px})$ were then acquired, centered on the original ROI.

A11. Super-resolution localization and post-processing. Image localization, fiducial-based xy-drift correction, and image post-processing were performed with the ThunderSTORM plugin for ImageJ, which is available for download at http://zitmen.github.io/thunderstorm/. ${ }^{1,2}$ The images were filtered to remove localizations with uncertainty greater than $15 \mathrm{~nm}$ and exported at $20 x$ magnification $(5.35 \mathrm{~nm} / \mathrm{px})$ for rough alignment to AFM images. Corresponding DNA-PAINT ROI were identified for each AFM image and rendered at the resolution of the AFM image.

A12. AFM image processing. AFM images were processed with Gwyddion (available at http://gwyddion.net/) using a simple three-step leveling and scar removal procedure. ${ }^{3}$ Images were leveled initially by 'mean plane subtraction', then rows were aligned using the 'median' method. Lastly, horizontal stroke scars were removed, and the images were rendered at the captured resolution using a grayscale LUT for correlated images.

A13. Alignment of DNA-PAINT and AFM images. Initial alignment of DNA-PAINT and AFM images was performed by manual alignment of AuNP fiducial markers identified in the DNA-PAINT and large area AFM images. Within the full DNA-PAINT field of view, ROI were identified corresponding to each AFM image, and each ROI was rendered in ThunderSTORM at a resolution matching the defined AFM image. Next, the MATLAB script cpselect (Control Point Selection tool) was used to identify corresponding points of interest (POI) in the images, generate a geometric transformation to minimize global offsets, and transform the AFM image for overlay on the corresponding SRM image; details can be found at https://www.mathworks.com/help/images/ref/cpselect.html. It was found that topographic images up to $5 \times$ $5 \mu \mathrm{m}^{2}$ were well aligned using a projective transformation; for larger images, locally-weighted mean transformations were required on occasion, likely due to stage drift during AFM imaging. The transformation was applied to the DNA-PAINT image and cropped to the boundaries of the corresponding AFM image. 
The corrected DNA-PAINT image was then overlaid on the AFM image using ImageJ. See Figure S7 for reference.

A14. PAGE filtration of docking sites. For native PAGE filtration, a 10\% native PAGE gel (10\% $29: 1$ acrylamide/bis-acrylamide, 0.1\% w/v APS, 0.064\% v/v TEMED, $1 \mathrm{X}$ TBE, $1.5 \mathrm{~mm}$ thickness and $10 \mathrm{~cm}$ length) was prepared and ran for 30 minutes with $1 \mathrm{X}$ TBE buffer. ssDNA samples ( $50 \mu \mathrm{M}$ ssDNA, $1 \mathrm{X}$ TBE, $6 \%$ Ficoll) were loaded onto the gel and ran uncooled for 120 minutes with $150 \mathrm{~V}$ applied voltage. The completed PAGE gel was imaged on a phosphor plate with $254 \mathrm{~nm}$ illumination (Figures S16,19), then the main sample bands were cut from the gel, crushed, and submerged in $150 \mu \mathrm{L}$ of $1 \mathrm{X}$ TE buffer. After 24 hours, the samples were centrifuged for 5 min @ 10,000 rcf, and the supernatant was extracted from each sample.

A15. PAGE filtration of weakly duplexed strands (dpxPAGE). A second method for docking sitetargeted PAGE filtration was demonstrated on docking site staple strands using the formation of weak duplexes at the docking site. Staple strands were mixed 1:1 with duplexing strands (53 nt total length, 46/47 nt poly-thymine with 3' 8/9 nt M1' domain) at $200 \mu \mathrm{M}$ in 1X TBE with $12.5 \mathrm{mM} \mathrm{MgCl}_{2}$ and $6 \%$ Ficoll, and the mixture was heated to $90{ }^{\circ} \mathrm{C}$ and cooled to $20^{\circ} \mathrm{C}$ over 20 minutes. A $10 \%$ native PAGE gel $(10 \% 29: 1$ acrylamide/bis-acrylamide, 0.1\% w/v PSA, 0.064\% w/v TEMED, 1 X TBE, $12.5 \mathrm{mM} \mathrm{MgCl}, 1.5 \mathrm{~mm}$ thickness and $10 \mathrm{~cm}$ length) was prepared, cooled to $5{ }^{\circ} \mathrm{C}$, and ran for 30 minutes at $150 \mathrm{~V}$ with $1 \mathrm{X}$ TBE and 12.5 $\mathrm{mM} \mathrm{MgCl}$. The annealed ssDNA samples were loaded onto the gel and ran for 120 minutes with $150 \mathrm{~V}$ applied voltage, cooled to $5^{\circ} \mathrm{C}$. The completed PAGE gel was imaged on a phosphor plate with $254 \mathrm{~nm}$ illumination (Figure S17), then the main sample bands were cut from the gel, crushed, and submerged in $150 \mu \mathrm{L}$ of $1 \mathrm{X}$ TE buffer. After 24 hours, the samples were centrifuged for 5 min @ 10,000 rcf, and the supernatant was extracted from each sample.

A16. Sequence-targeted PAGE filtration with Acrydite-immobilized ssDNA (seqPAGE). For docking site-targeted PAGE filtration, short Acrydite-labeled ssDNA strands (sequence M1') were added to a 10\% native PAGE gel at $3 \mu \mathrm{M}$ concentration prior to polymerization of the gel (10\% 29:1 acrylamide/bisacrylamide, 0.1\% w/v PSA, 0.064\% w/v TEMED, 1 X TBE, $12.5 \mathrm{mM} \mathrm{MgCl}_{2}, 3 \mu \mathrm{M}$ Acry-5T-M1', $1.5 \mathrm{~mm}$ thickness and $10 \mathrm{~cm}$ length). Acrydite, an acrylamide phosphoramidite, polymerizes with acrylamide upon the addition of the polymerizing agents APS and TEMED, covalently binding Acrydite-labelled ssDNA into the gel. ${ }^{4}$ The immobilized ssDNA was synthesized with a 7 nt domain complementary to M1 docking sites, thus staple strands with an active docking site could transiently hybridize to immobilized ssDNA, slowing progression through the gel. The gel was cooled to $5^{\circ} \mathrm{C}$ and ran for 30 minutes at $150 \mathrm{~V}$ with $1 \mathrm{X}$ TBE and $12.5 \mathrm{mM} \mathrm{MgCl}_{2}$. ssDNA samples (100 $\mu \mathrm{M}$ ssDNA, $1 \mathrm{X}$ TBE, $12.5 \mathrm{mM} \mathrm{MgCl}_{2}, 6 \%$ Ficoll) were loaded onto the gel and ran for 120 minutes with $150 \mathrm{~V}$ applied voltage, cooled to $5^{\circ} \mathrm{C}$. The completed PAGE gel was imaged on a phosphor plate with $254 \mathrm{~nm}$ illumination (Figure S20), then the main sample bands were cut from the gel, crushed, and submerged in $150 \mu \mathrm{L}$ of $1 \mathrm{X}$ TE buffer. After 24 hours, the samples were centrifuged for 5 min @ 10,000 rcf, and the supernatant was extracted from each sample.

A17. PCA/PCD + Trolox oxygen scavenging. Imaging buffer containing the PCA/PCD oxygen scavenging system were prepared with $3 \mathrm{nM}$ of Cy3b-imaging strands, $0.385 \mathrm{mg} / \mathrm{mL}$ of PCA, $6.99 \mu \mathrm{g} / \mathrm{mL}$ of PCD, and $252 \mathrm{ng} / \mathrm{mL}$ of Trolox in $10 \mathrm{mM}$ Tris-HCl and $1 \mathrm{mM}$ EDTA (protocol adapted from Blumhardt et al.). ${ }^{5}$ Imaging buffer was prepared 1 hour prior to imaging. Imaging was performed in a microchannel constructed on a standard microscope slide with double-sided tape and cover glass, sealed with highvacuum grease to prevent oxygen from diffusing into the imaging buffer during DNA-PAINT. After DNAPAINT, a magnetic puck was attached to the outward facing side of the cover glass with epoxy, and a diamond-tipped scribe was used to score the cover glass around the puck. A strong magnet was used to break the cover glass along the outer scoring, removing the imaged sample area for AFM characterization. 


\section{B. Characterization and Analysis}

B1. TIRF image signal-to-noise ratio (SNR). The SNR of fluorescent events in TIRF images was determined from parameters of the fitted symmetric $2 \mathrm{D}$ gaussian point spread function $\left(P S F_{G}\right)$ for individual events:

$$
P S F_{G}(x, y, i)=\frac{I_{i}}{2 \pi \sigma_{i}^{2}} \exp \left(-\frac{\left(x-X_{i}\right)^{2}+\left(y-Y_{i}\right)^{2}}{2 \sigma_{i}^{2}}\right)+B_{i}
$$

where $I_{i}$ is the total intensity of event $\mathrm{i}, \sigma_{i}$ is the spread, $X_{i}$ and $Y_{i}$ are the localized sub-pixel coordinates of point source $\mathrm{i}$, and $B_{i}$ is the local background intensity offset. The SNR was calculated with $I_{i}$ as the signal and $B_{i}$ as the noise for individual events:

$$
S N R=\sqrt{\frac{\sum I_{i}{ }^{2}}{\sum B_{i}{ }^{2}}}
$$

B2. Quantifying 2D dispersion in correlated images. To quantify the 2D dispersion of correlated points in DNA-PAINT and AFM images, the MATLAB cpselect tool was adapted for manual identification of positions of corresponding POI in the corrected DNA-PAINT image and the original AFM image. 286 corresponding POI were identified in two sets of correlated images with $1 \mathrm{~nm} / \mathrm{px}$ image resolution. The spatial deviation of each DNA-PAINT POI from the corresponding AFM POI was calculated, and the 2D dispersion was calculated as the standard deviation of DNA-PAINT sites from AFM,

$$
\sigma_{x y}=\sqrt{\frac{1}{n-1} \sum\left(\left(X_{i}-x_{i}\right)^{2}+\left(Y_{i}-y_{i}\right)^{2}\right)}
$$

where $X_{i}, Y_{i}$ are the $\mathrm{x} / \mathrm{y}$ coordinates of site $\mathrm{i}$ in the AFM image, $x_{i}, y_{i}$ are the $\mathrm{x} / \mathrm{y}$ coordinates of site $\mathrm{i}$ in the DNA-PAINT image, and $n$ is the total number of sites examined. For the sites examined, $\sigma_{x y}=5 \pm 3 \mathrm{~nm}$. The offsets were also plotted as a function of position to ensure uniform alignment across correlated images. The results are shown in Figure 3 and S10.

B3. Progression of DNA-PAINT imaging. For DNA-PAINT, site detections occur stochastically during image acquisition as fluorophore-labeled imager strands transiently bind to docking sites on the DNA origami. As a result, image acquisition must be performed over a duration that reliably enables image acquisition to surpass a desired degree of completion, occurring when nearly all active docking sites have interacted with imager strands. To ensure complete image acquisition for correlative defect analysis, the progress of imaging was quantified by approximating the fraction of active sites resolved in the final rendered image.

To calculate the fraction of active sites detected during DNA-PAINT imaging, a full field view of DNA-PAINT image was rendered at $5.35 \mathrm{~nm} /$ pixel. Maxima were identified in the rendered image with an intensity threshold of two localizations. The list of positions of maxima within the image was exported. To calculate the number of localizations per POI as a function of time, a MATLAB script was used to perform a search of the full localizations list to identify localizations corresponding to individual POI. Each POI was assigned a search radius $R$ as a function of the average localization uncertainty $\bar{\sigma}_{l o c}$ for all events in the localization list, $R=2 \bar{\sigma}_{l o c}$. A time-resolved POI list was generated containing the position of each POI and a count of the cumulative localizations per minute for each POI. The cumulative number of first detections of POI (i.e., the total number of unique docking sites detected) in time was calculated from the time-resolved list. The cumulative first detections, $N(t)$, can be approximated with a two-part exponential decay,

$$
N(t)=A_{1}\left(1-\exp \left(-k_{1} t\right)\right)+A_{2}\left(1-\exp \left(-k_{2} t\right)\right)
$$


where $k_{1}$ and $k_{2}$ are the detection rates for two independent populations of reaction sites with binding rates $k_{1}$ and $k_{2}$, assuming pseudo-first order kinetics. ${ }^{6}$ For DNA origami cross-tiles imaged with the standard imaging buffer, it was found that the secondary population of reaction sites was large $\left(A_{2}>>A_{1}\right)$ with a low rate constant $\left(k_{2}<<k_{1}\right)$. Given that the number of active POI is fixed during imaging, the secondary population was speculated to be the result of nonspecific imager strand interactions with the substrate. For $t<1 / k_{2}$, the cumulative first detections associated with the second population of sites can be approximated to be linear with respect to time,

$$
\text { for } t \ll \frac{1}{k_{2}}, \quad A_{2}\left(1-\exp \left(-k_{2} t\right)\right) \cong A_{2} k_{2} t .
$$

The cumulative first detections can thus be approximated,

$$
N(t)=N_{\text {total }}\left(1-\exp \left(-k_{1} t\right)\right)+c t,
$$

where $N_{\text {total }}$ and $k_{1}$ are the total number of active sites and the reaction rate constant, respectively, for DNAPAINT, and $\mathrm{c}$ is the correction factor for nonspecific events, approximated as $\mathrm{c}=A_{2} k_{2}$. The fraction of active sites detected, $\theta(\mathrm{t})$, as a function of time for DNA-PAINT was thus approximated as

$$
\theta(t)=\frac{N(t)-c t}{N_{\text {total }}}=1-\exp \left(-k_{1} t\right)
$$

For comparison, the reaction rate constant can also be approximated from the total number of detections $\left(D_{\text {total }}\right)$, the number of unique POI identified $\left(N_{\text {total }}\right)$, and the dissociation rate constant $\left(k_{\text {off }}\right)$ assuming the system has reached thermodynamic equilibrium, indicated by a constant flux of imager strands. The rate equation can then be expressed,

$$
\frac{d I}{d t}=k_{o n}[I][P]-k_{o f f}[I P]=0
$$

where $\left[I_{e}\right],\left[P_{e}\right]$, and $\left[I P_{e}\right]$ are the equilibrium concentrations of imager strands, active $\mathrm{POI}$, and bound imager strands, respectively. For active sites $P$ and bound imager strands $I P$ confined to a surface, $[P]$ and $[I P]$ were approximated from the experimentally determined $N_{\text {total }}$ and the average detections per frame for the case where $\left[P_{e}\right]>>\left[I P_{e}\right]$,

$$
\left[P_{e}\right]=\frac{N_{\text {total }}}{\text { Area }}-\left[I P_{e}\right] \approx \frac{N_{\text {total }}}{\text { Area }}
$$

and

$$
\left[I P_{e}\right] \approx \frac{D_{\text {total }}}{\text { Area }} * \frac{d t}{t_{\text {total }}}=\frac{\bar{D}}{\text { Area }}
$$

and the reaction rate constant, $k_{1}$, for DNA-PAINT was calculated,

$$
k_{1}=k_{o n} *\left[I_{e}\right]=k_{o f f} \frac{\left[I P_{e}\right]}{\left[P_{e}\right]}=k_{o f f} \frac{\bar{D}}{N_{\text {total }}} .
$$

The reaction rate constant and the total imaging time can then be used to approximate the fraction of active sites detected during DNA-PAINT imaging with eq. 7. For the DNA origami cross-tile sample analyzed in Figure S12, the reaction rate constant $k_{1}=0.00273 \mathrm{~s}^{-1}$, in close agreement with the value calculated from the cumulative first detections, $k_{1}=0.00263 \mathrm{~s}^{-1}$.

B4. Calculation of the site detection efficiency. To calculate the site detection efficiency for each DNAPAINT sample, DNA-PAINT images were rendered at 20x with ThunderSTORM, and maxima were identified with a threshold of two localization events, generating a binary image of maxima. To reduce the probability of incorrect counts caused by overlapping structures, points in the binary image were dilated to 
$50 \mathrm{~nm}$, causing structures to overlap if a structure is located within $100 \mathrm{~nm}$ of another structure. To remove overlapping structures, the dilated structures were filtered by total area, and structures with area greater than expected for an individual structure were filtered out of the image (Figure S13). The center of mass was calculated for the remaining structures, then a list of the center of masses was exported from ImageJ. A MATLAB script was used to identify individual structures in the maxima image using the center of mass list and generate input vectors for classification by neural pattern recognition (NPR) using the MATLAB nprtool (Figure S14); details can be found at https://www.mathworks.com/help/deeplearning/gs/classifypatterns-with-a-neural-network.html.

To train the network, SRM images of DNA origami cross-tiles were examined manually to identify tiles with two, three, and four resolved docking sites, as well as overlapping structures with greater than four sites resolved. A minimum of 500 training images were identified for each case, and additional training images were generated by rotational and mirror transformations of the manually selected training images. To reduce the size of the input vector for each structure and improve the performance of NPR, unique input vectors consisting of 53 elements were constructed for each structure. The input vectors were generated by concatenating the radial distribution function of each image with the count of the total number of sites detected. The NPR tool was used to generate a neural network with 10 hidden neurons, and the network was trained to classify tiles by the number of sites resolved using over 4000 training images for each class. Performance testing during training found less than $1 \%$ error for classified structures.

Structures were classified by the number of sites resolved, and structures with uncertain classifications or less than two sites resolved were discarded as they cannot be distinguished from non-specific binding of imager strands to the substrate. The remaining distribution of classified structures was fitted with a binomial probability distribution function,

$$
f(x \mid 4, p)=N\left(\begin{array}{l}
4 \\
x
\end{array}\right) p_{\text {det }}^{x}\left(1-p_{\text {det }}\right)^{4-x}
$$

for $2 \leq x \leq 4$ to calculate the probability of detection $\left(p_{\text {det }}\right)$ of individual sites for each sample. To ensure consistent performance of the automated structure classification, 5 images, each containing 500 structures, were classified manually, and the results were compared to automated classification. For the five images, the maximum deviation in $p_{d e t}$ between manual and automated classification was less than $1 \%$ (Figure S14).

After training the network, classification could be performed on all qualifying structures in a DNA-PAINT image (typically several thousand structures) in a few seconds. Manual classification of the same structures would be impractical. With this method, the detection efficiency could be calculated as a function of time, and the progression shown in Figure S12c was determined from classification of over 60,000 structures. Automated classification was also used to determine the detection efficiencies reported in Figures S15-25.

B5. AFM detection of unincorporated strands. To ensure that unincorporated sites could be reliably detected in topographic AFM images, we performed a series of imaging optimization experiments on DNA origami designed to have both incorporated and unincorporated sites. To quantify the detection of unincorporated site with AFM, DNA origami cross-tiles were synthesized with unincorporated sites by intentionally excluding strands P-L6 and P-R1 (Table S4) during synthesis. All docking sites were included in the synthesis; the excluded strands, located on two opposite corners of tile arm LR, served solely as passivation against blunt-end stacking interactions between tiles. Following synthesis, cross-tiles were filtered with agarose gel electrophoresis (section A4). Samples were prepared for correlative DNAPAINT/AFM microscopy and imaged according to the protocols described in sections A5 to A13. During AFM imaging, two images were captured (Figure S11) at $1 \mathrm{~nm}^{2}$ pixel size and $4 \mathrm{~nm}^{2}$ pixel size, respectively, to determine the imaging resolution necessary to detect unincorporated sites.

For defect analyses, edge detection methods were utilized in Gwyddion to enhance the visibility of features on tile edges. Cross-tiles within the AFM images were examined for edge defects, indicated by a shortened tile edge which appears as an indentation in the corner of a tile arm or rounding of the corner. Within the 
images shown in Figure S11a,b, all intentional unincorporated sites were detected and are indicated by white circles. The edge lengths of unincorporated sites were measured and compared to tile edges without apparent defects, and the results are provided in Figure S11e,f. The observed variation in edge length was $6 \pm 3 \mathrm{~nm}$, in agreement with the value expected for a difference of $16 \mathrm{nt}$ in dsDNA (5.4 nm at $0.34 \mathrm{~nm} / \mathrm{bp})$. The results indicate that unincorporated sites can be reliably detected with AFM under similar conditions.

\section{Supporting Figures}

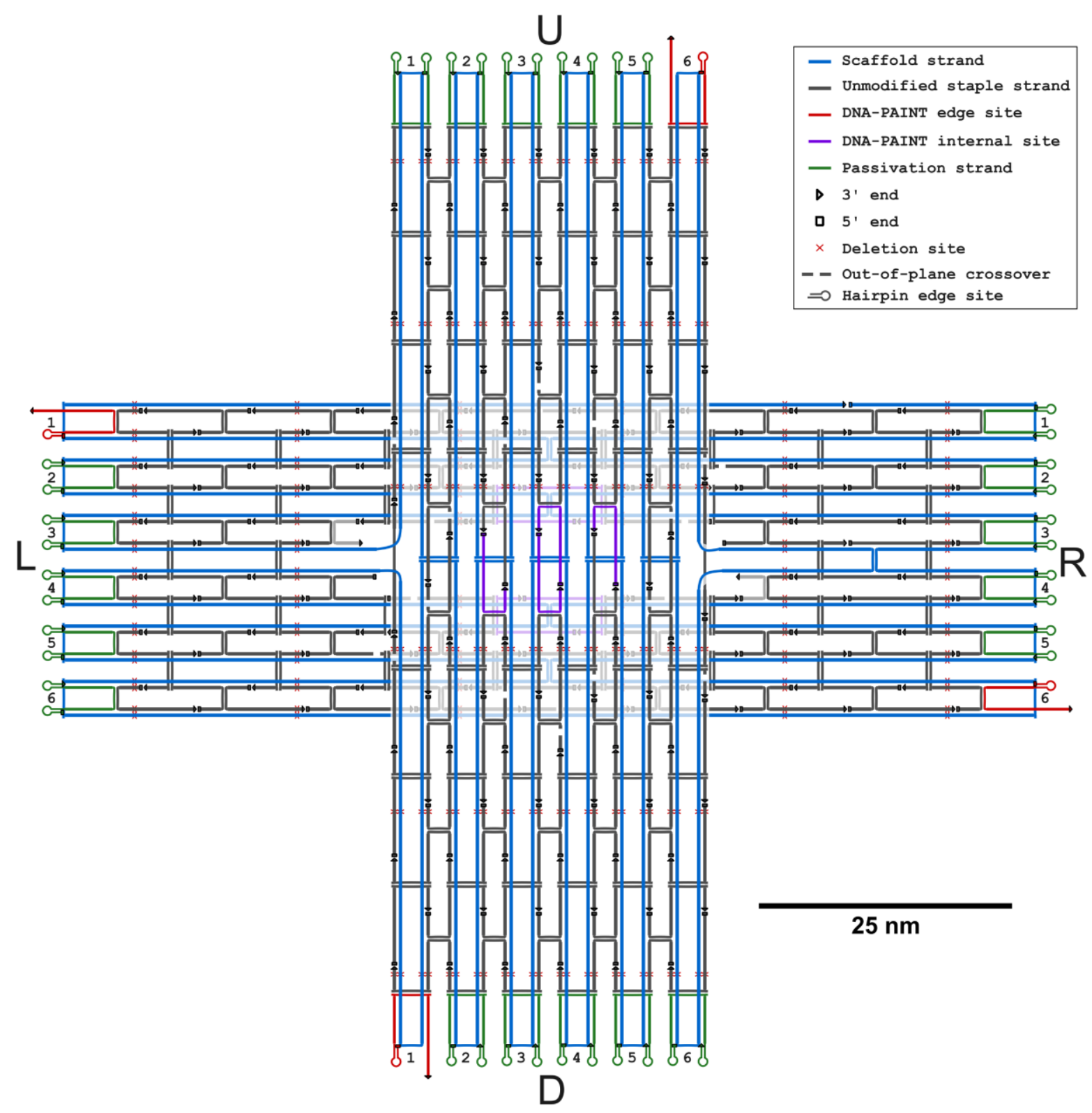

Figure S1: DNA origami cross-tile caDNAno schematic. ${ }^{7}$ Modified caDNAno schematic of the twistcorrected DNA origami cross-tile, ${ }^{8,9}$ altered to more closely depict the geometry of the intended structure. Individual staple strand sequences can be found in Tables S1-S3. Hairpin passivation strands were adapted from Tikhomirov et al. ${ }^{10}$ 


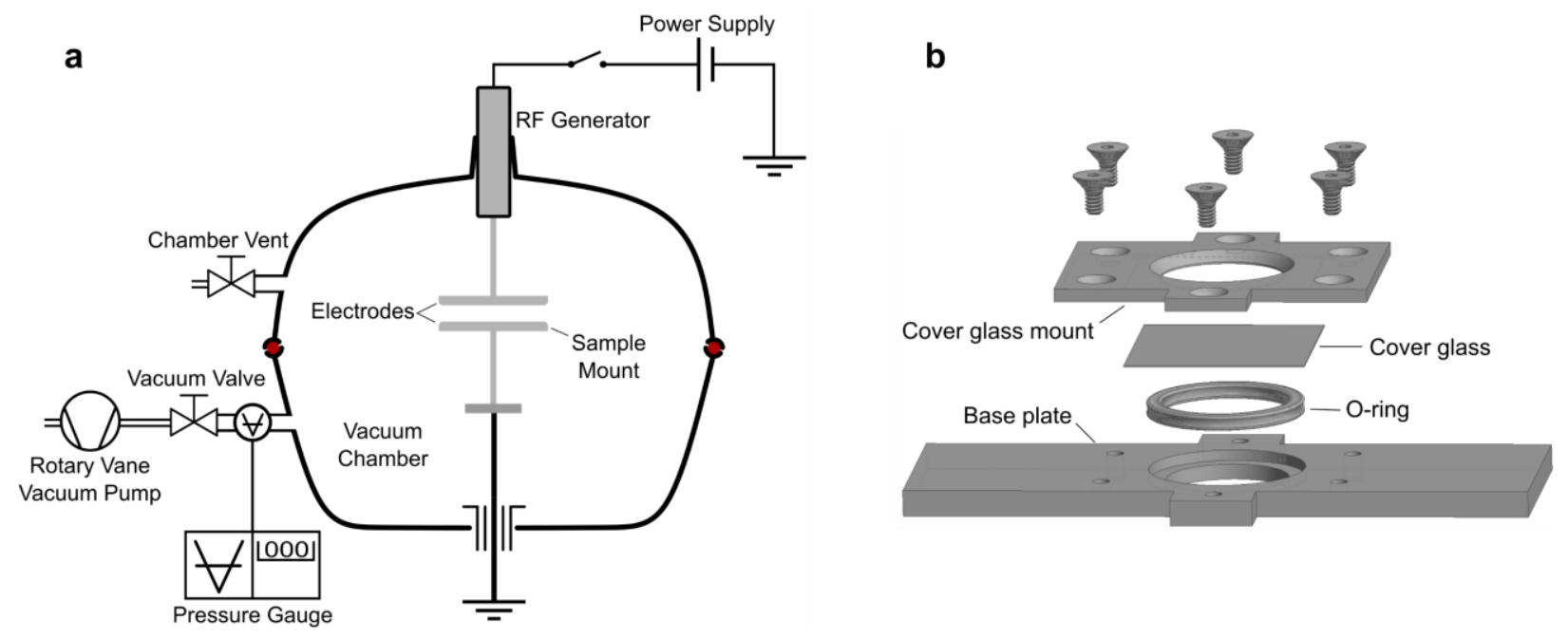

Figure S2: Schematic of glow discharge vacuum chamber and fluidic well. (a) Schematic diagram of the custom glow discharge vacuum chamber used for processing of cover glass. ${ }^{11}$ For glow discharge treatment, power to the RF (radio frequency) generator was supplied for 75 seconds, during which the chamber pressure was held at 2 Torr. (b) 3D CAD model of custom, reusable fluidic well, designed and machined in house. The base plate and cover glass mount were machined from aluminum, and the screws, o-ring, and cover glass were purchased separately. 
a

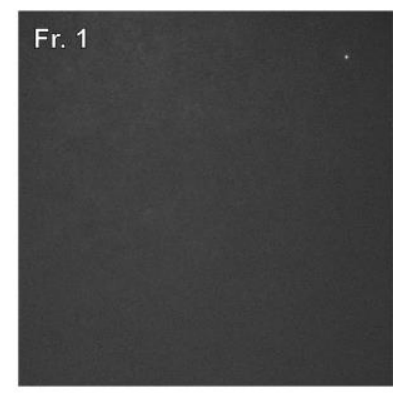

Fr. 3
No DNA origami

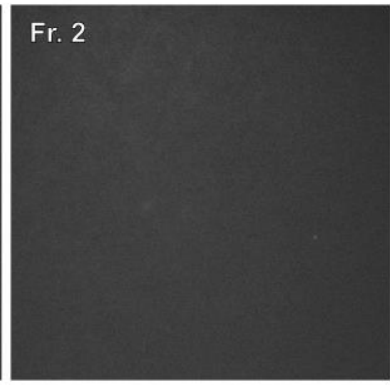

Fr. 4

10 um

b
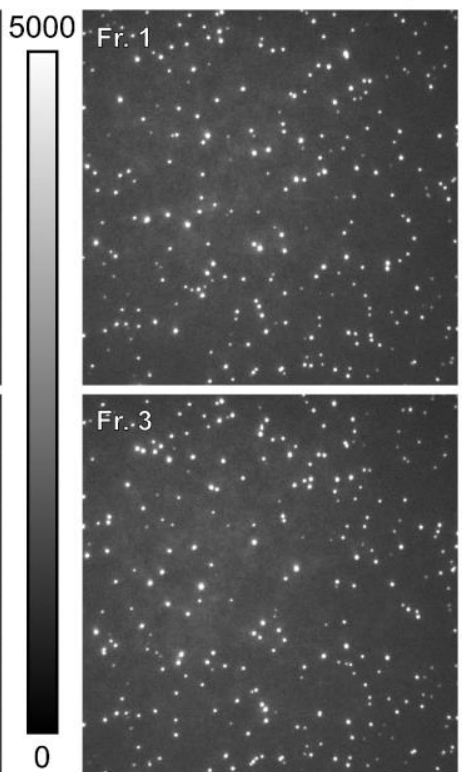
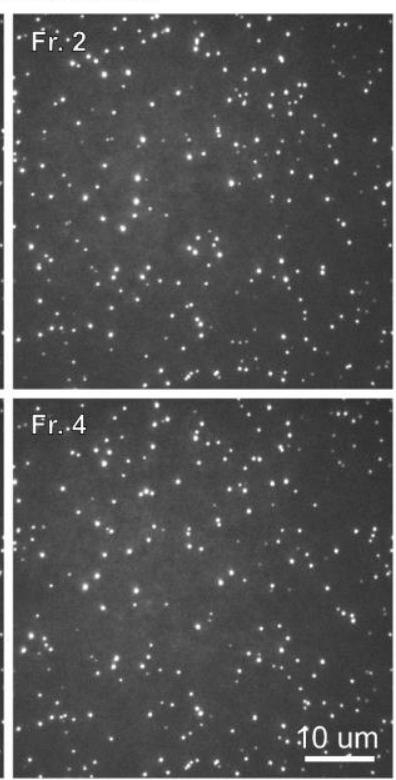

Figure S3: Selective passivation of cover glass. The first four TIRF frames of image acquisition of processed cover glass (a) without DNA origami and (b) with DNA origami cross-tiles adsorbed onto the surface. The imaging buffer for both samples was $0.5 \mathrm{X}$ TBE with $18 \mathrm{mM} \mathrm{MgCl}_{2}, \mathrm{pH} 8.3$, and $3 \mathrm{nM}$ Cy3blabeled imager strands. The samples were imaged under identical experimental conditions, and the colormap is identical for all images. 

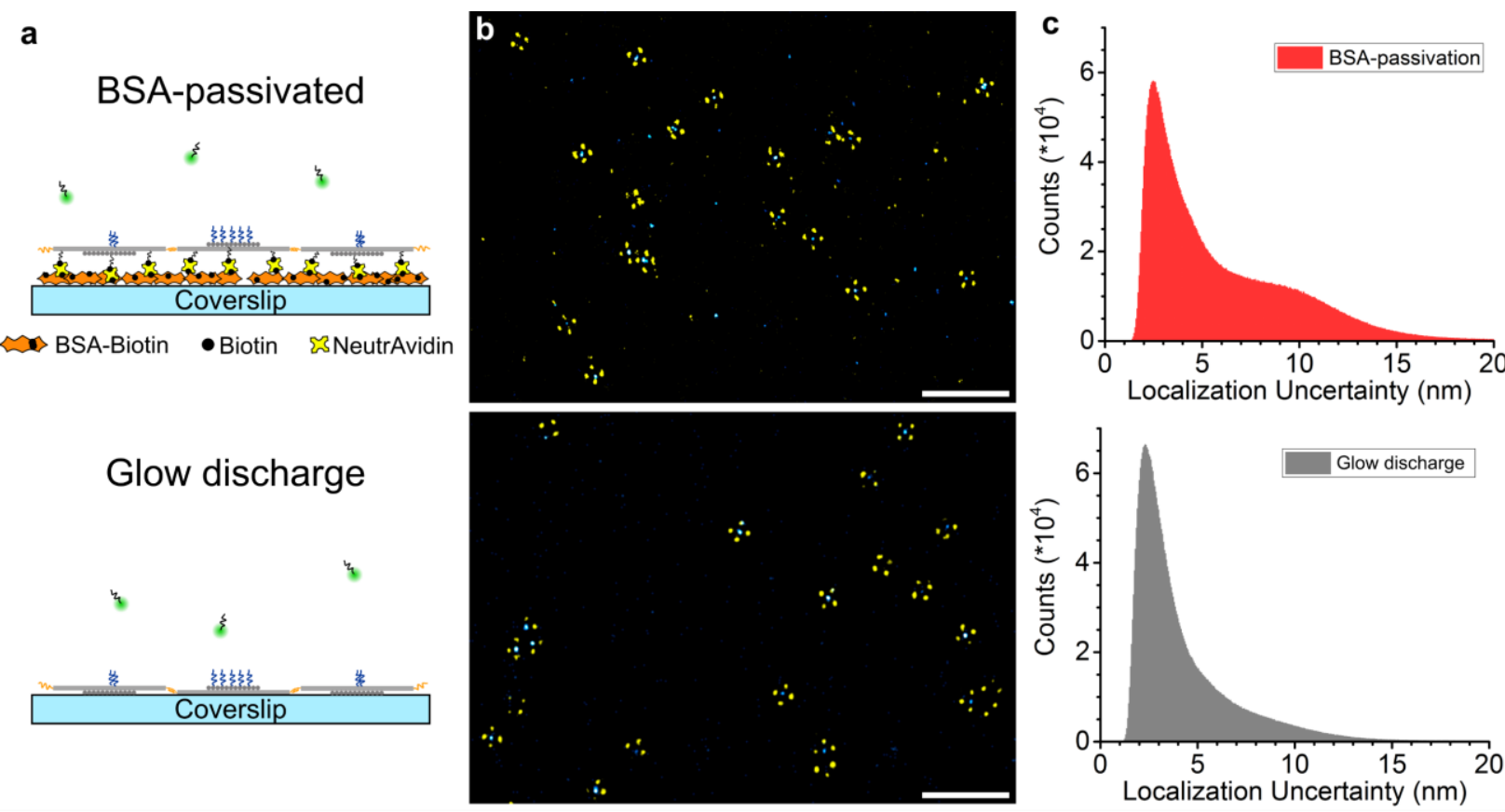

Figure S4: Comparison of DNA-PAINT images on BSA-passivated and processed cover glass. (a) Depiction of DNA origami cross-tiles bound to cover glass by protein-binding (top) and by glow discharge processing (bottom), with (b) multiplexed DNA-PAINT imaging and (c) localization uncertainty distributions shown for each, respectively. The localization lists corresponding to the images in (b) were corrected for $\mathrm{xy}$-drift and rendered at $5.35 \mathrm{~nm} / \mathrm{px}$; no filters were applied to the list prior to image rendering. Nonspecific binding events are indicated in DNA-PAINT images by localizations randomly distributed between structures and in the localization uncertainty distribution by a second population of events with higher localization uncertainty. Scale bars, $500 \mathrm{~nm}$. 


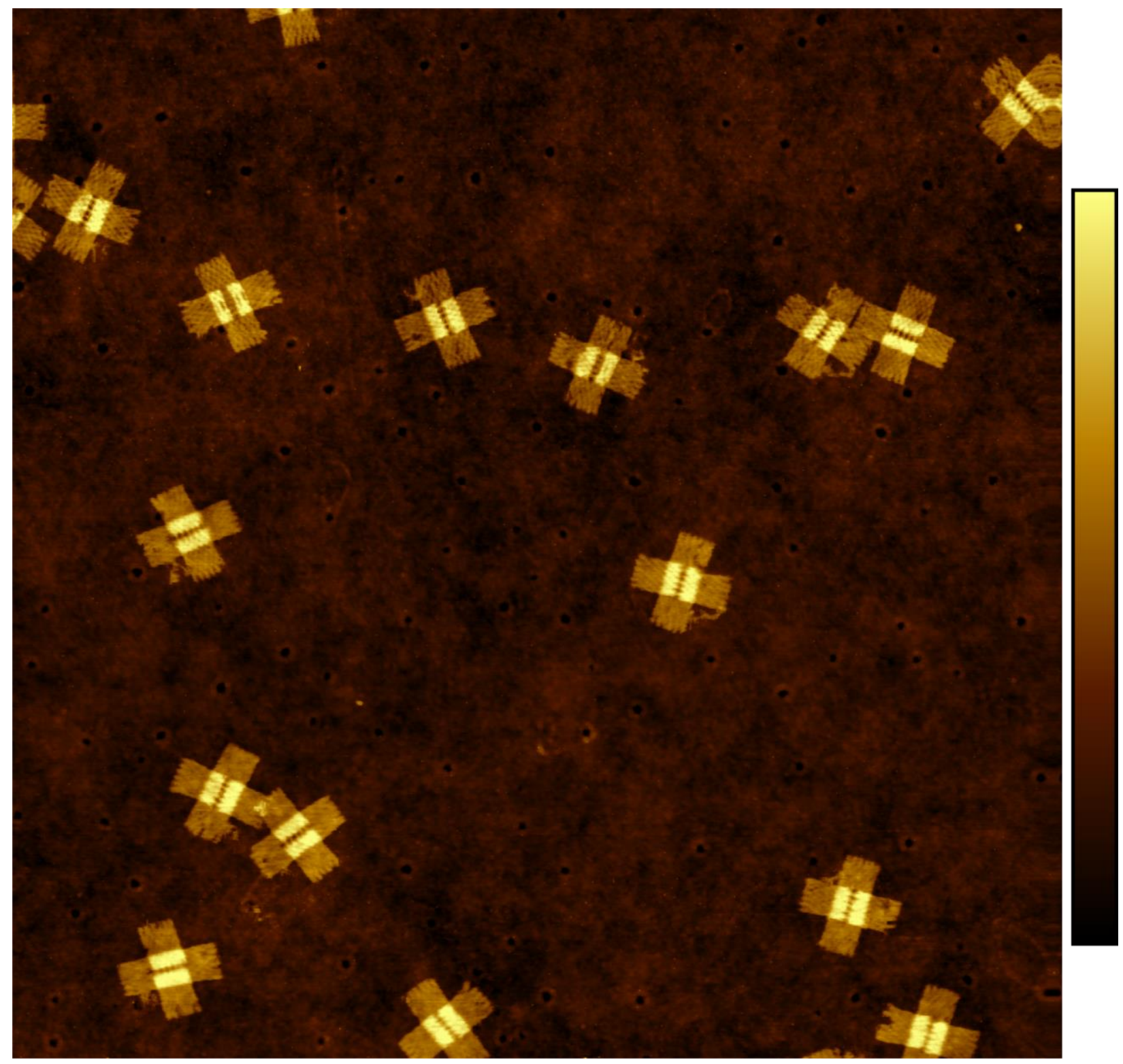

Figure S5: High resolution AFM on processed cover glass. Tapping mode topography images of the DNA origami cross-tile on glow discharge processed cover glass were acquired in fluid (0.5x TBE, $18 \mathrm{mM}$ $\mathrm{MgCl}_{2}$ ). The image shown is $1 \mu \mathrm{m} \times 1 \mu \mathrm{m}$ and was captured with $1000 \mathrm{pts} /$ line and $3 \mathrm{~Hz}$ scan rate. AFM height colormap, $4.2 \mathrm{~nm}$. 


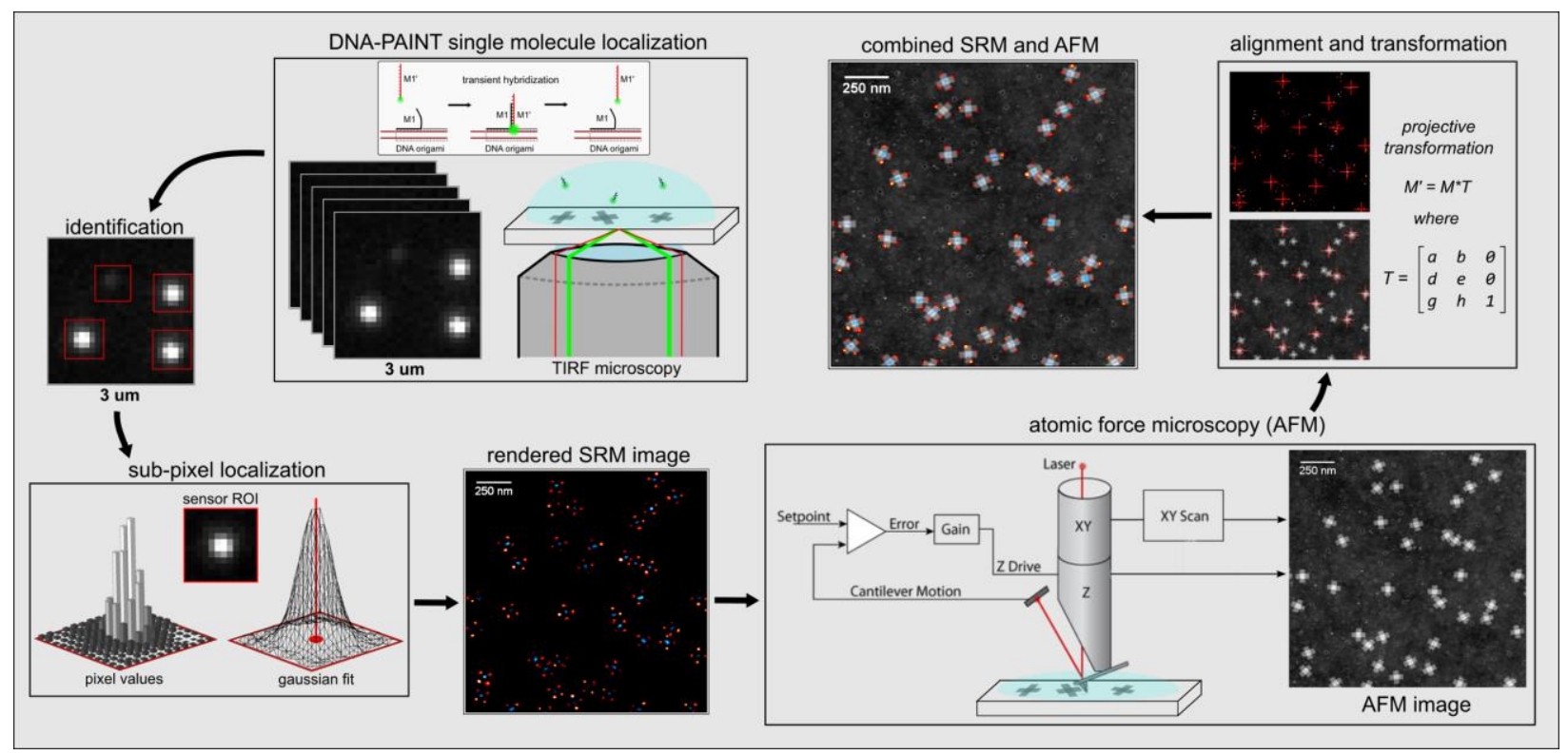

Figure S6: Schematic depicting correlative DNA-PAINT/AFM imaging. For correlative imaging, samples were mounted on an inverted microscope and viewed in brightfield to locate substrate registration marks and perform a programmed stage movement to a specified ROI. DNA-PAINT images of origami within the ROI were then acquired in TIRF illumination by a $561-\mathrm{nm}$ laser source. Fluorescent events were identified in each frame and localized to sub-pixel coordinates, then the positions of each event were corrected for $x y$-drift by tracking fiducial markers as a function of time and generating a translational correction for events in each frame. Super-resolution microscopy (SRM) images were rendered from the corrected localization list, and pseudo-colors were assigned to each image to indicate the imager strand used. After SRM imaging, substrates were removed from the fluidic chamber and transferred for AFM characterization in fluid. The substrate was viewed optically to identify registration marks and move to the ROI imaged with SRM. Topographic images were acquired in fluid tapping mode using protocol provided in the methods. After AFM imaging and image processing, SRM and AFM images were roughly aligned using corresponding structures in the images. The positions of corresponding docking sites in each image were used to generate a projective transformation to transform SRM to AFM and correct for global image aberrations (Figure S7). The corrected SRM and AFM images were then combined by averaging. 

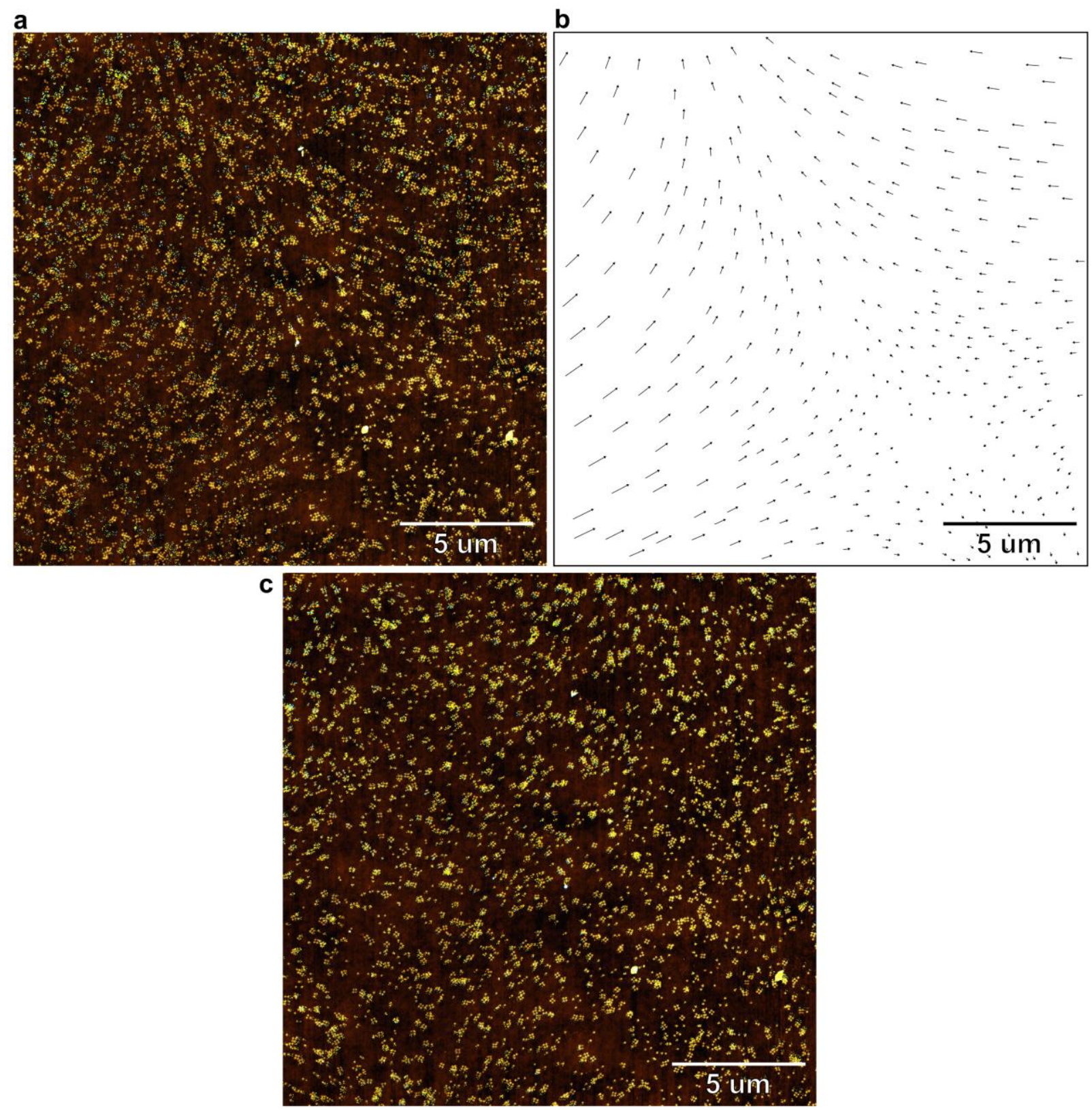

Figure S7: Nonlinear deviations between AFM and SRM images. Global image aberrations often occur in optical and topographic images due to optical aberrations and suboptimal AFM imaging conditions. The aberrations manifest as nonlinear deviations between images acquired on different systems and should be corrected to better represent the correlation between images. (a) Correlative SRM/AFM image of DNA origami cross-tiles with nonlinear deviation in the positions of individual structures within the images. (b) Vector field visualizing the deviation in position of a subset of structures within the image; vectors begin on the AFM structure and end on the SRM structure. (c) Correlative SRM/AFM image from (a) after correcting for global image aberrations. Deviations between individual structures in the image were small and randomly oriented after the correction. 


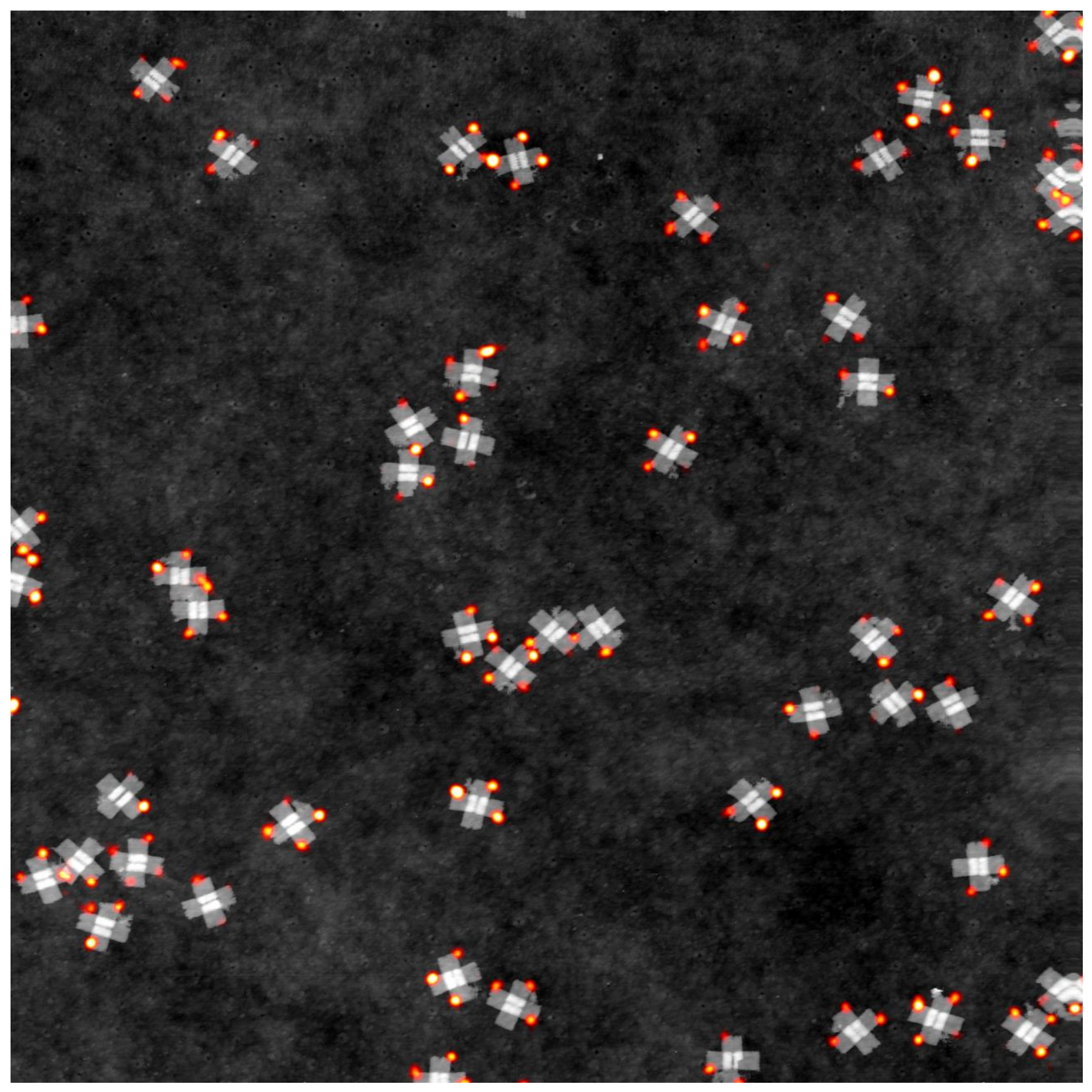

Figure S8: Correlative DNA-PAINT/AFM for spatial analysis. High-resolution, correlative SRM/AFM image of DNA origami cross-tiles on cover glass for quantifying spatial correlation and single-site defect metrology. The AFM image was captured with $1 \mathrm{~nm}^{2}$ pixel size and rendered in greyscale. Image is $2 \times 2$ $\mu m^{2}$. 

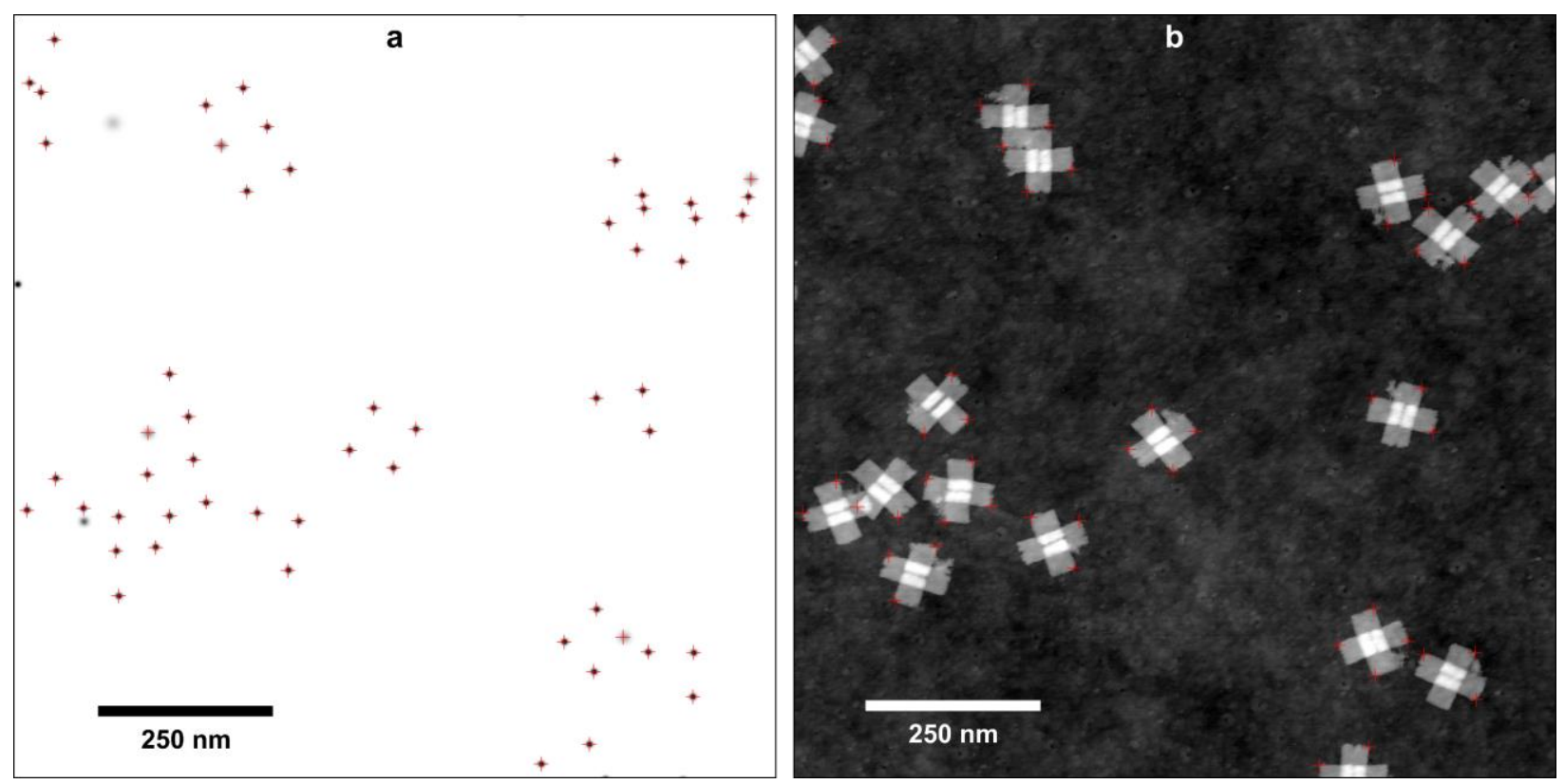

Figure S9: Locating docking sites in DNA-PAINT and AFM images. To quantify the quality of correlation between SRM and AFM images, the positions of docking sites were identified independently in SRM images (a) and AFM images (b), depicted by red cross-marks in each image. For DNA-PAINT images, POI were identified within the images and mapped to the localization list to identify all events corresponding to each POI. The position of individual POI were then determined from the average position of the corresponding events. For AFM images, the positions of docking sites were approximated based on the design of the origami. The relative positions and deviations in position for the images are plotted in Figure S10. 

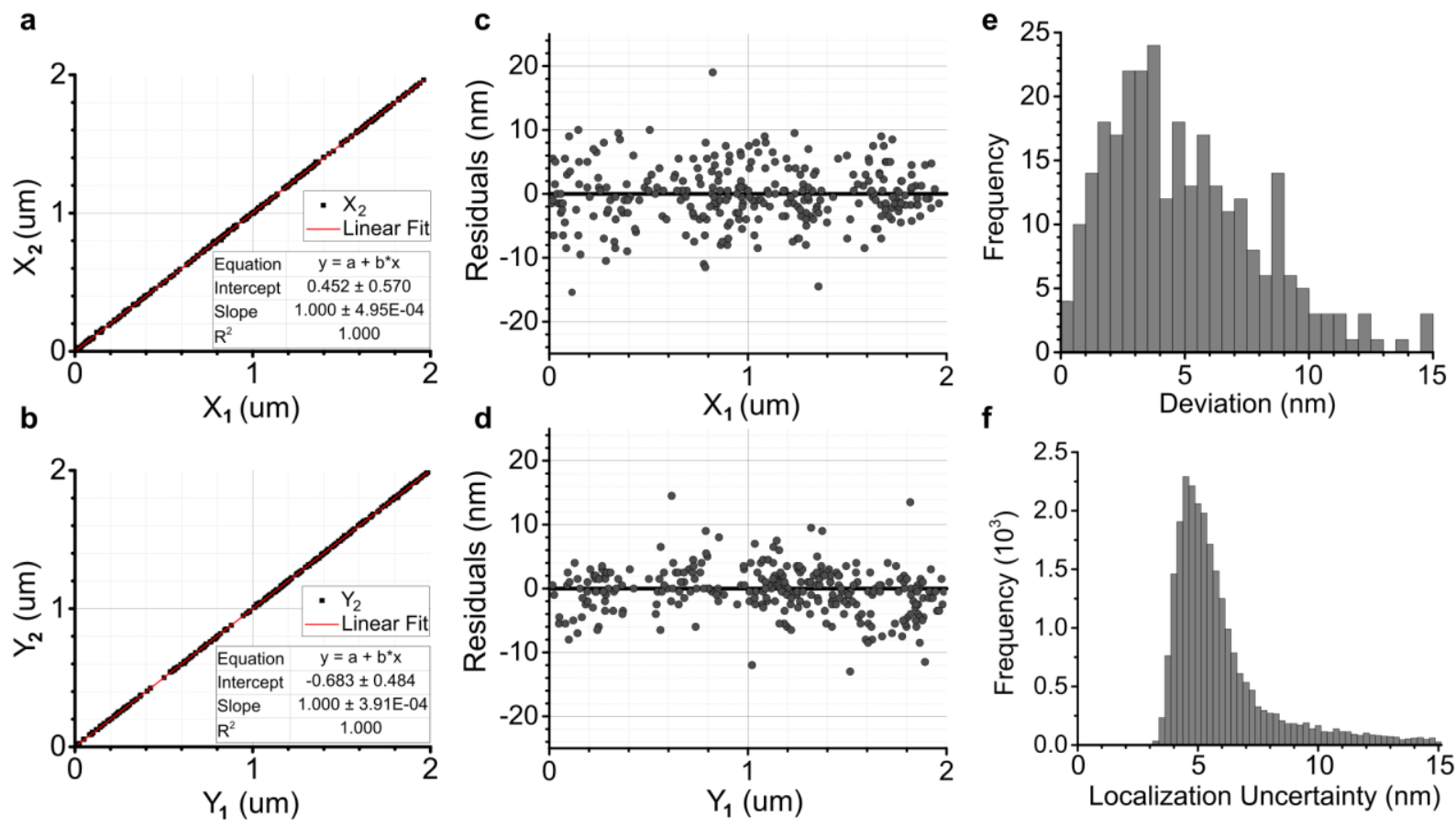

Figure S10: Correlation of docking site positions in AFM and DNA-PAINT images. (a,b) Plots of the $x$ and $y$ coordinates of 286 sites in the AFM images $\left(X_{1}, Y_{1}\right)$ relative to the SRM images $\left(X_{2}, Y_{2}\right)$. The correlation between individual sites within the images is represented by the slope of the fitted lines; for both fits, the slope $b=1.000$ and $R^{2}=1.000$. The results show strong correlation between the images with deviations randomly distributed in the $x$ and $y$ directions. (c,d) Plots of the regular residuals for the fits in $(a, b)$. The residuals provide a better visualization of the position-dependence of deviations between the images. (e) Histogram of the magnitude of deviations for sites within the images. The standard deviation in site positions between the SRM and AFM images (Eq. 3) was $5 \pm 3 \mathrm{~nm}$. (f) Histogram of the localization uncertainty for events in the SRM image corresponding to the correlated images. The root mean square localization uncertainty was $6 \pm 2 \mathrm{~nm}$. 


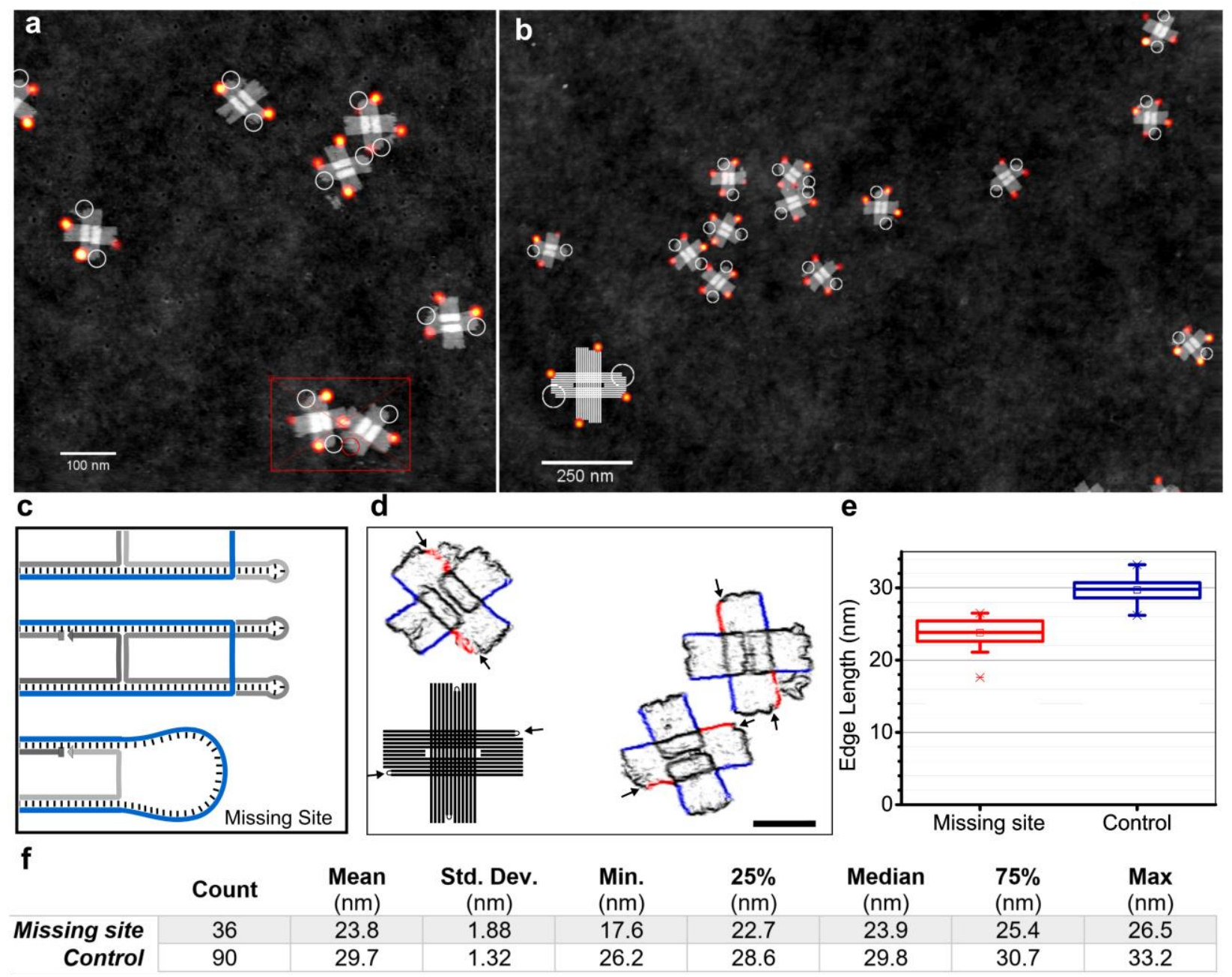

Figure S11: Quantifying unincorporated site detection with AFM. (a,b) Correlative SRM/AFM images of DNA origami cross-tiles synthesized with intentional unincorporated site defects. The DNA-PAINT images were rendered with detections located at the average position of localizations for the associated site to improve visualization. The positions of the defects on individual origami are indicated by the structure diagram in the bottom left of (b), along with simulated SRM detections. Within the image, unincorporated sites which were correctly identified are marked by white circles. One tile arm was obscured by imaging artifacts, but all other unincorporated sites were successfully detected. (c) DNA origami cross-tile strand diagram depicting an unincorporated site defect. (d) To quantify the structural difference between incorporated and unincorporated sites on DNA origami, the edge lengths of DNA origami cross-tiles were measured for intentional unincorporated sites (red) and sites without apparent defects (blue). To aid visualization, the image shown is the inverted local slope of the topography, and unincorporated sites are indicated by black arrows, corresponding to the structure diagram in the bottom left. Scale bar, $50 \mathrm{~nm}$. (e) Box and whisker plots summarizing the distribution of edge lengths for unincorporated sites ('Missing site', red) and incorporated sites ('Control', blue), with a box spanning the 50th percentile and split by the median line, connected to the minimum and maximum values. The mean is indicated by a small box, and one outlier in the missing site measurements is indicated by an ' $x$ '. (f) Table of the values from the box and whisker plot. The means are separated by greater than three standard deviations, indicating a significant difference between the edge length of incorporated and unincorporated sites. For the unincorporated sites examined, the double-stranded helix edge length was expected to be reduced by $5.4 \mathrm{~nm}$ (16 nt) by the defect, in close agreement with the measured $6 \pm 3 \mathrm{~nm}$ difference in the means. 


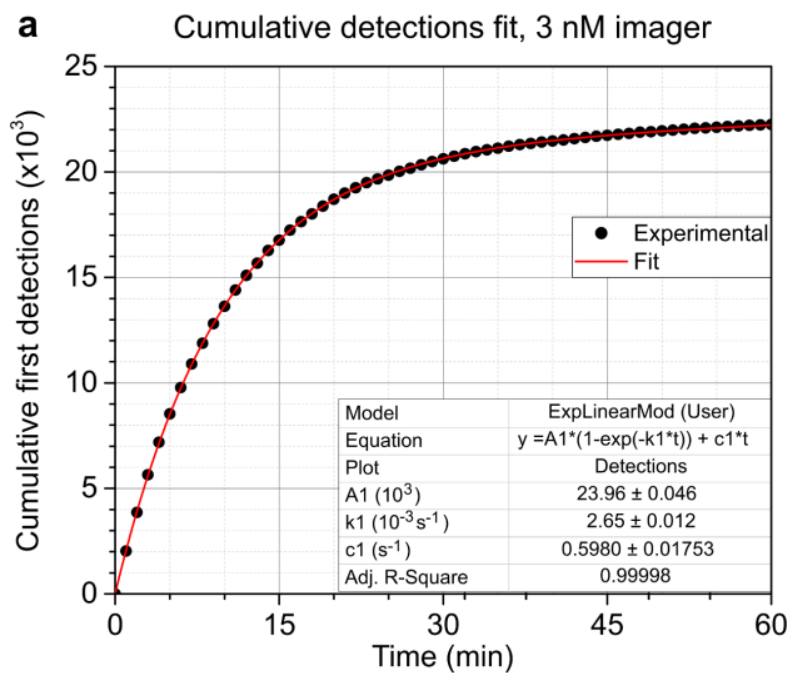

C

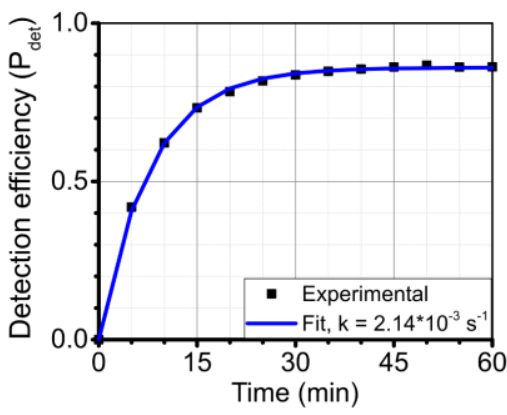

d

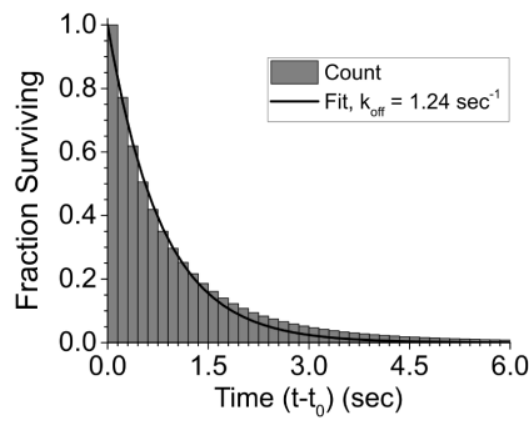

Fit components

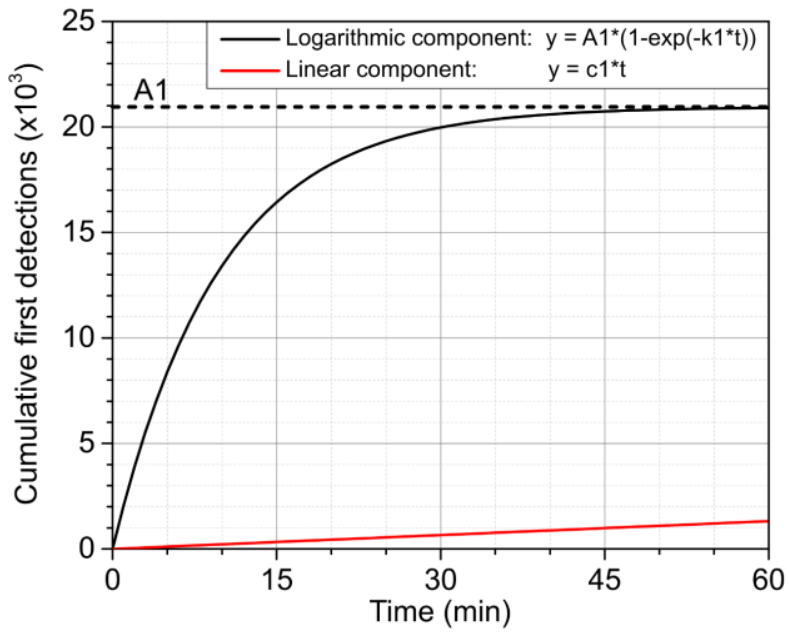

e

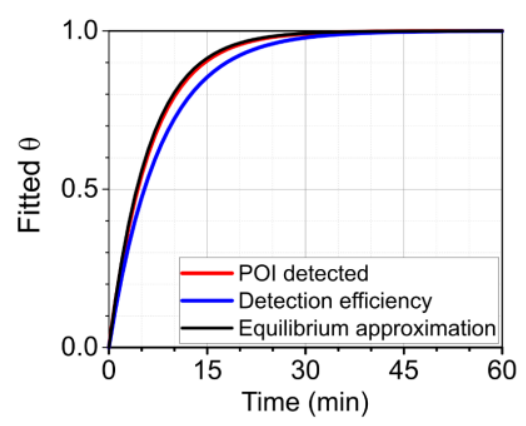

Figure S12: Cumulative first detections of DNA-PAINT sites. (a) Plot of the cumulative first detections for an SRM image of DNA origami cross-tiles acquired in standard imaging buffer with imager strand M1'. The experimental data was fitted to extract the exponential and linear components; the latter was speculated to correspond to non-specific imager strand interactions with the surface. The exponential component was found to have a rate constant of $k_{1}=0.00267 \mathrm{sec}^{-1}$. (b) Plot of the extracted exponential and linear components. The exponential component, representing the cumulative first detections of active docking sites, surpasses $99 \%$ of the predicted population of active sites by 60 minutes of imaging. (c) Plot of the detection efficiency ( $\left.p_{\text {det }}\right)$ measured for SRM images rendered every 5 minutes. For the fitted exponential decay, the rate constant $k=0.00214 \mathrm{sec}^{-1}$. (d) Histogram of fluorescent event lifetimes. The dissociation rate constant $\left(k_{\text {off }}\right)$ was calculated from the exponential decay fit to be $k_{\text {off }}=1.24 \mathrm{sec}^{-1}$. From the list of $\mathrm{POI},\left[I P_{e}\right] /\left[P_{e}\right]$ was found to be 0.0022 , and $k_{1}=0.00277 \mathrm{sec}^{-1}$. (e). Plot comparing the progressions of DNA-PAINT detection predicted with each method. 


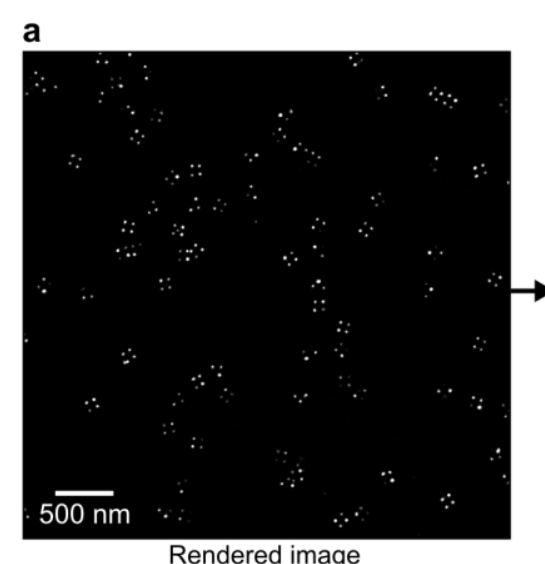

Rendered image

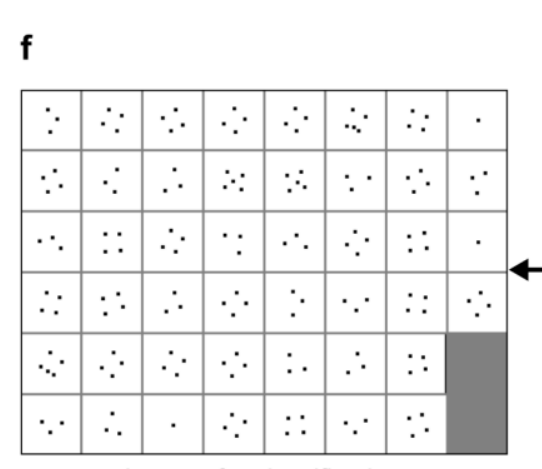

Images for classification b

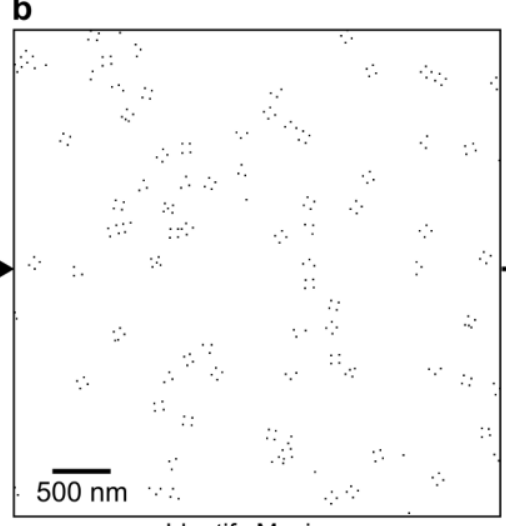

e
Identify Maxima

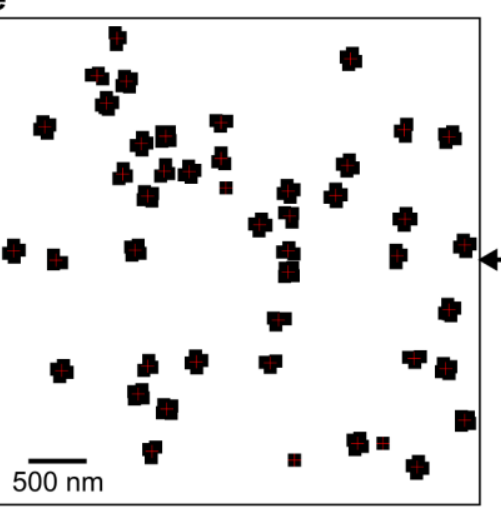

Qualifying features and center of mass

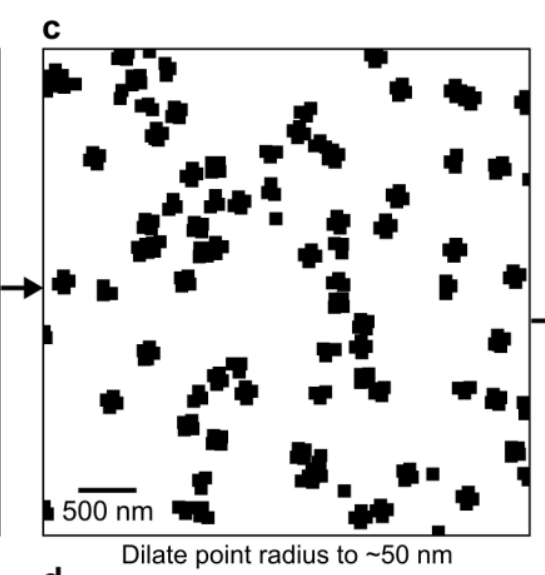

d

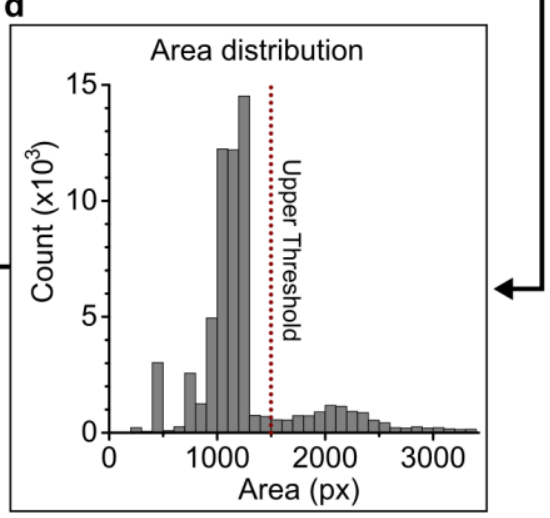

Remove features above threshold

Figure S13: Point-masking procedure for structure classification. (a) DNA-PAINT images were rendered at 20x magnification. (b) Maxima in the images surpassing a threshold of two detections were identified, generating a binary image of maxima. (c) Points within the maxima image were dilated to $50 \mathrm{~nm}$ radius, causing structures within $100 \mathrm{~nm}$ of other structures to be joined. (d) Structures in the dilated image were filtered by area, removing structures with areas larger than expected for a single structure. (e) Filtered image of dilated structures. The center of masses of filtered structures within the image were recorded. (f) The coordinates of filtered structures were used to generate a montage image of structures meeting the criteria for classification. 
a

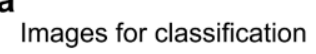

b

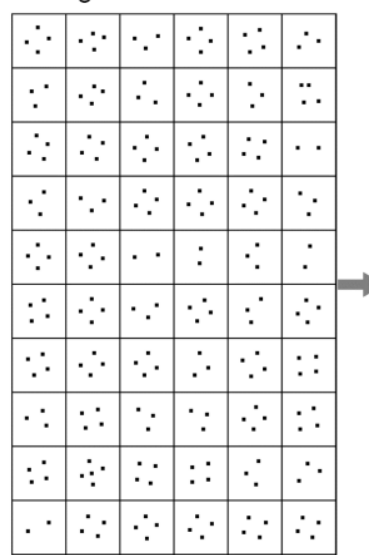
Generation of input vectors

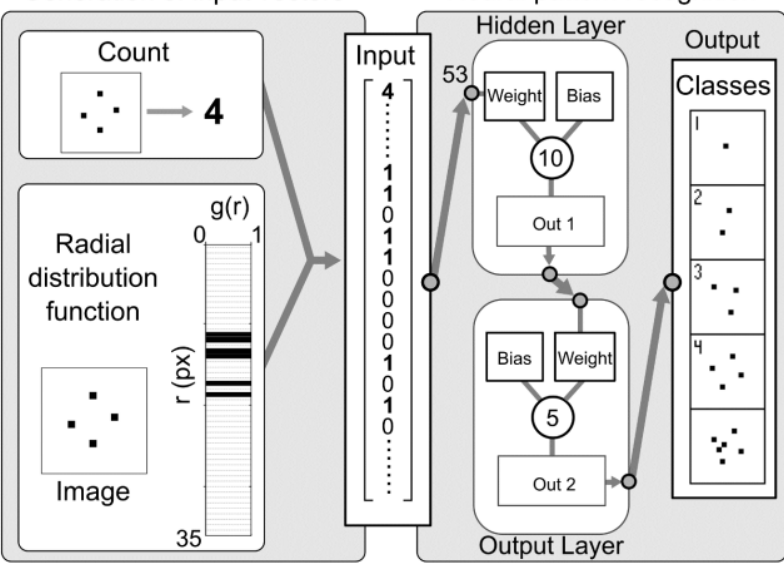

Neural pattern recognition d

Classified images

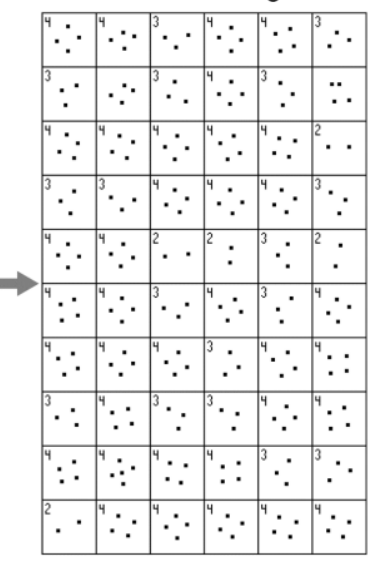

\begin{tabular}{|l|c|c|c|c|c|c|c|c|c|c|}
\cline { 2 - 12 } \multicolumn{1}{c|}{} & Qualifying structures & \multicolumn{2}{c|}{ 2-site structure count } & \multicolumn{2}{c|}{ 3-site structure count } & \multicolumn{2}{c|}{ 4-site structure count } & \multicolumn{2}{c|}{ Detection efficiency } \\
\cline { 2 - 12 } \multicolumn{1}{c|}{} & Automated & Manual & Automated & Manual & Automated & Manual & Automated & Manual & Automated & Manual \\
\hline Sample 1 & 466 & 469 & 33 & 33 & 156 & 157 & 277 & 279 & 0.877 & 0.877 \\
\hline Sample 2 & 418 & 415 & 30 & 30 & 166 & 166 & 222 & 219 & 0.849 & 0.848 \\
\hline Sample 3 & 447 & 449 & 47 & 46 & 175 & 174 & 225 & 229 & 0.839 & 0.842 \\
\hline Sample 4 & 425 & 427 & 68 & 68 & 173 & 174 & 184 & 185 & 0.806 & 0.806 \\
\hline Sample 5 & 353 & 350 & 64 & 60 & 144 & 141 & 145 & 149 & 0.793 & 0.802 \\
\hline
\end{tabular}

Figure S14: Neural pattern recognition for structure classification. (a) Subset of structures identified with the method described in Section B4 and depicted in Figure S13, chosen for automated classification. (b) An input vector is generated for each image. The count of maxima in the image is determined, then the radial distribution is calculated for the image. For the input, the count is vertically concatenated with the radial distribution, generating a row vector for the image. The final input matrix contains the input for individual images separated by column. (c) The inputs are fed into a neural network for classification. The output distinguishes between 5 classes for input images. The network was trained using over 4000 images per class. (d) The montage of input images labelled with the results of classification. The class values represent the number of $\mathrm{POI}$ detected for each structure. With this method, thousands of structures can be classified in seconds. (e) Table of the results of automated and manual counting of five image samples containing 500 structures each. For all five samples, the detection efficiency determined from automated classification was within $1 \%$ of the value obtained by manual classification. 
a

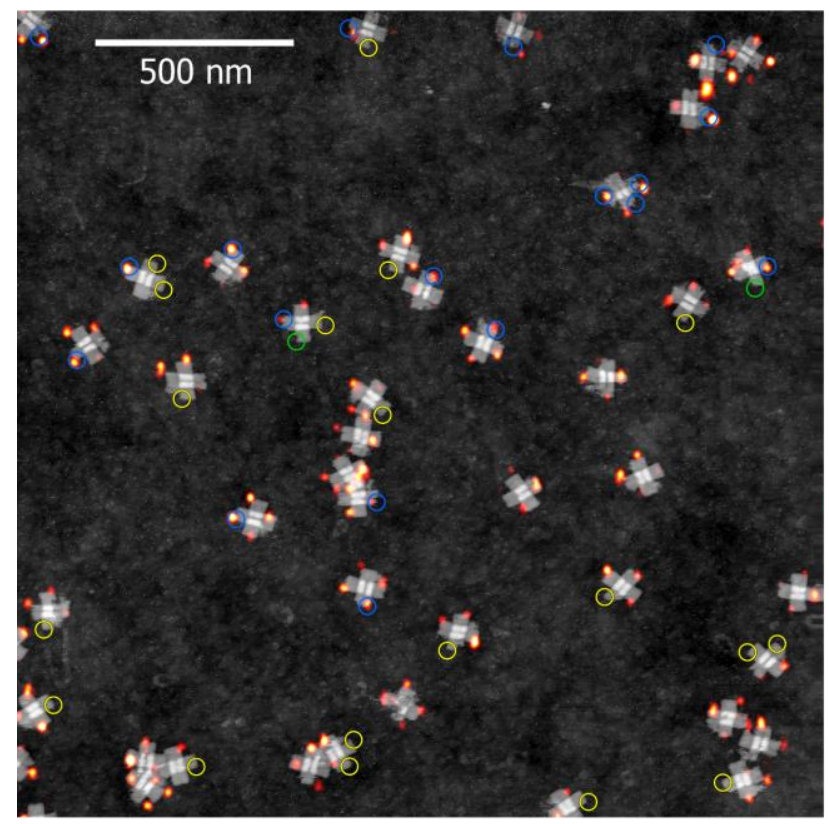

C

\begin{tabular}{|l|c|c|c|c|}
\hline $\mathrm{n}=319$ & SRM & AFM & Count & Distribution \\
\hline Case 1 & $\checkmark$ & $\checkmark$ & 223 & $70 \pm 4 \%$ \\
\hline Case 2 O & $\checkmark$ & $\mathbf{x}$ & 35 & $11 \pm 2 \%$ \\
\hline Case 3 0 & $\mathbf{x}$ & $\checkmark$ & 50 & $16 \pm 3 \%$ \\
\hline Case 4 O & $\mathbf{x}$ & $\mathbf{x}$ & 11 & $3 \pm 3 \%$ \\
\hline
\end{tabular}

b

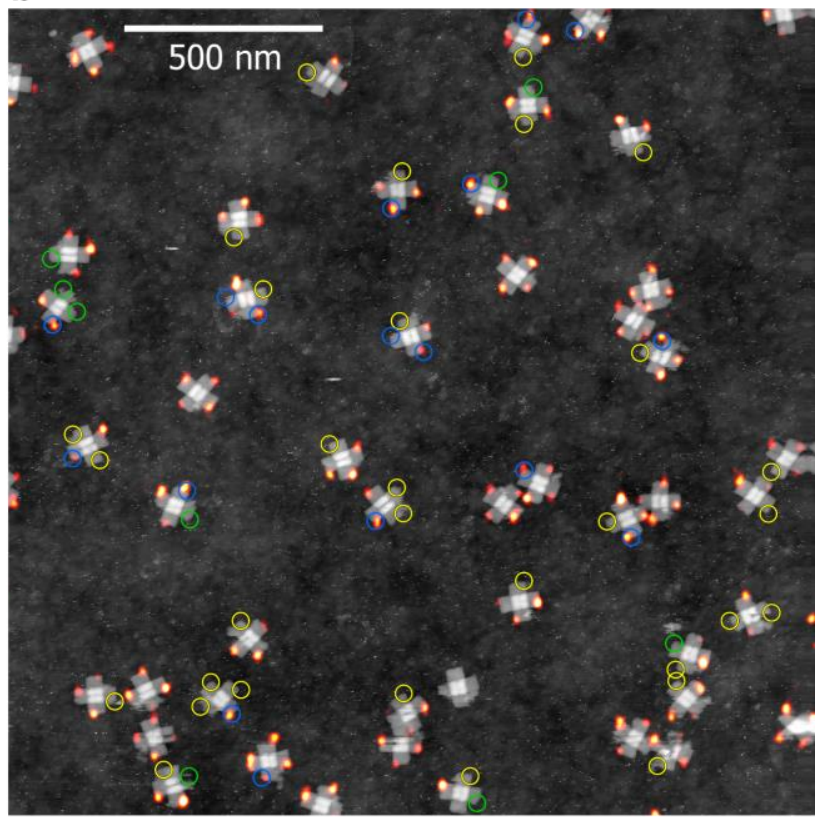

d

\begin{tabular}{|c|c|c|c|c|}
\hline \multirow{2}{*}{ Available sites } & \multirow[t]{2}{*}{ Counts } & \multirow{2}{*}{$\begin{array}{l}\text { Counts } \\
\text { predicted } \\
\text { from fit }\end{array}$} & \multicolumn{2}{|c|}{$\begin{array}{l}\text { Binomial fit } \\
\quad(x=4)\end{array}$} \\
\hline & & & $\mathrm{p}$ & SE \\
\hline 0 & -- & 4 & \multirow{5}{*}{0.822} & \multirow{5}{*}{0.007} \\
\hline 1 & -- & 77 & & \\
\hline 2 & 591 & 532 & & \\
\hline 3 & 1599 & 1637 & & \\
\hline 4 & 1903 & 1887 & & \\
\hline
\end{tabular}

Figure S15: Correlated images and defect metrology of "control" cross-tiles. (a,b) Correlated DNAPAINT/AFM images of cross-tiles containing docking sites on which no additional filtration has been performed. Colored circles indicate AFM defects (Case 2, blue), inactive DNA-PAINT sites (Case 3, yellow), and correlated defects (Case 4, green) manually identified in AFM and SRM images prior to correlation. (c) Table of the results of defect quantification for structures in $(a, b)$. In total, 319 docking sites were examined. Approximately $81 \%$ of docking sites were found to be active (Cases 1 and 2), and $97 \%$ were found to be incorporated (Cases 1,2, and 3). (d) Table of the results of automated structure classification of the full DNA-PAINT image. In total, 4093 structures were classified. The distribution was fitted by a binomial distribution for 4 events with a probability of detection ( $p$ ) of $0.822 \pm 0.007$ per event $-82.2 \pm 0.7 \%$ of docking sites were predicted to be active. 


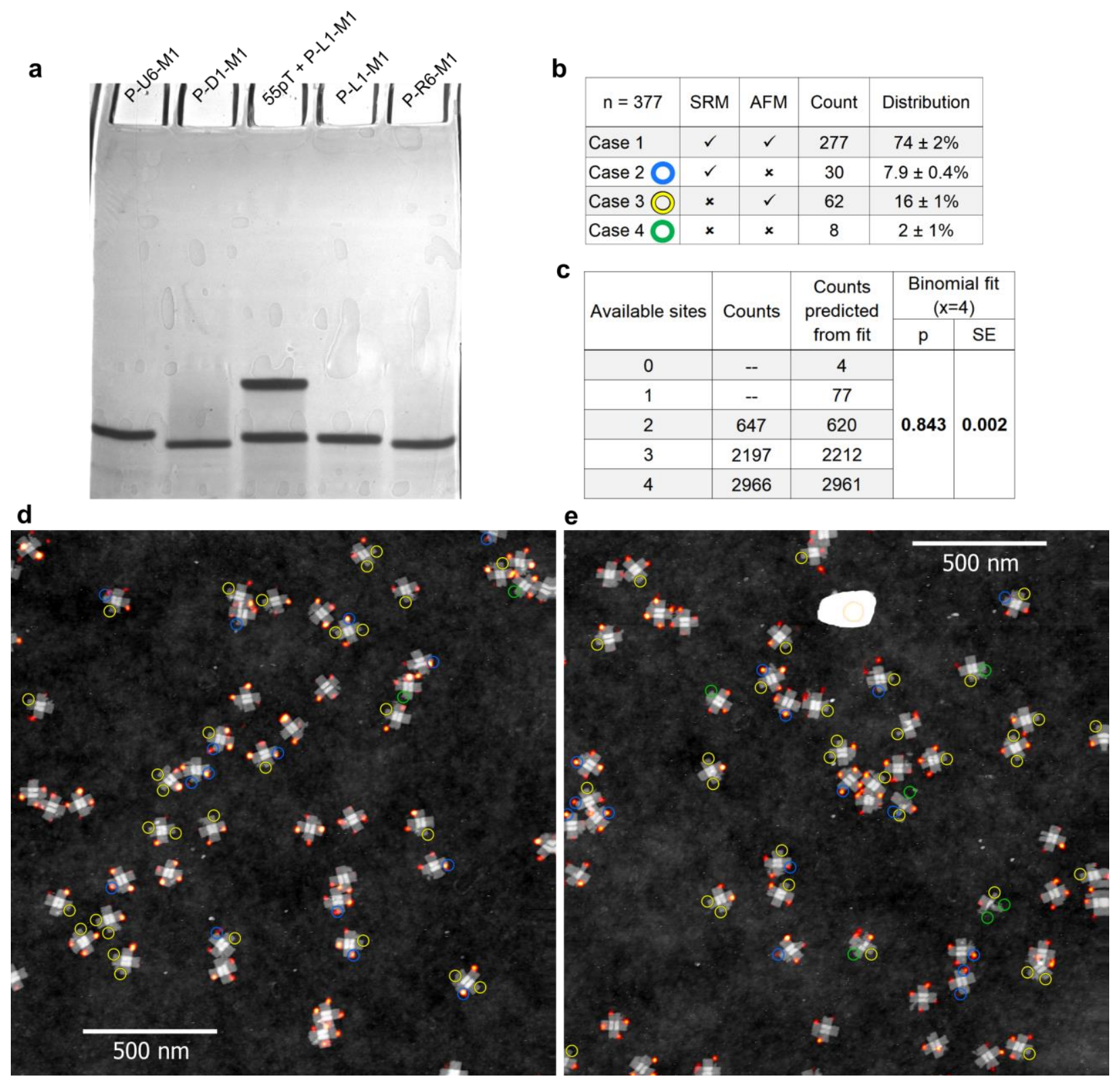

Figure S16: Correlated images and defect metrology of "PAGE-filtered" cross-tiles. (a) PAGE gel image of docking site staple strands in $1 \mathrm{X}$ TBE prior to extraction from the gel; details on procedure and imaging can be found in Section A14. (b) Table of the results of defect quantification for structures in (d,e). In total, 377 docking sites were examined. Approximately $82 \%$ of docking sites were found to be active (Cases 1 and 2), and 98\% were found to be incorporated (Cases 1, 2, and 3). (c) Table of the results of automated structure classification of the full DNA-PAINT image. In total, 5810 structures were classified. The distribution was fitted by a binomial distribution for 4 events with a probability of detection of $0.843 \pm$ 0.002 per event $-84.3 \pm 0.2 \%$ of docking sites were predicted to be active. (d,e) Correlated DNAPAINT/AFM images of cross-tiles containing PAGE-filtered docking sites. Colored circles indicate AFM defects (Case 2, blue), inactive DNA-PAINT sites (Case 3, yellow), and correlated defects (Case 4, green) manually identified in AFM and SRM images prior to correlation. 


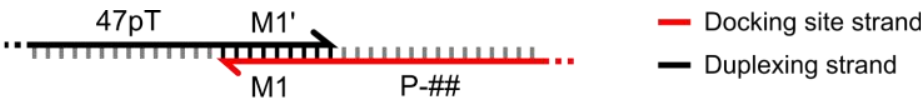

b

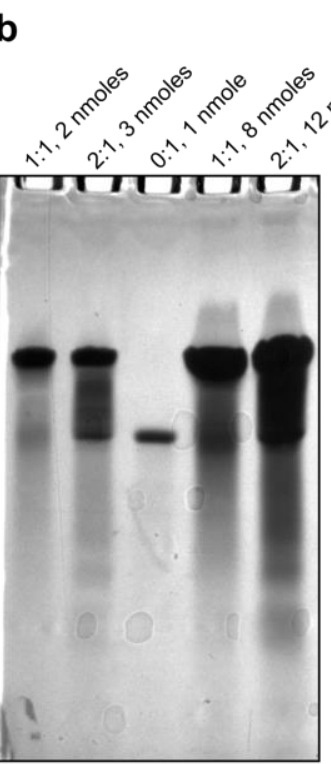

c

f

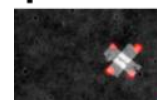

8. 38
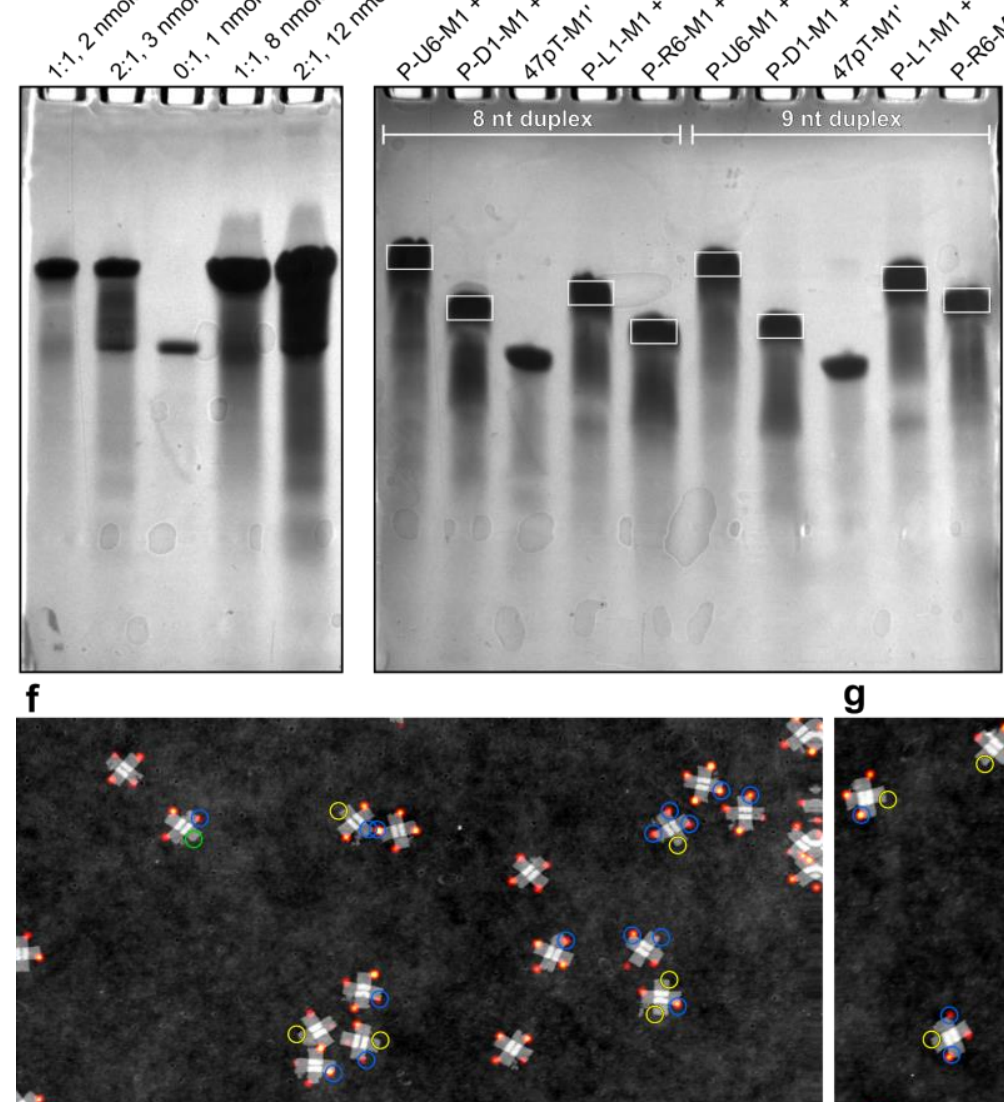
d

\begin{tabular}{|l|c|c|c|c|}
\hline $\mathrm{n}=344$ & SRM & AFM & Count & Distribution \\
\hline Case 1 & $\checkmark$ & $\checkmark$ & 242 & $70 \pm 4 \%$ \\
\hline Case 2 & $\checkmark$ & $\mathbf{x}$ & 55 & $16 \pm 2 \%$ \\
\hline Case 3 & $\mathbf{x}$ & $\checkmark$ & 41 & $12 \pm 3 \%$ \\
\hline Case 4 & $\mathbf{x}$ & $\mathbf{x}$ & 6 & $1.7 \pm 0.5 \%$ \\
\hline
\end{tabular}

e

\begin{tabular}{|c|c|c|c|c|}
\hline \multirow{2}{*}{ Available sites } & \multirow{2}{*}{ Counts } & \multirow{2}{*}{$\begin{array}{c}\text { Counts } \\
\text { predicted } \\
\text { from fit }\end{array}$} & \multicolumn{2}{|c|}{$\begin{array}{c}\text { Binomial fit } \\
(x=4)\end{array}$} \\
\cline { 3 - 4 } & & $p$ & SE \\
\hline 0 & -- & 2 & & \\
\hline 1 & -- & 44 & & \\
\hline 2 & 387 & 403 & \multirow{2}{*}{$\mathbf{0 . 8 6 0}$} & $\mathbf{0 . 0 0 1}$ \\
\hline 3 & 1662 & 1654 & & \\
\hline 4 & 2545 & 2547 & & \\
\hline & & & & \\
\hline
\end{tabular}
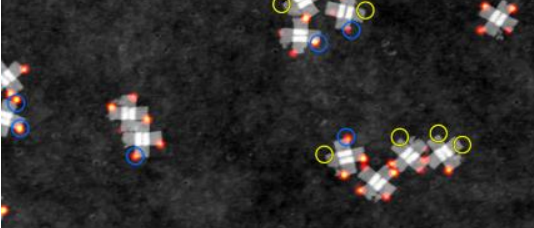

8.
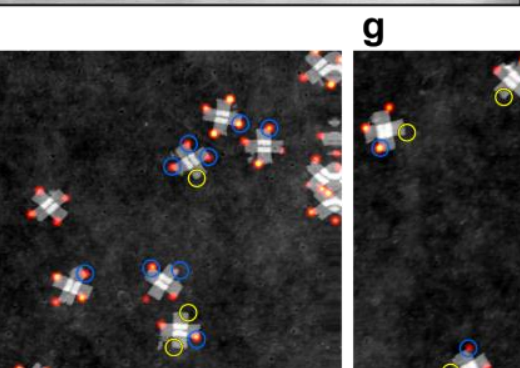

2545

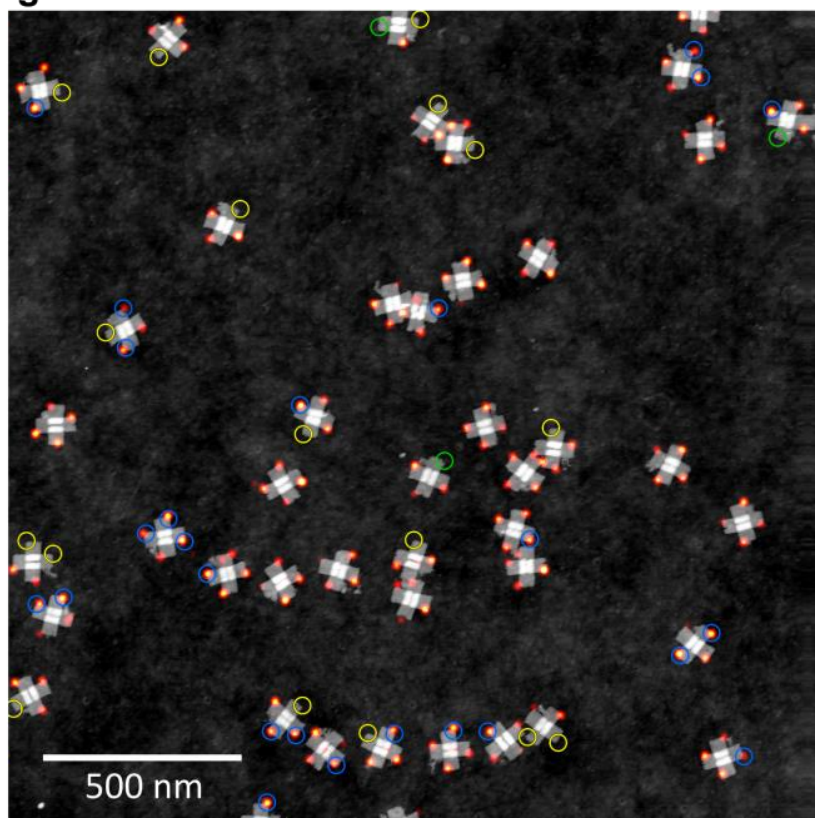

Figure S17: Correlated images and defect metrology of "dpxPAGE-filtered" cross-tiles. (a) Strand diagram depicting docking site strands hybridized to duplexing strands. The $8 \mathrm{nt}$ hybridized domain was found to be stable during PAGE filtration when cooled to $5^{\circ} \mathrm{C}$ in $0.5 \times \mathrm{TBE}$ with $12.5 \mathrm{mM} \mathrm{MgCl}_{2}$. (b) duplexedPAGE filtration of 1:1 and 2:1 strand concentrations [47pT-M1']:[47pt-M1] for 1 nmole and 4 nmole of strand 47pT-M1 added to the wells. The total mass of ssDNA added to each well, from left to right, was $33.4 \mathrm{ng}$, $50.2 \mathrm{ng}, 16.7 \mathrm{ng}, 134 \mathrm{ng}$, and $201 \mathrm{ng}$. Sharp duplexed bands were observed for ratios of 1:1 and 2:1 for up to $50 \mathrm{ng}$ of ssDNA added to the wells. (c) dpxPAGE filtration of docking sites with $8 \mathrm{nt}$ and $9 \mathrm{nt}$ duplexed domains. The duplexing strand 47pT-M1' was included in the $3^{\text {rd }}$ and $8^{\text {th }}$ wells for reference. Docking sites and duplex strands were mixed at $1: 1$ ratio ( 3 nmoles total) and annealed from $90{ }^{\circ} \mathrm{C}$ to $20^{\circ} \mathrm{C}$ over 20 minutes, then the annealed solutions were added to each well. White boxes indicate the bands removed 
from the gel after PAGE filtration. Additional details can be found in Section A15. (d) Table of the results of defect quantification for structures in $(f, g)$. In total, 344 docking sites were examined. Approximately $86 \%$ of docking sites were found to be active (Cases 1 and 2), and $98 \%$ were found to be incorporated (Cases 1,2 , and 3). (e) Table of the results of automated structure classification of the full DNA-PAINT image. In total, 4594 structures were classified. The distribution was fitted by a binomial distribution for 4 events with a probability of detection of $0.860 \pm 0.001$ per event $-86.0 \pm 0.1 \%$ of docking sites were predicted to be active. $(f, g)$ Correlated DNA-PAINT/AFM images with colored circles indicating AFM defects (Case 2, blue), inactive DNA-PAINT sites (Case 3, yellow), and correlated defects (Case 4, green) manually identified in AFM and SRM images prior to correlation.

a

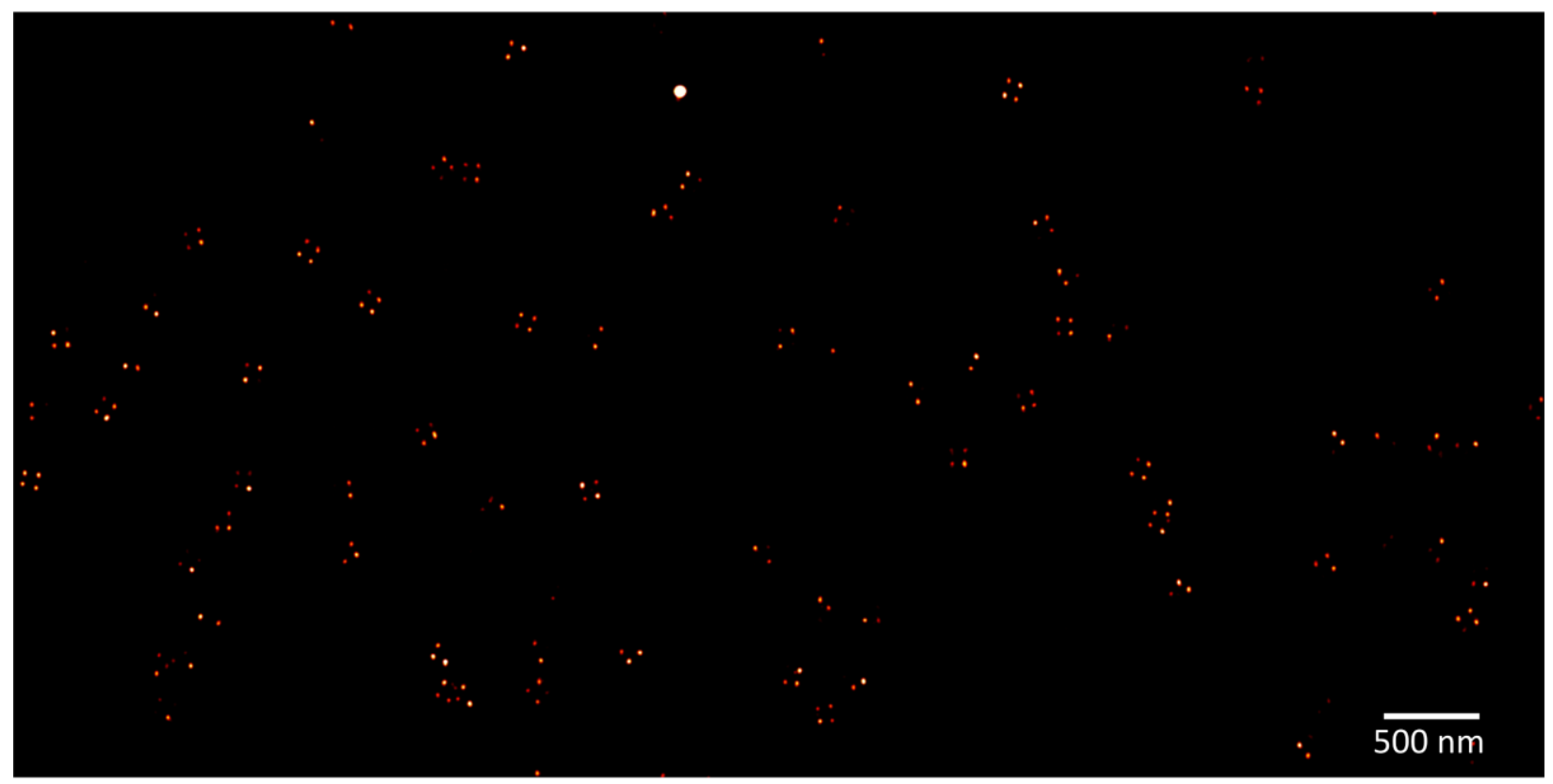

b

\begin{tabular}{|c|c|c|c|c|}
\hline \multirow{2}{*}{ Available sites } & \multirow{2}{*}{ Counts } & $\begin{array}{c}\text { Counts } \\
\text { predicted }\end{array}$ & \multicolumn{2}{|c|}{$\begin{array}{c}\text { Binomial fit } \\
(x=4)\end{array}$} \\
\cline { 3 - 4 } & & from fit & p & SE \\
\hline 0 & -- & 19 & & \\
\hline 1 & -- & 258 & & \\
\hline 2 & 1383 & 1320 & $\mathbf{0 . 7 7 3}$ & $\mathbf{0 . 0 0 6}$ \\
\hline 3 & 2942 & 2997 & & \\
\hline 4 & 2584 & 2552 & & \\
\hline
\end{tabular}

Figure S18: DNA-PAINT analysis of cross-tiles with "n-1 defect" docking sites. (a) DNA-PAINT image of cross-tiles containing $7 \mathrm{nt}$ docking sites, $1 \mathrm{nt}$ shorter than the full $8 \mathrm{nt}$ M1 docking site domain to simulate an $\mathrm{n}-1$ defect. No addition filtration was performed on the docking site strands. (b) Table of the results of automated structure classification of the full DNA-PAINT image. In total, 6909 structures were classified. The distribution was fitted by a binomial distribution for 4 events with a probability of detection of $0.773 \pm$ 0.006 per event $-77.3 \pm 0.6 \%$ of docking sites were predicted to be active. 


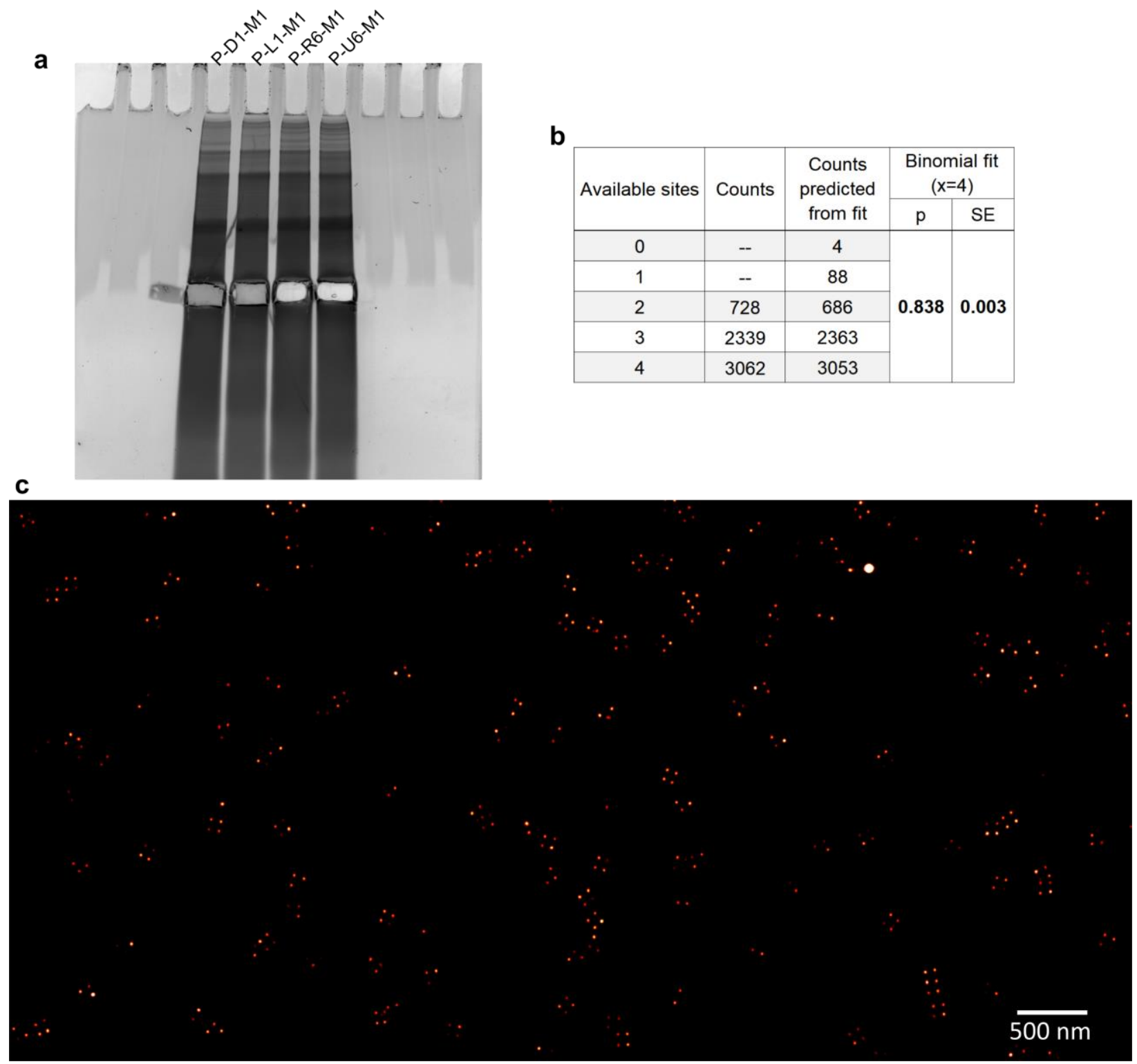

Figure S19: DNA-PAINT analysis of cross-tiles with "Page-filtered, $\mathbf{n}-1$ defect" docking sites. (a) PAGE gel of docking site staple strands with 7 nt docking site domains in $1 \mathrm{X}$ TBE; details on procedure can be found in Section A14. We note that the image was mistakenly acquired after extraction of sample bands from the gel and staining with SYBR® gold. (b) Table of the results of automated structure classification of the full DNA-PAINT image. In total, 6129 structures were classified. The distribution was fitted by a binomial distribution for 4 events with a probability of detection of $0.838 \pm 0.003$ per event -83.8 $\pm 0.3 \%$ of docking sites were predicted to be active. (c) DNA-PAINT image of cross-tiles containing the docking site strands extracted from the gel in (a). 

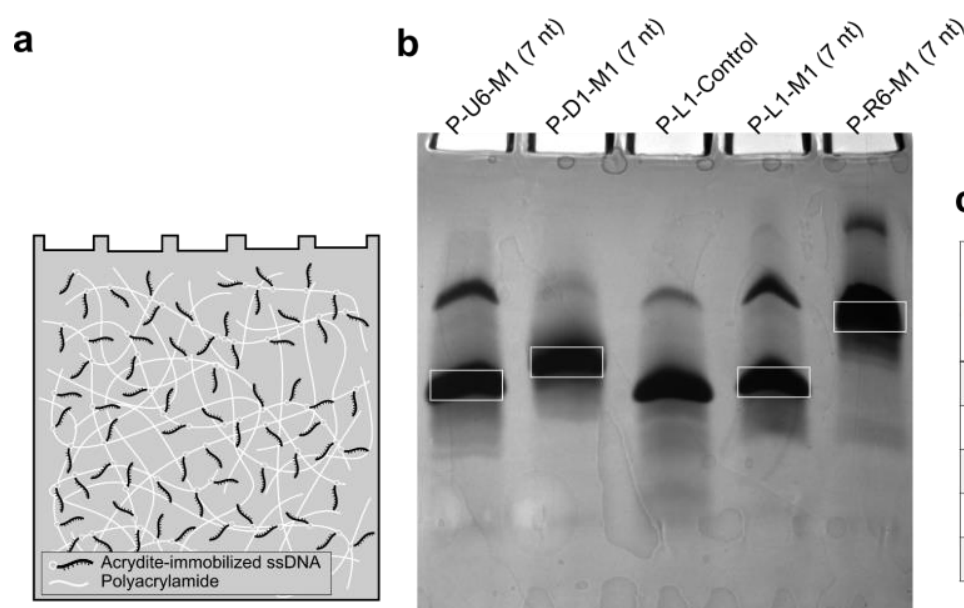

C

d

\begin{tabular}{|c|c|c|c|c|}
\hline \multirow[t]{2}{*}{ Available sites } & \multirow[t]{2}{*}{ Counts } & \multirow{2}{*}{$\begin{array}{l}\text { Counts } \\
\text { predicted } \\
\text { from fit }\end{array}$} & \multicolumn{2}{|c|}{$\begin{array}{l}\text { Binomial fit } \\
\qquad(x=4)\end{array}$} \\
\hline & & & $\mathrm{p}$ & SE \\
\hline 0 & - & 2 & \multirow{5}{*}{0.872} & \multirow{5}{*}{0.002} \\
\hline 1 & - & 50 & & \\
\hline 2 & 549 & 512 & & \\
\hline 3 & 2305 & 2321 & & \\
\hline 4 & 3954 & 3949 & & \\
\hline
\end{tabular}

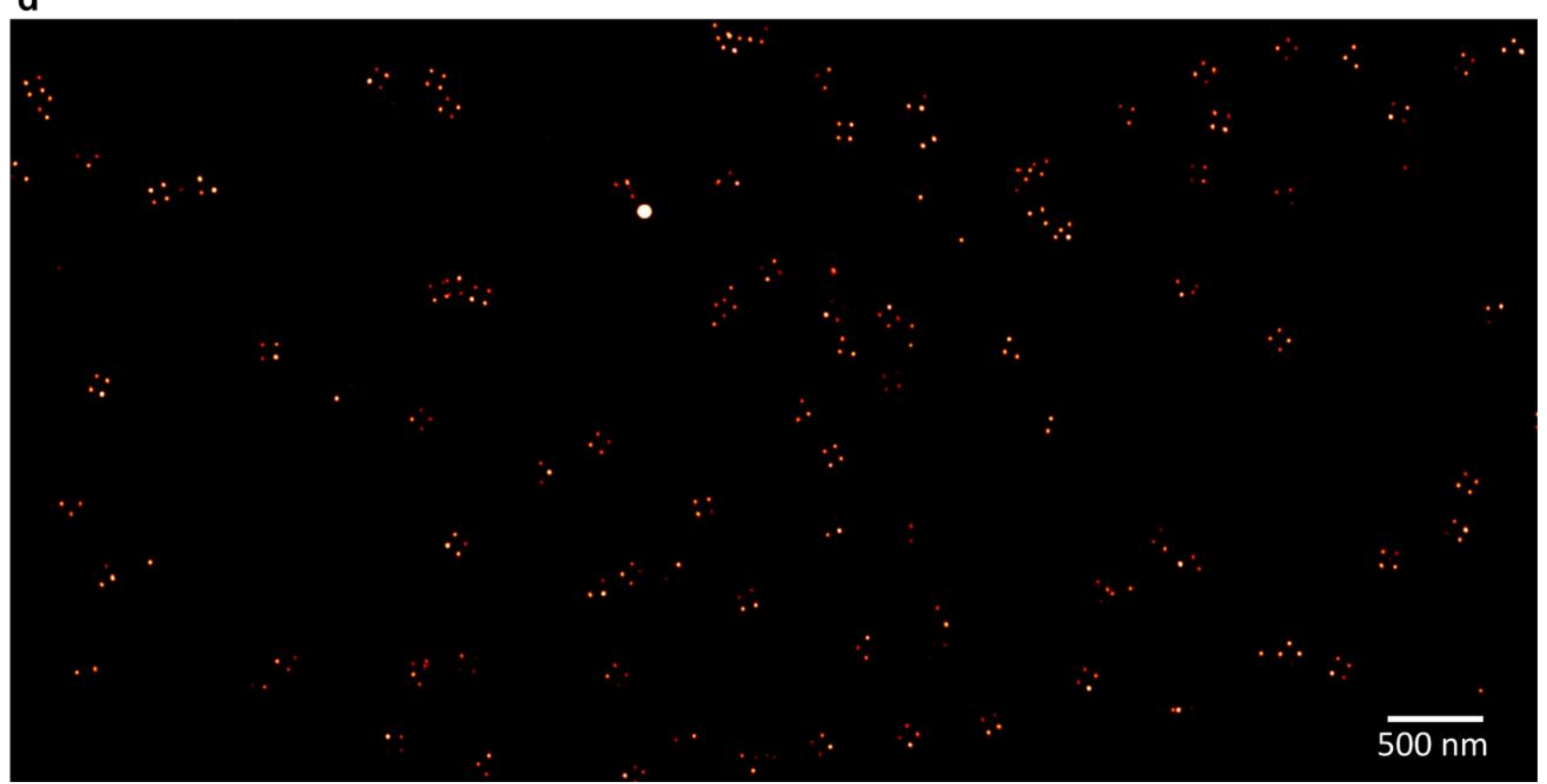

Figure S20: DNA-PAINT analysis of cross-tiles with "seqPAGE-filtered, n-1 defect" docking sites. (a) Depiction of Acrydite-immobilized ssDNA in a polyacrylamide matrix. For docking site-targeted PAGE filtration (seqPAGE), Acrydite-modified sSDNA was incorporated in a polyacrylamide gel at a concentration of $3 \mu \mathrm{m}$. The modified ssDNA polymerizes with acrylamide during polymerization of the gel, immobilizing the strands in the gel. A $7 \mathrm{nt}$ domain of the ssDNA was complementary to docking site M1; migrating strands were speculated to transiently hybridize to immobilized strands if the docking site is present, reducing mobility of the strands through the gel. (b) seqPAGE gel image of docking site strands. 5 nmoles of the indicated strands were added to the wells. White boxes indicate the bands removed from the gel after filtration. Additional details on procedure can be found in Section A16. (c) Table of the results of automated structure classification of the full DNA-PAINT image. In total, 6808 structures were classified. The distribution was fitted by a binomial distribution for 4 events with a probability of detection of $0.872 \pm 0.002$ per event $-87.2 \pm 0.2 \%$ of docking sites were predicted to be active. (d) DNA-PAINT image of cross-tiles containing the docking site strands extracted from the gel in (b). 

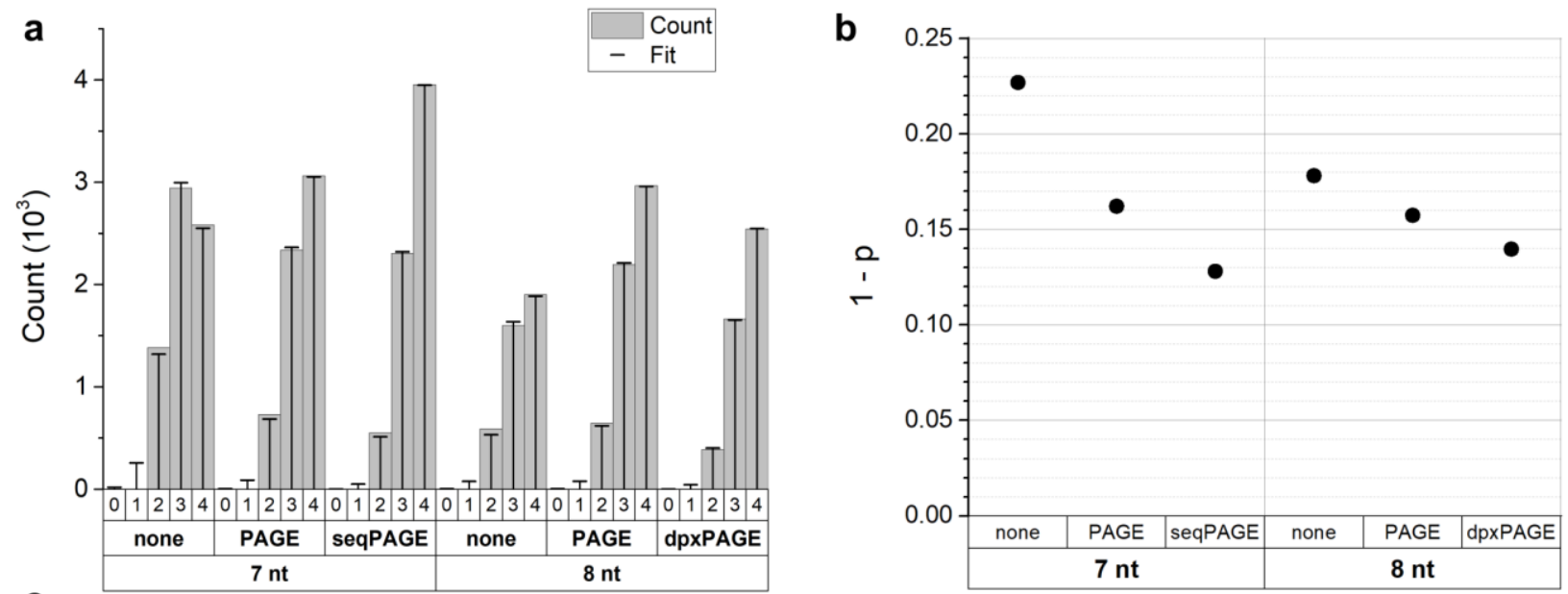

C

\begin{tabular}{|c|c|c|c|c|c|c|c|c|c|c|c|c|c|c|}
\hline \multirow{2}{*}{ Length } & \multirow{2}{*}{ Filtration } & \multirow{2}{*}{ Total count } & \multicolumn{5}{|c|}{ Active sites per structure } & \multicolumn{2}{|c|}{ Binomial fit } & \multicolumn{5}{|c|}{ Fitted active sites per structure } \\
\hline & & & 0 & 1 & 2 & 3 & 4 & $\mathrm{p}$ & Total count & 0 & 1 & 2 & 3 & 4 \\
\hline \multirow{3}{*}{$7 \mathrm{nt}$} & none & 6909 & - & -- & 1383 & 2942 & 2584 & 0.773 & 7146 & 19 & 258 & 1320 & 2997 & 2552 \\
\hline & PAGE & 6129 & -- & -- & 728 & 2339 & 3062 & 0.838 & 6195 & 4 & 88 & 686 & 2363 & 3053 \\
\hline & seqPAGE & 6808 & - & -- & 549 & 2305 & 3954 & 0.872 & 6834 & 2 & 50 & 512 & 2321 & 3949 \\
\hline \multirow{3}{*}{$8 \mathrm{nt}$} & none & 4093 & -- & -- & 591 & 1599 & 1903 & 0.822 & 4137 & 4 & 77 & 532 & 1637 & 1887 \\
\hline & PAGE & 5810 & -- & - & 647 & 2197 & 2966 & 0.843 & 5873 & 4 & 77 & 620 & 2212 & 2961 \\
\hline & dpxPAGE & 4594 & -- & -- & 387 & 1662 & 2545 & 0.860 & 4650 & 2 & 44 & 403 & 1654 & 2547 \\
\hline
\end{tabular}

Figure S21: Summary of results of strand filtration techniques. Six sets of DNA origami cross-tiles were synthesized with $7 \mathrm{nt}$ or $8 \mathrm{nt}$ docking sites with no filtration, PAGE-filtration, seqPAGE-filtration (7 nt only), or dpxPAGE-filtration (8 nt only). DNA-PAINT imaging was performed on each set and classified to calculate the detection efficiency $(p)$ for docking sites filtered by each method. (a) Histogram of the experimental counts of classified structures with two, three, or four active docking sites (grey bars), and the counts expected for a binomial distribution fitted to the results (black bars). The parameters of the binomial distribution for each sample can be found in (c). (b) Plot of the rate of inactive sites (1-p) for each origami set. For both $7 \mathrm{nt}$ and $8 \mathrm{nt}$ docking sites, the rate of defects decreased after PAGE-filtration, and the lowest rates of defects were achieved for the docking site-targeted filtration methods. (c) Table of the counting results and fits for each sample. 
a

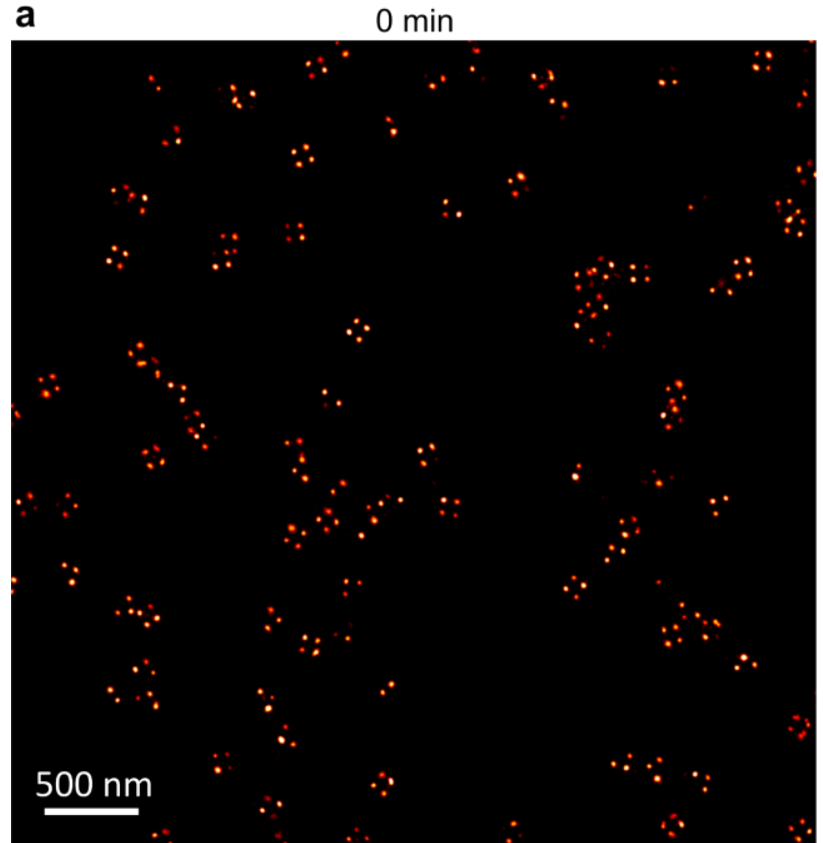

c

$3 \min$

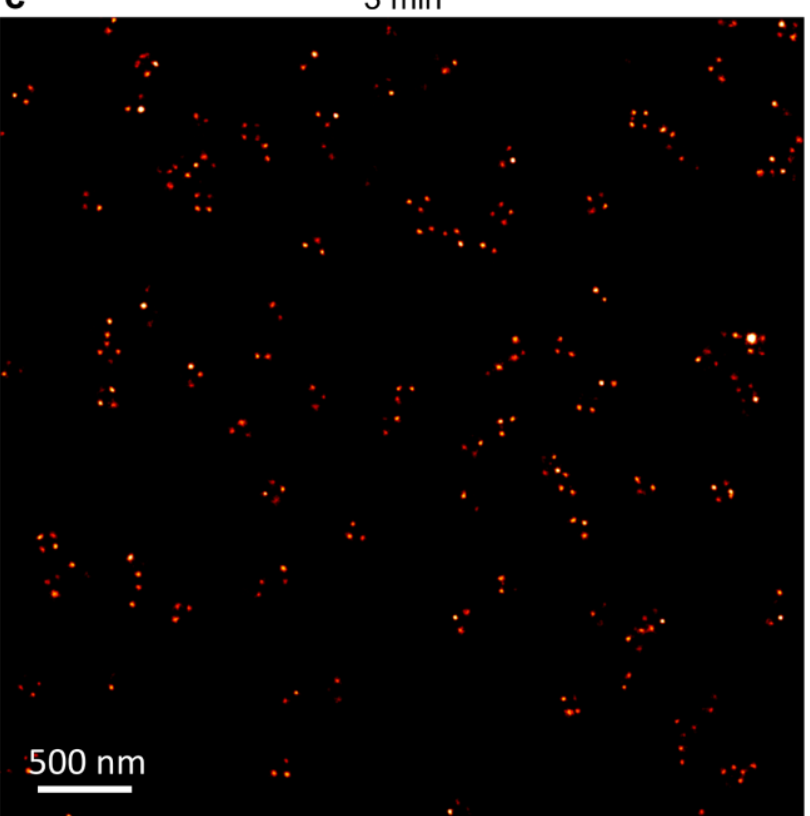

b

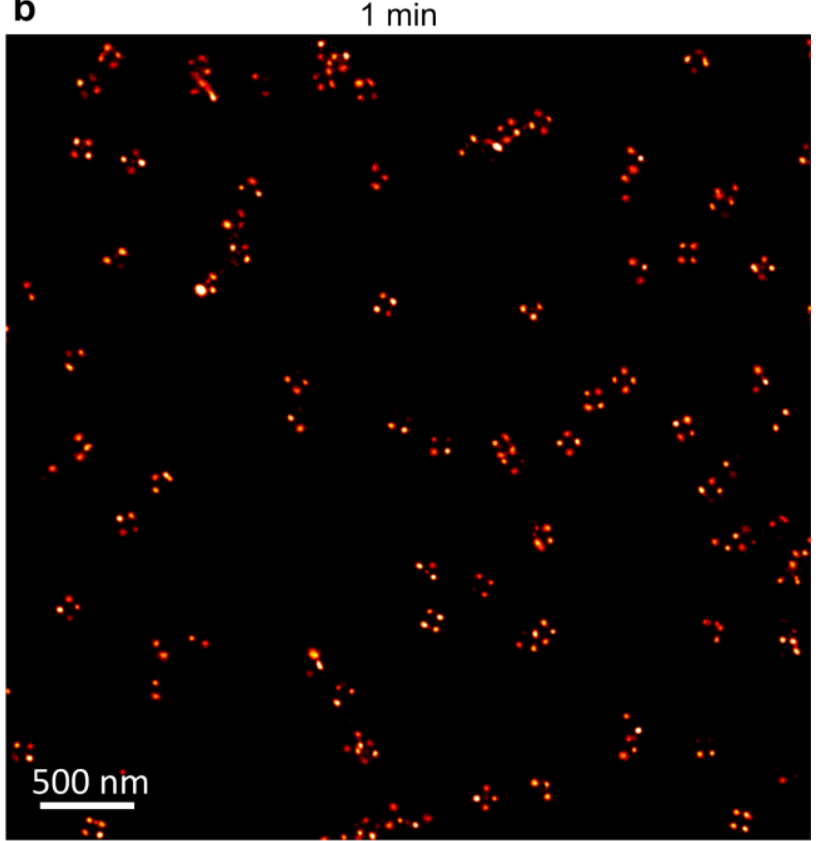

d

$5 \min$

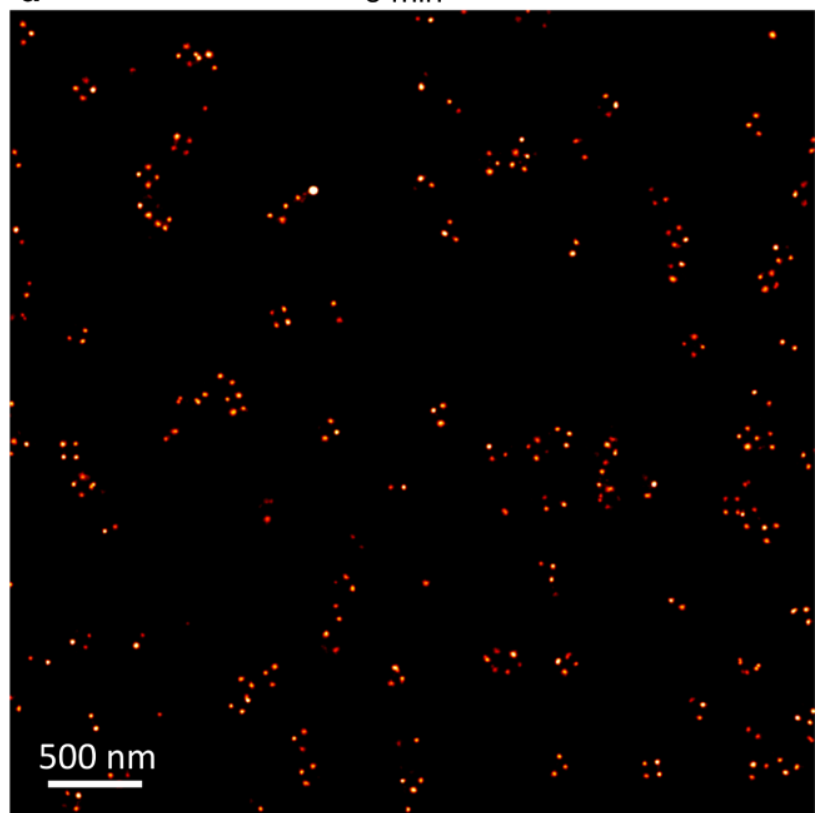

Figure S22: DNA-PAINT images of DNA origami cross-tiles with varying duration of UV exposure. DNA-PAINT images of DNA origami cross-tiles exposed to $305 \mathrm{~nm}$ UV for $0 \mathrm{~min}(\mathrm{a}), 1 \mathrm{~min}$ (b), $3 \mathrm{~min}$ (c), and $5 \mathrm{~min}$ (d). Oxygen scavengers ( $\mathrm{PO}+\mathrm{C}$ and trolox) were included in all samples, and imaging was performed in sealed microchannels. Automated structure classification was performed on each sample; the results are provided in Figure S23. 


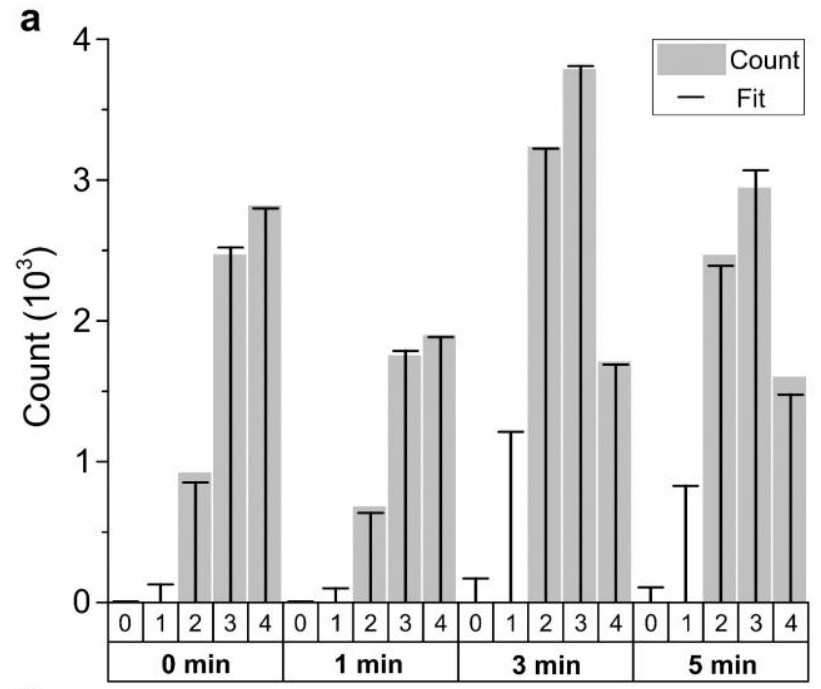

b

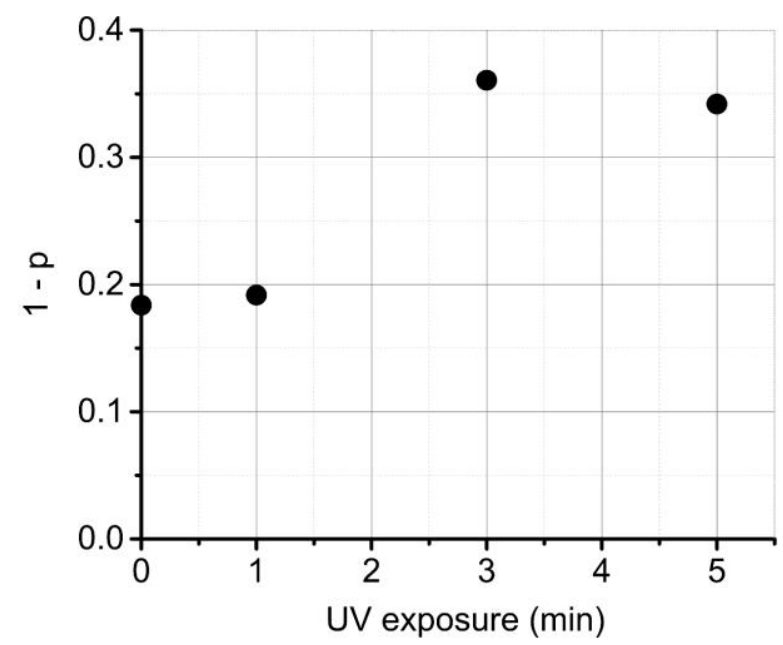

C

\begin{tabular}{|c|c|c|c|c|c|c|c|c|c|c|c|c|c|c|}
\hline \multirow{2}{*}{$\begin{array}{c}\text { UV } \\
\text { exposure }\end{array}$} & \multirow{2}{*}{$\begin{array}{l}\text { Total } \\
\text { count }\end{array}$} & \multicolumn{5}{|c|}{ Active sites per structure } & \multicolumn{3}{|c|}{ Binomial fit } & \multicolumn{5}{|c|}{ Fitted active sites per structure } \\
\hline & & 0 & 1 & 2 & 3 & 4 & $\mathrm{p}$ & SE & Total count & 0 & 1 & 2 & 3 & 4 \\
\hline $0 \min$ & 6216 & -- & -- & 924 & 2472 & 2820 & 0.816 & 0.006 & 6305 & 7 & 128 & 851 & 2520 & 2798 \\
\hline $1 \mathrm{~min}$ & 4337 & -- & -- & 683 & 1754 & 1900 & 0.808 & 0.006 & 4414 & 6 & 101 & 636 & 1787 & 1884 \\
\hline $3 \mathrm{~min}$ & 8735 & -- & -- & 3235 & 3789 & 1711 & 0.639 & 0.002 & 10103 & 171 & 1212 & 3223 & 3809 & 1689 \\
\hline $5 \mathrm{~min}$ & 7021 & -- & -- & 2471 & 2946 & 1604 & 0.66 & 0.02 & 7873 & 108 & 828 & 2392 & 3069 & 1477 \\
\hline
\end{tabular}

Figure S23: Summary of results of UV exposure experiments. DNA origami cross-tiles with unfiltered docking sites were exposed to $305 \mathrm{~nm}$ UV light on a tabletop illuminator for varying lengths of time. DNAPAINT imaging was performed on each set and classified to calculate the detection efficiency $(p)$ for docking sites filtered by each method. (a) Histogram of the experimental counts of classified structures with two, three, or four active docking sites (grey bars) and the counts expected for a binomial distribution fitted to the results (black bars). The parameters of the binomial distribution for each sample can be found in (c). (b) Plot of the rate of inactive sites (1-p) for each origami set. For both $7 \mathrm{nt}$ and $8 \mathrm{nt}$ docking sites, the rate of defects decreased after PAGE-filtration, and the lowest rates of defects were achieved for the docking site-targeted filtration methods. 
a

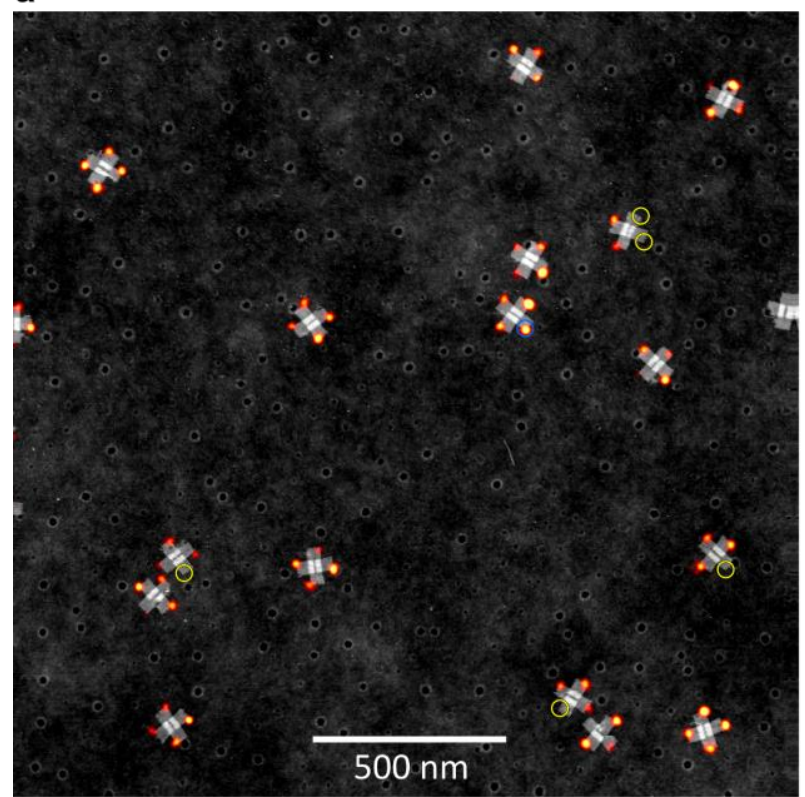

C

\begin{tabular}{|l|c|c|c|c|}
\hline $\mathrm{n}=147$ & SRM & AFM & Count & Distribution \\
\hline Case 1 & $\checkmark$ & $\checkmark$ & 129 & $86 \pm 5 \%$ \\
\hline Case 2 & $\checkmark$ & $\mathbf{x}$ & 7 & $5 \pm 4 \%$ \\
\hline Case 3 & $\mathbf{x}$ & $\checkmark$ & 12 & $8 \pm 1 \%$ \\
\hline Case 4 & $\mathbf{x}$ & $\mathbf{x}$ & 1 & $1 \pm 1 \%$ \\
\hline
\end{tabular}

b

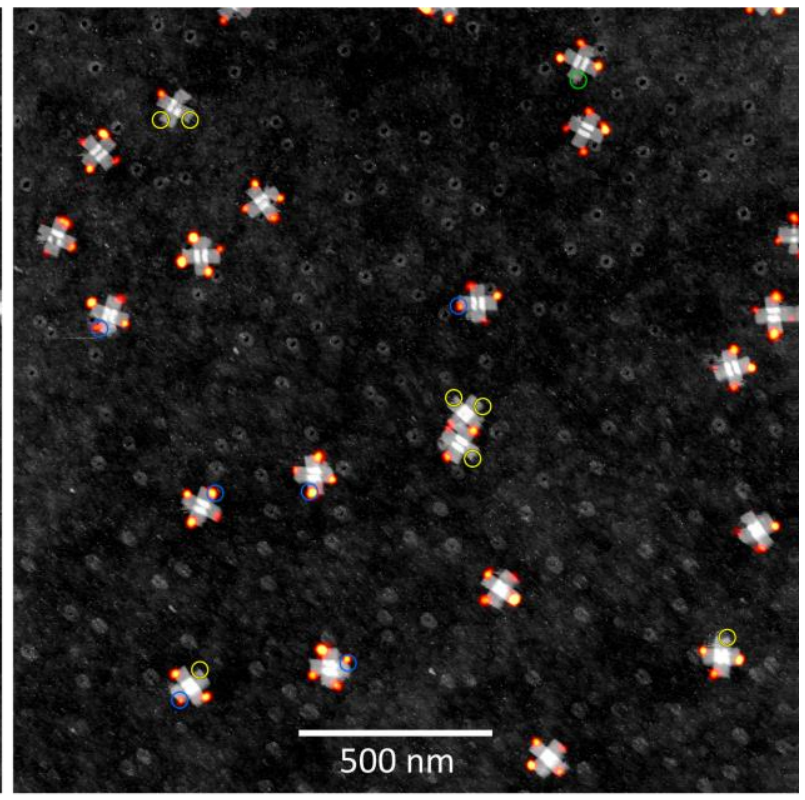

d

\begin{tabular}{|c|c|c|c|c|}
\hline \multirow{2}{*}{ Available sites } & \multirow{2}{*}{ Counts } & \multirow{2}{*}{$\begin{array}{c}\text { Counts } \\
\text { predicted } \\
\end{array}$} & & \multicolumn{2}{|c|}{ Binomial fit $(x=4)$} \\
\cline { 3 - 3 } & & from fit & $p$ & SE \\
\hline 0 & - & 3 & & \\
\cline { 1 - 3 } & -- & 71 & \multirow{2}{*}{0.852} & \multirow{2}{*}{0.008} \\
\hline 2 & 734 & 611 & & \\
\hline 3 & 2277 & 2340 & & \\
\hline 4 & 3383 & 3361 & & \\
\hline
\end{tabular}

Figure S24: Correlated images and defect metrology of "control" cross-tiles. (a,b) Correlated DNAPAINT/AFM images of cross-tiles containing docking sites on which no additional filtration has been performed. The docking site strands used in these structures were newly purchased and are distinct from the "control" cross-tiles in Figure S18. DNA-PAINT imaging was performed in a sealed microchannel for comparison to samples containing oxygen scavengers. Cross-tiles were exposed to $305 \mathrm{~nm}$ UV for $1 \mathrm{~min}$ on a tabletop illuminator to simulate the exposure typically encountered while cutting sample bands from the agarose gel. In the correlated images, colored circles indicate AFM defects (Case 2, blue), inactive DNA-PAINT sites (Case 3, yellow), and correlated defects (Case 4, green) manually identified in AFM and SRM images prior to correlation. (c) Table of the results of defect quantification for structures in $(a, b)$. In total, 149 docking sites were examined. Approximately $91 \%$ of docking sites were found to be active (Cases 1 and 2), and $99 \%$ were found to be incorporated (Cases 1, 2, and 3). AFM image quality degraded quicking during characterization, limiting the number of origami that could be characterized in correlative metrology. (d) Table of the results of automated structure classification of the full DNA-PAINT image. In total, 6394 structures were classified. The distribution was fitted by a binomial distribution for 4 events with a probability of detection ( $p$ ) of $0.852 \pm 0.008$ per event $-85.2 \pm 0.8 \%$ of docking sites were predicted to be active. 
a

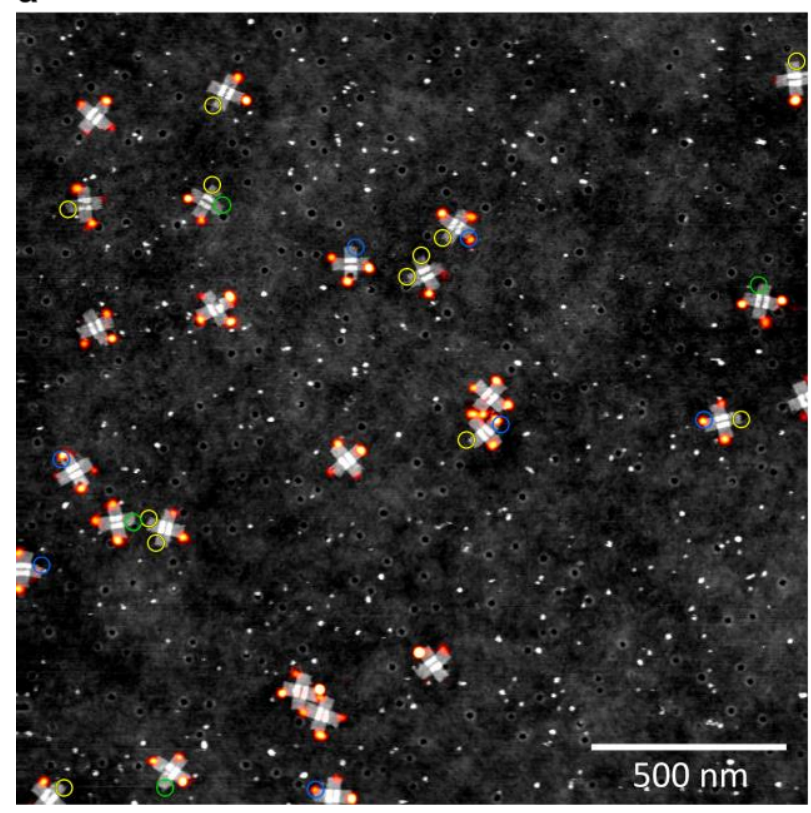

C

\begin{tabular}{|l|c|c|c|c|}
\hline $\mathrm{n}=184$ & SRM & AFM & Count & Distribution \\
\hline Case 1 & $\checkmark$ & $\checkmark$ & 137 & $74 \pm 1 \%$ \\
\hline Case 2 O & $\checkmark$ & $\times$ & 17 & $9 \pm 2 \%$ \\
\hline Case 3 0 & $\times$ & $\checkmark$ & 22 & $12 \pm 2 \%$ \\
\hline Case 4 O & $\times$ & $\times$ & 8 & $4.3 \pm 0.2 \%$ \\
\hline
\end{tabular}

b

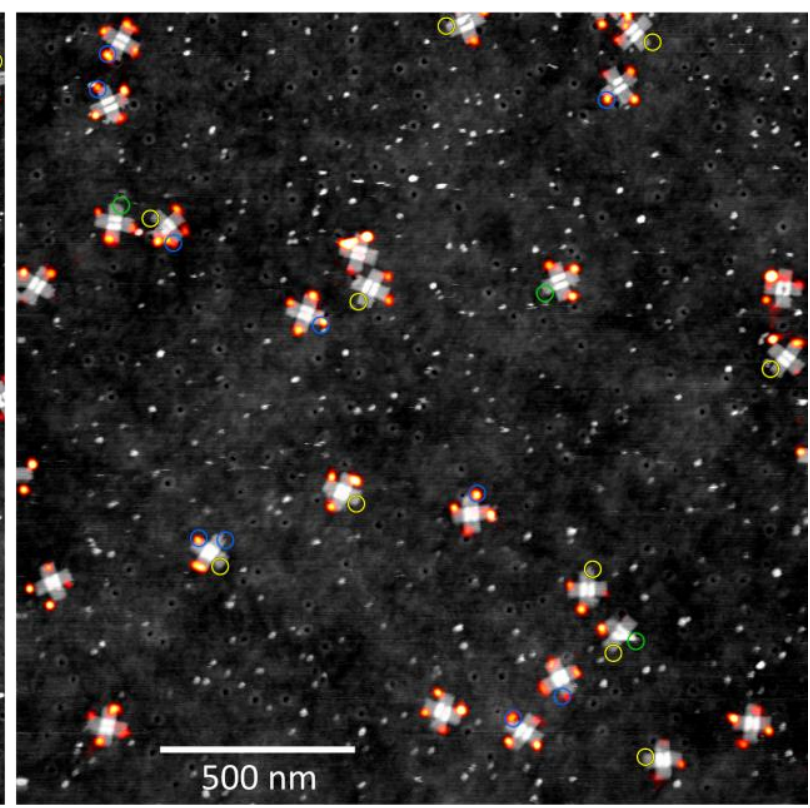

d

\begin{tabular}{|c|c|c|c|c|}
\hline \multirow{2}{*}{ Available sites } & \multirow{2}{*}{ Counts } & \multirow{2}{*}{\begin{tabular}{|c|} 
Counts \\
predicted \\
from fit \\
\end{tabular}} & \multicolumn{2}{|c|}{ Binomial fit $(x=4)$} \\
\hline & & & $p$ & SE \\
\hline 0 & - & 6 & \multirow{5}{*}{0.808} & \multirow{5}{*}{0.006} \\
\hline 1 & - & 101 & & \\
\hline 2 & 683 & 636 & & \\
\hline 3 & 1754 & 1787 & & \\
\hline 4 & 1900 & 1884 & & \\
\hline
\end{tabular}

Figure S25: Correlated images and defect metrology of "control" cross-tiles with oxygen scavengers. $(a, b)$ Correlated DNA-PAINT/AFM images of cross-tiles containing unfiltered docking sites and oxygen scavengers ( $\mathrm{PO}+\mathrm{C}$ and trolox) in solution. The cross-tiles imaged were identical to those in Figure S27. DNA-PAINT imaging was performed in a sealed microchannel for comparison to samples containing oxygen scavengers. Cross-tiles were exposed to $305 \mathrm{~nm}$ UV for $1 \mathrm{~min}$ on a tabletop illuminator to simulate the exposure typically encountered while cutting sample bands from the agarose gel. In the correlated images, colored circles indicate AFM defects (Case 2, blue), inactive DNA-PAINT sites (Case 3, yellow), and correlated defects (Case 4, green) manually identified in AFM and SRM images prior to correlation. The enzymes could be observed on the surface during AFM imaging, appearing as white spots within the images. (c) Table of the results of defect quantification for structures in (a,b). In total, 184 docking sites were examined. Approximately $83 \%$ of docking sites were found to be active (Cases 1 and 2 ), and $96 \%$ were found to be incorporated (Cases 1,2, and 3). (d) Table of the results of automated structure classification of the full DNA-PAINT image. In total, 4337 structures were classified. The distribution was fitted by a binomial distribution for 4 events with a probability of detection (p) of $0.808 \pm 0.006$ per event $80.8 \pm 0.6 \%$ of docking sites were predicted to be active. 


\section{Supporting Tables}

Table S1: List of internal staples for DNA origami cross-tile.

\begin{tabular}{|c|c|c|}
\hline Name & Sequence & Notes \\
\hline LR-A1 & GACAACTCGTATTAAAACTTTACAAACAATTC & \\
\hline LR-A2 & AGTACCTTTTACATCGATGAATATACAGTAAC & \\
\hline LR-A3 & GATAGCTTAGATTAAGTCCTTGAAAACATAGC & \\
\hline LR-A4 & AACGAGAATGACCATATTTAAACAGTTCAGAA & \\
\hline LR-A5 & AAAGTACGGTGTCTGGTTTTAAATATGCAACT & \\
\hline LR-A6 & TGTAATACTTTTGCGGAAAAACATTATGACCC & \\
\hline LR-B1 & GTATTAGTCCTTTGCCCGAACGCAGGTTTA & \\
\hline LR-B2 & ACGTCAGGGAGAAACAATAACGAATTTTCC & \\
\hline LR-B3 & CTTAGAAACGCTGAGAAGAGTCATCCCCCT & \\
\hline LR-B4 & CAAATGCAATCAAAAATCAGGTCTGTAGCT & \\
\hline LR-B5 & CAACATGAAGTTTCATTCCATATAAATCGG & \\
\hline LR-B6 & TTGTACCGAGAAGCCTTTATTTCAACGCAA & \\
\hline LR-C1 & TTTAAAAGGATAATACATTTGAGGATTTAGAA & \\
\hline LR-C2 & TGATTGCTAAATTGCGTAGATTTTTTATTAAT & \\
\hline LR-C3 & ATTTATCAATCGTCGCTATTAATTGATTCGCC & \\
\hline LR-C4 & TGACTATTCATAAATATTCATTGAAATAGTGA & \\
\hline LR-C5 & GATTCCCAATTGCTGAATATAATGCTTTACCC & \\
\hline LR-C6 & GGATAAAACCTCAGAGCATAAAGCTAACAGTT & \\
\hline LR-D1 & CGTCAATATTTGAGTAACATTATCTAAAACAG & \\
\hline LR-D2 & AAATAAAGTTGAATACCAAGTTACAACCTTGC & \\
\hline LR-D3 & TTCTGTAAAAATCATAGGTCTGAGAATACTGC & \\
\hline LR-D4 & GGAATCGTATAGTCAGAAGCAAAGGATGGCTT & \\
\hline LR-D5 & AGAGCTTAATTCTGCGAACGAGTAAAATTAAG & \\
\hline LR-D6 & CAATAAAGATTTTTAGAACCCTCATATATTT & \\
\hline LR-E1 & GGAACAAAACTAACAACTAATAGATTAGAGC & \\
\hline LR-E2 & CGCAGAGGAAAATTATTTGCACGATTTTGC & \\
\hline LR-E3 & СTTTTTAATATATGTGAGTGAATAAAATCG & \\
\hline LR-E4 & CATCAAAAGACTGGATAGCGTCCAGACTAC & \\
\hline LR-E5 & TTTGACCAGAGGTCATTTTTGCGCGGATTG & \\
\hline LR-E6 & TAAATGCAGGCAAAGAATTAGCAGATTTAG & \\
\hline LR-F1 & TTAGGAGCGAAACCACCAGAAGGATAGAACCT & \\
\hline LR-F2 & ACCATATCCGAATTATTCATTTCAACAGTACA & \\
\hline LR-F3 & TAAATCAACCTCCGGCT & \\
\hline LR-F4 & AGTAAAATGTTTAAGATTAAGAGGAAGCCTTGCTCCT & \\
\hline LR-F6 & ACAGGCAAATGCCTGAGTAATGTGTAGGTAAA & \\
\hline LR-G1 & ATCATCATTGAGGAAGGTTATCTAAAATATCT & \\
\hline LR-G5 & ATAACCTGTAGAGAGTACCTTTAA & \\
\hline LR-G6 & GATTCAAATAGCATTAACATCCAAAATGGTCA & \\
\hline LR-H1 & AAGGAATATTCCTGATTATCAGTTTGGATT & \\
\hline LR-H2 & ATACTTCAAGATGATGAAACAAAATTTCAT & \\
\hline
\end{tabular}




\begin{tabular}{|c|c|c|}
\hline LR-H5 & TCAGGATTTTAGCTATATTTTCTTCTACTA & \\
\hline LR-H6 & ATAGTAGAGGGTGAGAAAGGCCGGAGACAG & \\
\hline LR-I2 & AAAACAAATGTCGTGCCAGCTGCATTCCAGTC & \\
\hline LR-I4 & AGCGAACCCGCCATTCAGGCTGCGCAGGCAAA & \\
\hline LR-J3 & GGGAAACCATTAATTACATTTAACACATCAAG & \\
\hline LR-J5 & GCGCCATTAGACCGGAAGCAAACTAGCTTCAA & \\
\hline LR-K1 & GTCAAAGGCCTCAAATATCAAACCCTCAATC & \\
\hline LR-K2 & ATCGGCCAAGTGTTGTTCCAGTTCTCCAAC & \\
\hline LR-K5 & AAAATAATTCTGGTGCCGGAAACCAACTGT & \\
\hline LR-K6 & TCTAGCTGCGCATTAAATTTTTGGCCATCA & \\
\hline LR-L1 & TTGCTGAAGCGAAAAACCGTCTATGCCCGAGA & \\
\hline LR-L2 & TAGGGTTGACGCGCGGGGAGAGGC & \\
\hline LR-L6 & TTAAAATTATAAATTAATGCCGGAGAGGGTAG & \\
\hline LR-M1 & TGGCCCACAAATGAAAAATCTAAAGCATCACC & \\
\hline LR-M3 & CACCCTCAGAGCCAATGAGTGAGCTAACTGGTTTGCG & \\
\hline LR-M4 & ATTACGCCATTGGCCTT & \\
\hline LR-M5 & GCTTTCATGATCGCACTCCAGCCATCTTCGCT & \\
\hline LR-M6 & CTATTTTTTAAATTGTAAACGTTATGTAGCCA & \\
\hline LR-N1 & CAGCAGCTACGTGAACCATCACTCGGCAAA & \\
\hline LR-N2 & ATCCCTTGCCAGGGTGGTTTTTAAGCCTGG & \\
\hline LR-N3 & GGTGCCTGCCACCAGAACCACCGAGGCAGG & \\
\hline LR-N4 & TCAGACGAGCTGGCGAAAGGGGTATCGGCC & \\
\hline LR-N5 & TCAGGAACAACATTAAATGTGAGTATAAGC & \\
\hline LR-N6 & AAATATTGAGAGATCTACAAAGGCTATCAG & \\
\hline LR-01 & AGTTTTTTGAAGGGAAGTGCCACGCTGAGAGC & \\
\hline LR-02 & CAGTGAGAGATGGTGGTTCCGAAACCAAATCA & \\
\hline LR-03 & CGCCGCCATCCCCGGGTACCGAGCGGTCGACT & \\
\hline LR-04 & GCAAGGCGATTAAGTTGGGTAACGGTGCATCT & \\
\hline LR-05 & CAACCCGTGAGGGGACGACGACAGGATGTGCT & \\
\hline LR-06 & $\begin{array}{l}\text { GTCATTGCCAAAAACAGGAAGATTGCGAGTAA } \\
\end{array}$ & \\
\hline LR-P1 & CGAGAAAGGGGGTCGAGGTGCCGTGGCGAAAA & \\
\hline LR-P2 & TCCTGTTTCGGGCAACAGCTGATTACACAACA & \\
\hline LR-P3 & TACGAGCCGGAAGCATAAAGTGTACTTTTCAC & \\
\hline LR-P4 & CTAGAGGAGCATTGACAGGAGGTTACCAGAGC & \\
\hline LR-P5 & GCCAGTTTCGGATTCTCCGTGGGAATAATCAG & \\
\hline LR-P6 & AAAAGCCCCTGAGAGTCTGGAGCAAACAAGA & \\
\hline LR-Q1 & TAAATCGGACGGGGAAAGCCGGCGAACGTGG & \\
\hline LR-Q2 & ACCGCCTGTGGTTTGCCCCAGCAAAAGCAC & \\
\hline LR-Q3 & CGTAATCATCCGCTCACAATTCCGCCCTTC & \\
\hline LR-Q4 & TTTCCCAGGCTTGCATGCCTGCATCGAATT & \\
\hline LR-Q5 & GCGGATTGGGGCGCATCGTAACCCCAGGGT & \\
\hline LR-Q6 & GAATCGATTATGTACCCCGGTTGACAAACG & \\
\hline LR-R1 & CCCGATTTAGAGCTTGAACCCTAAAGGGAGCC & \\
\hline LR-R2 & AGCAAGCGGTCCACGCGCCCTGAGAGAGTTGC & \\
\hline LR-R3 & CTGTGTGAAATTGTTATGGTCATAGCTGTTTC & \\
\hline
\end{tabular}




\begin{tabular}{|c|c|c|}
\hline LR-R4 & CGACGGCCAGTGCCAATCACGACGTTGTAAAA & \\
\hline LR-R5 & TCACGTTGGTGTAGATACCGTAATGGGATAGG & \\
\hline LR-R6 & ACTAGCATGTCAATCAGAACGGTAATCGTAAA & \\
\hline UD-A1 & AACTAATGCAGATACATAGGAATACCACATTC & \\
\hline UD-A2 & CAAGAACCGGATATTCTCAAGAGTAATCTTGA & \\
\hline UD-A3 & GAAGGCACCAACCTAATACGTAATGCCACTAC & \\
\hline UD-A4 & TTAATTGTATCGGTTTGCTCCAAAAGGAGCCT & \\
\hline UD-A5 & TAGCAAGCCCAATAGGCCCTCATTTTCAGGGA & \\
\hline UD-A6 & CTTGAGTAACAGTGCCTTAACGGGGTCAGTGC & \\
\hline UD-B1 & AAAAGGAATTACGAGGCATAGTAATTACAGGT & \\
\hline UD-B2 & AAATCAACTCATCAGTTGAGATTTAACGCC & \\
\hline UD-B3 & GAGGCAAAGCTGGCTGACCTTCAATTACCC & \\
\hline UD-B4 & TGCTTTCGATTAAACGGGTAAAAAACGAAA & \\
\hline UD-B5 & GTACCGTAATCTCCAAAAAAAAGATCAGCT & \\
\hline UD-B6 & ACAGTTAACCCTCAGAGCCACCAAACCCAT & \\
\hline UD-C1 & AGAAAGATGTAACAAAGCTGCTCACAGACCAG & \\
\hline UD-C2 & GCGCATAGAGAATACACTAAAACATCATGAGG & \\
\hline UD-C3 & AAGTTTCCAGGTGAATTTCTTAAATTTTTTTCA & \\
\hline UD-C4 & CGTTGAAAACACTGAGTTTCGTCAACCCTCAG & \\
\hline UD-C5 & AACCGCCATGCCCCCTGCCTATTTTGATGATA & \\
\hline UD-C6 & CAGGAGTGTACTGGTAATAAGTTCGTATAA & \\
\hline UD-D1 & CTATCATAACCCTCGTTTACCAGGTTAATAA & \\
\hline UD-D2 & ATAAGGCTTAACGGAACAACATTAGAGCAACA & \\
\hline UD-D3 & TGACCCCCACAGATGAACGGTGTATTCAGTGA & \\
\hline UD-D4 & TACCGATAAGGACTAAAGACTTTTCTCATCTT & \\
\hline UD-D5 & AACTACAAGAATTGCGAATAATAACAGCTTGA & \\
\hline UD-D6 & ATTATTCTCACCCTCAGAACCGCCCCAGTACA & \\
\hline UD-E1 & AACGAACTGCCCTGACGAGAAACAACTTTG & \\
\hline UD-E2 & AAAGAGGAGCGATTATACCAAGGCTACAGA & \\
\hline UD-E3 & GGCTTTGGTTGCGCCGACAATGAAGGAACA & \\
\hline UD-E4 & ACTAAAGCGCCTGTAGCATTCCGAGGTTTA & \\
\hline UD-E5 & GTACCGCGAAACATGAAAGTATTACCGTTC & \\
\hline UD-E6 & CAGTAAGCGTCATACATGGCTTTCGGAACCT & \\
\hline UD-F1 & AAAAACCAAAATAGCGAGAGGCTTTACCAGTC & \\
\hline UD-F2 & CGAGTAGTGGGAAGAAAAATCTACACGACGAT & \\
\hline UD-F3 & AAAGTACAAGGGAACCGAACTGACCACCAGAA & \\
\hline UD-F5 & CCCTCATAGCGGAGTGAGAATAGAACAACAA & \\
\hline UD-F6 & TGAGACTCGTATCACCGTACTCAGACAGACAG & \\
\hline UD-G1 & AGGACGTTAAATTGGGCTTGAGATCAGACGGT & \\
\hline UD-G4 & AGTTTCAGTTAGCGTAACGATCTATAGCCCG & \\
\hline UD-G6 & ATGGAAAGCGCAGTCTCTGAATTTAAGAGGC & \\
\hline UD-H1 & AAGTTTTGCCAGAGGGGGTATTTTTTTTTTTGGGTTATA & \\
\hline $\mathrm{UD}-\mathrm{H} 3$ & ATAAATTGAGCCGGAACGAGGCGGGTTTAA & \\
\hline UD-H4 & GCTGAGGCCGTCACCCTCAGCAGTCGCCTG & \\
\hline UD-H5 & TGTCGTCTGGATTTTGCTAAACATTCGGTC & \\
\hline
\end{tabular}




\begin{tabular}{|c|c|c|}
\hline UD-H6 & CGGGGTTTAGGGTTGATATAAGTAAAGTTT & \\
\hline UD-HI6 & AATAAATCCTCATTGGATTAG & \\
\hline UD-I2 & TGTTACTTTGTCGAAATCCGCGACGAACAAGC & \\
\hline UD-I3 & TGCGGGATTTGCAGGGAGTTAAAGGAAACGAT & \\
\hline UD-I4 & TCTGTATGTTCCAGACGTTAGTAACGCAATAA & \\
\hline UD-J3 & AAGCCGTTGTACCGCACTCATCGACTGCTCCA & \\
\hline UD-J4 & TTTTTGTTATCCCAATCCAAATAAGCCGCTTT & \\
\hline UD-J5 & TAACGGAAAAGGAAACCGAGGAAAATGAATTT & \\
\hline UD-JK1 & TAACTATATGTAAATCAACAGT & \\
\hline UD-K1 & AGGGCTTATGTAATTTAGGCAGCGGGTATT & \\
\hline UD-K2 & AAACCAATTTATTTTCATCGTAACAGCCAT & \\
\hline UD-K3 & ATTATTTTAACGTCAAAAATGAGAACAAAG & \\
\hline UD-K4 & TTACCAGTACCCAAAAGAACTGGGTGAATT & \\
\hline UD-K6 & GAACCGCCACCCTCAGAGCCTTTTTTTTTTTCACAAACA & \\
\hline UD-L1 & AAATCCAATCGCAAGACAAAGAATATACAAA & \\
\hline UD-L3 & ACCGCGCCTTATCATTCCAAGAAAGGCATTT & \\
\hline UD-L6 & TACCATTAGAAATTATTCATTAAAGCATGATT & \\
\hline UD-M1 & TTCTTACCGTAATAAGAGAATATATAATCGGC & \\
\hline UD-M2 & TGTCTTTCCCAATAGCAAGCAAATTTCCAGA & \\
\hline UD-M4 & TTTAAGAATTATTACGCAGTATGTAAGGTAAA & \\
\hline UD-M5 & TATTGACGGCAAGGCCGGAAACGTAGCCACCA & \\
\hline UD-M6 & CCGGAACCGCCTCCCTCAGAGCCGAGCACCAT & \\
\hline UD-N1 & AAACTTTTTCAAATATATTTTAGTCTAGAAAA & \\
\hline UD-N2 & GACAAAAGTAGTATCATATGCGTCGCGAGA & \\
\hline UD-N3 & AGAAGGCTGTAGAAACCAATCAAAAGTACC & \\
\hline UD-N4 & GAAGCGCACGCTAACGAGCGTCTCAGATAT & \\
\hline UD-N5 & CGTAGAAAATAGCTATCTTACCGAAACAGG & \\
\hline UD-N6 & GAAACCATCCGATTGAGGGAGGGTAGCAAA & \\
\hline UD-01 & AGCCTGTTGTAAAGTAATTCTGTCCCTAATTT & \\
\hline UD-02 & ACGAGCATTATCCGGTATTCTAAGTCCTGAAT & \\
\hline UD-03 & CTTACCAATTAGACGGGAGAATTAAAACAATG & \\
\hline UD-04 & AAATAGCAATACATACATAAAGGTAAAGGGCG & \\
\hline UD-05 & ACATTCAACGATAGCAGCACCGTAATCTTTTC & \\
\hline UD-06 & ATAATCAAAATCACCGGAACCAGCACCAAT & \\
\hline UD-P1 & TCTTCTGACCTAAATTTAATGGTTAAATAAG & \\
\hline UD-P2 & GACAATAAACCGGAATCATAATTATAATTTCA & \\
\hline UD-P3 & GCGTTTTAAAAAATAATATCCCATCAGACGAC & \\
\hline UD-P4 & CCCTGAACCCCAGCTACAATTTTAAACGCGAG & \\
\hline UD-P5 & ATAAAAGACAATAATAAGAGCAAGACTGAACA & \\
\hline UD-P6 & CGACAGAAACCAGCGCCAAAGACAGGCAACAT & \\
\hline UD-Q1 & AATAAACACAACATGTTCAGCTTAAGTCCT & \\
\hline UD-Q2 & GAACAAGGCGAACCTCCCGACTAGTTGCTA & \\
\hline UD-Q3 & TTTTGCAAAAGTCAGAGGGTAAGAATTGAG & \\
\hline UD-Q4 & TTAAGCCAACGCAAAGACACCAAAATTCAT & \\
\hline UD-Q5 & ATGGTTTTCAAGTTTGCCTTTATTCGGTCA & \\
\hline
\end{tabular}




\begin{tabular}{|c|c|c|}
\hline UD-Q6 & TAGCCCCCTTATTAGCGTTTGCCATCAGTAG & \\
\hline UD-R1 & GTGATAAATAAGGCGTTTGAAATACCGACCGT & \\
\hline UD-R2 & $\begin{array}{l}\text { GTTTATCAACAATAGAAATGCAGAACGCGCCT } \\
\end{array}$ & \\
\hline UD-R3 & GCCTTAAATCAAGATTTGCGGGAGGTTTTGAA & \\
\hline UD-R4 & GAGAGATAACCCACAATTGAGCGCTAATATCA & \\
\hline UD-R5 & TGTCACAATCAATAGACGGAATAAGTTTATTT & \\
\hline UD-R6 & CGTTTTCATCGGCATTGCGTCAGACTGTAGCG & \\
\hline$c X-1 a$ & TTTGATAATTAGATACATTTCTTTCAAAAG & \\
\hline$c X-1 b$ & TTTCAACTAGAACTGGCTCATTATTGTTTGCATAAATCAT & \\
\hline$c X-2 a$ & $\begin{array}{l}\text { TCGAGCCAAGTATAAAGCCAACGCTGCTTTGGTGCGGAATT } \\
\end{array}$ & \\
\hline$c X-2 b$ & AGCAAAAGTGAATAATGGAAGTTTTGATGC & \\
\hline$c x-3 a$ & CGAAAGACTTTTATTAATCATTGTGAATTATTTAAC & \\
\hline$c X-3 b$ & GCGATTTTTTCAAATATCGCGTTTTAATTCGCCAACAGG & \\
\hline$C X-4 a$ & AACGCCTTTTTTTTAATGGAAATTACCTG & \\
\hline$c X-4 b$ & TTGAATTACCTTAACAATTGAGAATCGCCATACCTTAT & \\
\hline$C X-5 a$ & CAATCATAACGGAGATTTCAAATTTGGGG & \\
\hline$c X-5 b$ & GCTCATTTGAAAAGGTGGCATTTTTGTATCACGAAAGAC & \\
\hline$c X-6 a$ & $\begin{array}{l}\text { GCCTTTACGCCAGTTACAAAATAAGGATTTTGATGATGGC } \\
\end{array}$ & \\
\hline$c X-6 b$ & GAGTCCACAATATAATCCTGATTATCATT & \\
\hline$c X-7 a$ & GCCTAATTAGTTGGCAAATCAACAGTTGA & \\
\hline$c X-7 b$ & AATATCTGGTCTTTTTAAGAGAGAATAACATAAAAGCCCTT & \\
\hline$C X-8 a$ & TCAAATCACCATTCCCAACGAGGGTAGCAACGCGCGAAAC & \\
\hline$C X-8 b$ & CCATCGTTTCAATATGATATTCAACCGT & \\
\hline$c X-9 a$ & CGCGAGCTTTTAACCAATAGGTTTTCAAC & \\
\hline$c X-9 b$ & AGCATCGGACGCATAACCGATATAACTTTAACTTAAATCA & \\
\hline CX-10a & AATTCATCTATTAAAGAACGTTTAGATAGCCAAATAGCA & \\
\hline$c X-10 b$ & AAGACTCCAAGTAAGCTTGGATGGAACAA & \\
\hline$c X-11 a$ & $\begin{array}{l}\text { TGGGAAGGGCGTTGAGTGCTCAGTACCAGGCGTTTGGGA } \\
\end{array}$ & \\
\hline$c X-11 b$ & CCGTCTTATCGGTGCGGGCCGCTTTCCG & \\
\hline$C X-12 a$ & ATTAGATTTTGCGTTGCGCTCACTGCCCGCTTTAATGA & \\
\hline$c X-12 b$ & CACATTAATTGCCTCACCGACTTGAGCCAGATAAGTG & \\
\hline$c X-13 a$ & GCACCGCTTCGCGTCTGGCCTTTTTGCCAGA & \\
\hline$c X-13 b$ & GAATAGGTCTCAAGAGAAGGATTAAAATTTTCCATATTTTG & \\
\hline CX-14a & ATCACCGAGCAAAATCACCAGTCCATTTATACAGGGCGA & \\
\hline$C X-14 b$ & TATTGGGCATAAATCAAAAGATTTCCCTCA & \\
\hline
\end{tabular}


Table S2: List of internal docking staples for DNA origami cross-tile.

\begin{tabular}{|l|l|l|}
\hline Name & Sequence & Notes \\
\hline LR-I2_M2-8 & AAAACAAATGTCGTGCCAGCTGCATTCCAGTCTTACTTGTGA & $8 \mathrm{nt}$ M2 dock \\
\hline LR-J5_M2-8 & GCGCCATTAGACCGGAAGCAAACTAGCTTCAATTACTTGTGA & $8 \mathrm{nt}$ M2 dock \\
\hline LR-I4_M2-8 & AGCGAACCCGCCATTCAGGCTGCGCAGGCAAATTACTTGTGA & $8 \mathrm{nt}$ M2 dock \\
\hline LR-J3_M2-8 & GGGAAACCATTAATTACATTTAACACATCAAGTTACTTGTGA & $8 \mathrm{nt}$ M2 dock \\
\hline UD-I2_M2-8 & CTCATCGACTGCTCCATGTTACTTTGTCGAAATTACTTGTGA & $8 \mathrm{nt}$ M2 dock \\
\hline UD-I3_M2-8 & CCAAATAAGCCGCTTTTGCGGGTTTGCAGGGTTACTTGTGA & $8 \mathrm{nt}$ M2 dock \\
\hline UD-J4_M2-8 & AGTTAAAGGAAACGATTTTTTGTTATCCCAATTTACTTGTGA & $8 \mathrm{nt}$ M2 dock \\
\hline UD-J5_M2-8 & GTTAGTAACGCAATAATAACGGAAAAGGAACTTACTTGTGA & $8 \mathrm{nt}$ M2 dock \\
\hline UD-I4 & CGAGGAAAATGAATTTTCTGTATGTTCCAGAC & \\
\hline UD-J3 & TCCGCGACGAACAAGCAAGCCGTTGTACCGCA & \\
\hline
\end{tabular}

Table S3: List of edge staples for DNA origami cross-tile.

\begin{tabular}{|c|c|c|}
\hline Name & Sequence & Notes \\
\hline P-U1 & GTGTCGTAGACACGTGATAAATAAGGCGTTTGAAATACCGACCGTGTGTCGTAGACAC & Hairpin \\
\hline$P-U 2$ & GTGTCGTAGACACGTTTATCAACAATAGAAATGCAGAACGCGCCTGTGTCGTAGACAC & Hairpin \\
\hline$P-U 3$ & GTGTCGTAGACACGCCTTAAATCAAGATTTGCGGGAGGTTTTGAAGTGTCGTAGACAC & Hairpin \\
\hline$P-U 4$ & GTGTCGTAGACACGAGAGATAACCCACAATTGAGCGCTAATATCAGTGTCGTAGACAC & Hairpin \\
\hline P-U5 & GTGTCGTAGACACTGTCACAATCAATAGACGGAATAAGTTTATTTGTGTCGTAGACAC & Hairpin \\
\hline P-D2 & GTGTCGTAGACACCAAGAACCGGATATTCTCAAGAGTAATCTTGAGTGTCGTAGACAC & Hairpin \\
\hline P-D3 & GTGTCGTAGACACGAAGGCACCAACCTAATACGTAATGCCACTACGTGTCGTAGACAC & Hairpin \\
\hline P-D4 & GTGTCGTAGACACTTAATTGTATCGGTTTGCTCCAAAAGGAGCCTGTGTCGTAGACAC & Hairpin \\
\hline P-D5 & GTGTCGTAGACACTAGCAAGCCCAATAGGCCCTCATTTTCAGGGAGTGTCGTAGACAC & Hairpin \\
\hline P-D6 & GTGTCGTAGACACCTTGAGTAACAGTGCCTTAACGGGGTCAGTGCGTGTCGTAGACAC & Hairpin \\
\hline$P-L 2$ & GTGTCGTAGACACAGTACCTTTTACATCGATGAATATACAGTAACGTGTCGTAGACAC & Hairpin \\
\hline$P-L 3$ & GTGTCGTAGACACGATAGCTTAGATTAAGTCCTTGAAAACATAGCGTGTCGTAGACAC & Hairpin \\
\hline $\mathrm{P}-\mathrm{L} 4$ & GTGTCGTAGACACAACGAGAATGACCATATTTAAACAGTTCAGAAGTGTCGTAGACAC & Hairpin \\
\hline$P-L 5$ & GTGTCGTAGACACAAAGTACGGTGTCTGGTTTTAAATATGCAACTGTGTCGTAGACAC & Hairpin \\
\hline P-L6 & GTGTCGTAGACACTGTAATACTTTTGCGGAAAAACATTATGACCCGTGTCGTAGACAC & Hairpin \\
\hline $\mathrm{P}-\mathrm{R} 1$ & GTGTCGTAGACACCCCGATTTAGAGCTTGAACCCTAAAGGGAGCCGTGTCGTAGACAC & Hairpin \\
\hline$P-R 2$ & GTGTCGTAGACACAGCAAGCGGTCCACGCGCCCTGAGAGAGTTGCGTGTCGTAGACAC & Hairpin \\
\hline $\mathrm{P}-\mathrm{R3}$ & GTGTCGTAGACACCTGTGTGAAATTGTTATGGTCATAGCTGTTTCGTGTCGTAGACAC & Hairpin \\
\hline P-R4 & GTGTCGTAGACACCGACGGCCAGTGCCAATCACGACGTTGTAAAAGTGTCGTAGACAC & Hairpin \\
\hline P-R5 & GTGTCGTAGACACTCACGTTGGTGTAGATACCGTAATGGGATAGGGTGTCGTAGACAC & Hairpin \\
\hline P-U5_M1-7 & GTGTCGTAGACACTGTCACAATCAATAGACGGAATAAGTTTATTTGTATACATCA & 7 nt M1 dock \\
\hline P-U6_M1-7 & GTGTCGTAGACACCGTTTTCATCGGCATTGCGTCAGACTGTAGCGGTATACATCA & 7 nt M1 dock \\
\hline P-U6_M1-8 & $\begin{array}{l}\text { GTGTCGTAGACACCGTTTTCATCGGCATTGCGTCAGACTGTAGCGTTATACATCT } \\
\end{array}$ & 8 nt M1 dock \\
\hline P-D1_M1-7 & GTGTCGTAGACACAACTAATGCAGATACATAGGAATACCACATTCGTATACATCA & 7 nt M1 dock \\
\hline P-D1_M1-8 & GTGTCGTAGACACAACTAATGCAGATACATAGGAATACCACATTCAATACATCT & 8 nt M1 dock \\
\hline P-D2_M1-7 & GTGTCGTAGACACCAAGAACCGGATATTCTCAAGAGTAATCTTGAGTATACATCA & $7 \mathrm{nt}$ M1 dock \\
\hline P-L1_M1-7 & GTGTCGTAGACACGACAACTCGTATTAAAACTTTACAAACAATTCGTATACATCA & 7 nt M1 dock \\
\hline P-L1_M1-8 & GTGTCGTAGACACGACAACTCGTATTAAAACTTTACAAACAATTCTTATACATCT & 8 nt M1 dock \\
\hline P-L2_M1-7 & GTGTCGTAGACACAGTACCTTTTACATCGATGAATATACAGTAACGTATACATCA & 7 nt M1 dock \\
\hline
\end{tabular}




\begin{tabular}{|l|l|l|}
\hline P-R5_M1-7 & GTGTCGTAGACACTCACGTTGGTGTAGATACCGTAATGGGATAGGGTATACATCA & 7 nt M1 dock \\
\hline P-R6_M1-7 & GTGTCGTAGACACACTAGCATGTCAATCAGAACGGTAATCGTAAAGTATACATCA & 7 nt M1 dock \\
\hline P-R6_M1-8 & GTGTCGTAGACACACTAGCATGTCAATCAGAACGGTAATCGTAAATATACATCT & 8 nt M1 dock \\
\hline
\end{tabular}

Table S4: List of modified oligomers for imaging and filtration.

\begin{tabular}{|l|l|l|}
\hline Name & Sequence & Notes \\
\hline M1' & CTAGATGTAT/Cy3b/ & Imager for dock M1 \\
\hline M2' & ACTCACAAGT/Cy3b/ & Imager for dock M2 \\
\hline Ac-5T-M1' -7 & /5Acryd/TTTTTAGATGTAT & $\begin{array}{l}\text { Acrydite-labelled, } \\
7 \text { nt M1' }\end{array}$ \\
\hline 47pT-M1' -8 & TTTTTTTTTTTTTTTTTTTTTTTTTTTTTTTTTTTTTTTTTTTTTTTAGATGTAT & $\begin{array}{l}\text { Duplexing strand, } \\
8 \text { nt M1' }\end{array}$ \\
\hline
\end{tabular}

Table S5: Thermocycler recipe for DNA origami synthesis.

\begin{tabular}{|c|c|c|c|c|}
\hline Step & Initial temp. & Cycles & $\Delta \mathbf{T} /$ Cycle & Time / Cycle \\
\hline$\#$ & $\left({ }^{\circ} \mathrm{C}\right)$ & $\#$ & $\left({ }^{\circ} \mathrm{C}\right)$ & 15 \\
\hline 1 & 70 & 1 & 0 & 0.75 \\
\hline 2 & 70 & 50 & -0.1 & 0.75 \\
\hline 3 & 65 & 50 & -0.1 & 0.75 \\
\hline 4 & 60 & 50 & -0.1 & 2 \\
\hline 5 & 55 & 50 & -0.1 & 2 \\
\hline 6 & 50 & 50 & -0.1 & 2 \\
\hline 7 & 45 & 50 & -0.1 & 1.5 \\
\hline 8 & 40 & 50 & -0.1 & 1.5 \\
\hline 9 & 35 & 50 & -0.5 & 0.5 \\
\hline 10 & 30 & 20 & 0 & Hold \\
\hline
\end{tabular}




\section{References}

1. Ovesny, M. Computational Methods in Single Molecule Localization Microscopy. Doctor of Philosophy, Charles University, Prague, 2016.

2. Schneider, C. A.; Rasband, W. S.; Eliceiri, K. W., NIH Image to ImageJ: 25 Years of Image Analysis. Nat. Methods 2012, 9, 671-675.

3. Nečas, D.; Klapetek, P., Gwyddion: An Open-Source Software for SPM Data Analysis. Open Physics 2012, 10 (1), 181-188.

4. Rehman, F. N.; Audeh, M.; Abrams, E. S.; Hammond, P. W.; Kenney, M.; Boles, T. C., Immobilization of Acrylamide-Modified Oligonucleotides by Co-Polymerization. Nucleic Acids Res. 1999, 27 (2), 649-655.

5. Blumhardt, P.; Stein, J.; Mucksch, J.; Stehr, F.; Bauer, J.; Jungmann, R.; Schwille, P., Photo-Induced Depletion of Binding Sites in DNA-PAINT Microscopy. Molecules 2018, 23 (12), 3165.

6. Scheible, M. B.; Pardatscher, G.; Kuzyk, A.; Simmel, F. C., Single Molecule Characterization of DNA Binding and Strand Displacement Reactions on Lithographic DNA Origami Microarrays. Nano Lett. 2014, 14 (3), 1627-1633.

7. $\quad$ Douglas, S. M.; Marblestone, A. H.; Teerapittayanon, S.; Vazquez, A.; Church, G. M.; Shih, W. M., Rapid Prototyping of 3D DNA-Origami Shapes with Cadnano. Nucleic Acids Res. 2009, 37 (15), 5001-5006.

8. Liu, W.; Zhong, H.; Wang, R.; Seeman, N. C., Crystalline Two-Dimensional DNA-Origami Arrays. Angew. Chem. Int. Ed. 2011, 50 (1), 264-267.

9. Aghebat Rafat, A.; Pirzer, T.; Scheible, M. B.; Kostina, A.; Simmel, F. C., Surface-Assisted Large-Scale Ordering of DNA Origami Tiles. Angew. Chem. Int. Ed. 2014, 53 (29), 7665-7668.

10. Tikhomirov, G.; Petersen, P.; Qian, L., Fractal Assembly of Micrometre-Scale DNA Origami Arrays with Arbitrary Patterns. Nature 2017, 552 (7683), 67-71.

11. Aebi, U.; Pollard, T. D., A Glow Discharge Unit to Render Electron Microscope Grids and Other Surfaces Hydrophilic. J. Electron Microsc. Tech. 1987, 7 (1), 29-33. 